
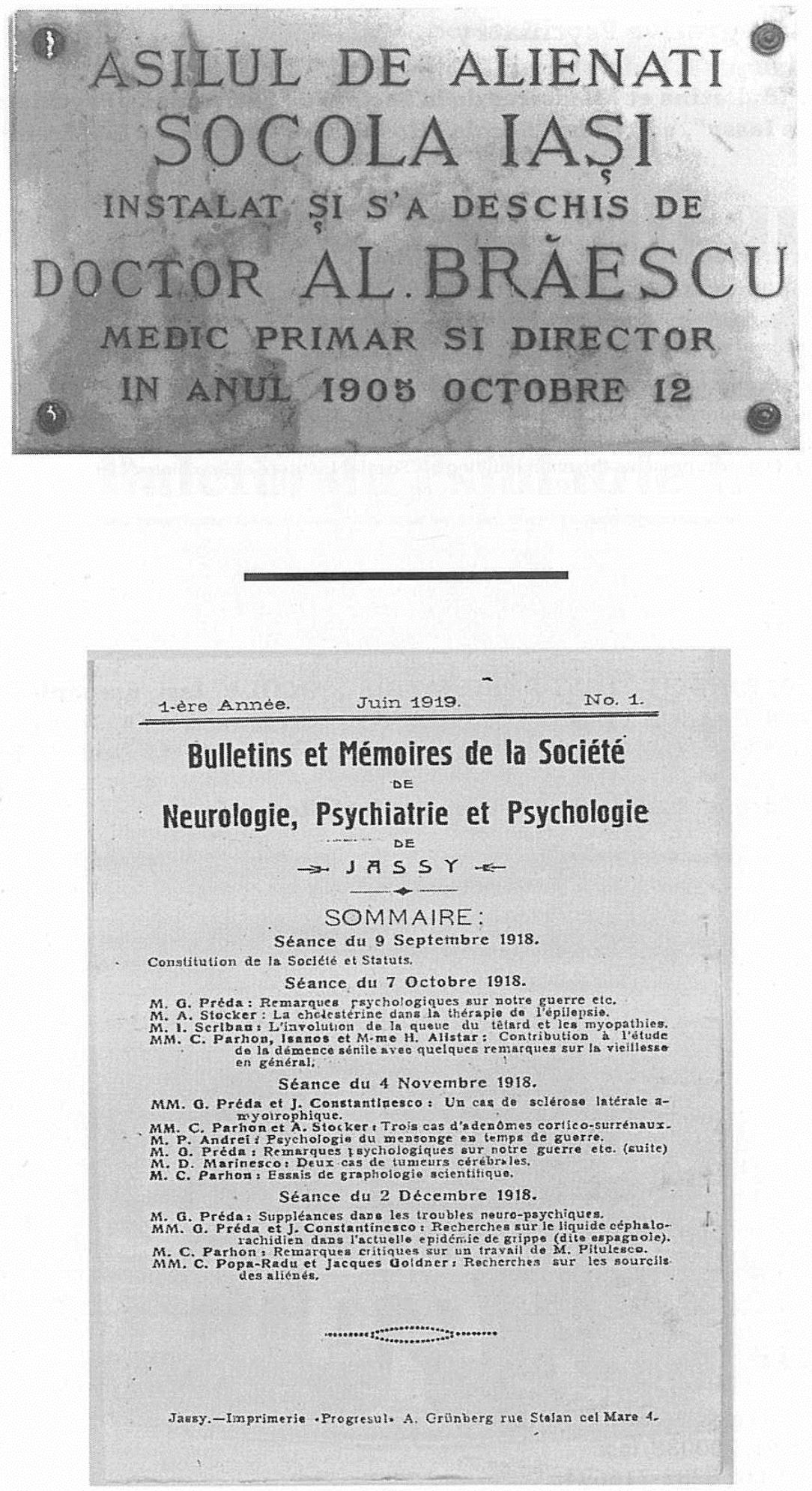


\section{Bulletin of Integrative Psychiatry}

New series of "Bulletins et Mémoires de la Société de Neurologie, Psychiatrie et Psychologie de Iassy", edited by "Socola" Hospital of Psychiatry Iaşi from 1919 to 1946

Editorial Board: "Socola" Institute of Psychiatry Iaşi

Address: Şoseaua Bucium nr. 36, cod poştal 700282, Iaşi, România

Images on the first page represent: Inaugural board of "Socola" Hospital of Psychiatry Iaşi The cover of the first number of "Bulletins et Mémoires de la Société de Neurologie, Psychiatrie et Psychologie de Iassy"

The image on the first cover represents the main building of "Socola" Institute of Psychiatry Iasi

ISSN: 1453-7257

\section{Copyright @ 2017 INSTITUTUL DE PSIHIATRIE, „SOCOLA" Iaşi, România}

The papers published in "The Bulletin of Integrative Psychiatry" are protected by copyright. Their full or partial publication in other journal is allowed only with the written approval of "SOCOLA" Institute of Psychiatry Iaşi, Romania.

Published by "Socola" Institute of Psychiatry Iaşi, Romania

\section{"Gr.T. Popa" Pullisher}

University of Medicine and Pharmacy Iaşi

$16^{\text {th }}$ Universităţii Str.

All rights of this work are reserved to the authors and „Gr.T. Popa" Publisher Iaşi. No part of this publication may be reproduced or transmitted by any means, electronical or mechanical, including photocopying without written permission from the authors or publisher. 
\begin{tabular}{|l|l} 
Seria Nouă & New Series
\end{tabular}

An XXVI nr. 3 (86) Year XXVI No. 3 (86)

Septembrie 2020 September 2020

Frecvența: Trimestrială Frequency: Quarterly

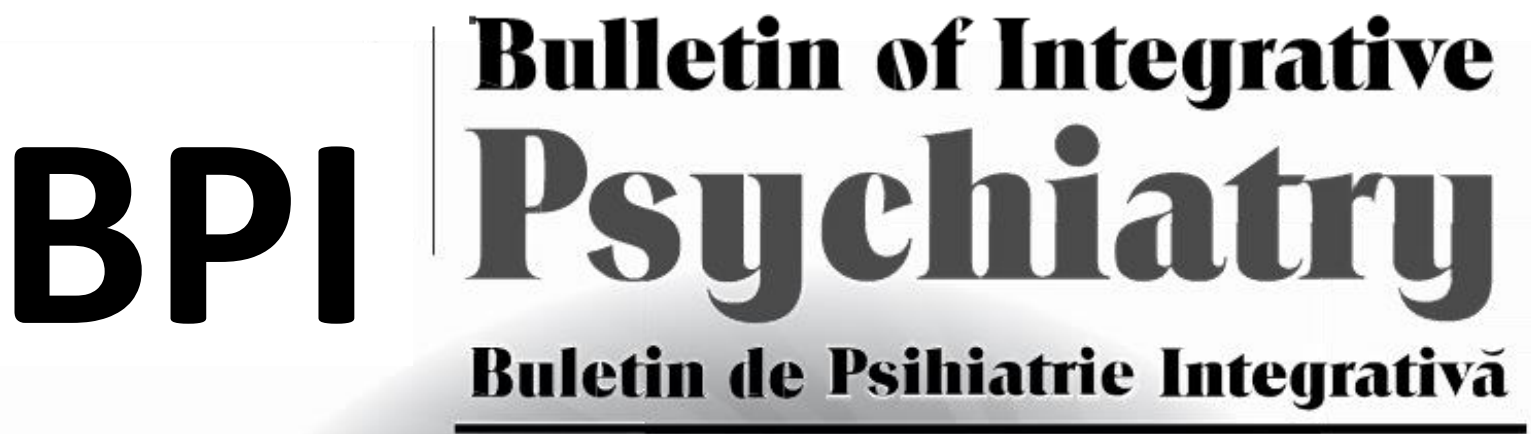

Buletin de Psihiatrie Integrativă

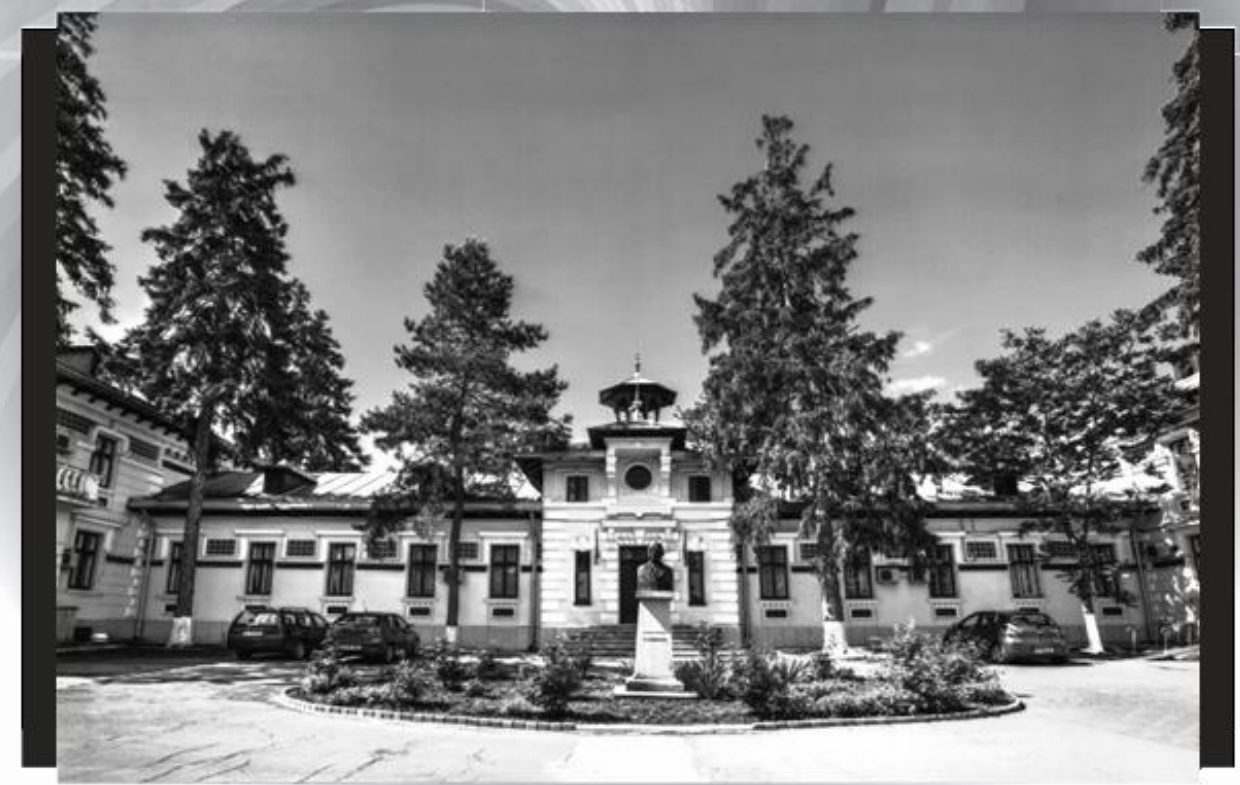

Official Publication of

"SOCOLA" INSTITUTE OF PSYCHIATRY

Iași, Romania 


\section{PUBLISHER: "SOCOLA" INSTITUTE OF PSYCHIATRY IAȘI}

\section{EDITORIAL BOARD}

Chief Editor:

Professor Roxana Chiriţă-MD, PhD

Grigore T. Popa University of Medicine and

Pharmacy, Iasi, Romania

\section{Scientific Editor:}

Professor Vasile Chiriță-MD, PhD

Honorary Member of the Romanian Academy of Medical Science

Managing Editors:

Alexandra Boloş-MD, PhD

Grigore T. Popa University of Medicine and

Pharmacy, Iasi, Romania

Romeo P. Dobrin-MD, PhD

Grigore T. Popa University of Medicine and

Pharmacy, Iasi, Romania

Deputy editors:

Prof. Cristinel Ştefănescu-MD, PhD

Grigore T. Popa University of Medicine and

Pharmacy, Iasi, Romania

Prof. Călina Gogălniceanu

Al. I.Cuza University, Faculty of Letters, Iasi,

Romania

Laura Pricop-researcher, PhD

Al. I. Cuza University -Interdisciplinary Research in Social-Human Sciences Iasi, Romania

Alin Ciobîcă-researcher, $\mathbf{P h D}$

Al. I. Cuza University Iasi, Romania

Editors:

Radu Andrei-MD, PhD

Socola Institute of Psychiatry, Iasi, Romania

Ovidiu Eugen Alexinschi-MD, PhD

Socola Institute of Psychiatry, Iasi, Romania

Adrian Dumitru Cantemir- MD, PhD

Grigore T. Popa University of Medicine and

Pharmacy, Iasi, Romania

Gabriela Elena Chele-MD, PhD

Grigore T. Popa University of Medicine and

Pharmacy, Iasi, Romania

Oana Crețu-MD, PhD

Socola Institute of Psychiatry, Iasi, Romania

Ana Caterina Cristofor-MD, PhD

Grigore T. Popa University of Medicine and

Pharmacy, Iasi, Romania

Irina Dobrin-MD, PhD

Grigore T. Popa University of Medicine and

Pharmacy, Iasi, Romania
Tudor Florea-MD

Grigore T. Popa University of Medicine and

Pharmacy, Iasi, Romania

Anton Knieling-MD, PhD

Grigore T. Popa University of Medicine and Pharmacy, Iasi, Romania

Ioan Mihalache-MD, PhD

Socola Institute of Psychiatry, Iasi, Romania

Florin Mitu-MD, PhD

Grigore T. Popa University of Medicine and Pharmacy, Iasi, Romania

Petronela Nechita-MD, PhD

Socola Institute of Psychiatry, Iasi, Romania

Matei Palimariciuc-MD

Grigore T. Popa University of Medicine and

Pharmacy, Iasi, Romania

Elena Popescu-MD

Grigore T. Popa University of Medicine and

Pharmacy, Iasi, Romania

Raluca Ioana Prepeliță-MD

Socola Institute of Psychiatry, Iasi, Romania Dania Andreea Radu-MD

Grigore T. Popa University of Medicine and Pharmacy, Iasi, Romania

Irina Săcuiu-MD, PhD

Socola Institute of Psychiatry, Iasi, Romania

Laura Gheucă Solovăstru-MD, PhD

Grigore T. Popa University of Medicine and Pharmacy, Iasi, Romania

Andreea Silvana Szalontay-MD, PhD

Grigore T. Popa University of Medicine and Pharmacy, Iasi, Romania

Serban Turliuc-MD, PhD

Grigore T. Popa University of Medicine and Pharmacy, Iasi, Romania

Gabriela Ștefănescu- MD, PhD

Grigore T. Popa University of Medicine and

Pharmacy, Iasi, Romania

Ilinca Untu-MD

Grigore T. Popa University of Medicine and Pharmacy, Iasi, Romania

\section{Secretaries}

Vlad Iacob-MD

Socola Institute of Psychiatry, Iasi, Romania

Bianca Georgiana Secrieru -MD

Socola Institute of Psychiatry, Iasi, Romania 


\section{PUBLISHER: "SOCOLA" INSTITUTE OF PSYCHIATRY IAȘI}

\author{
ADVISORY BOARD: \\ ALLILAIRE J.F. (France) \\ ANDREI ELENA MIHAELA (Romania) \\ ASTĂRĂSTOAE VASILE (Romania) \\ BĂLĂCEANU-STOLNICI CONSTANTIN \\ (Romania) \\ BILD WATER (Romania) \\ BURLEA(CIUBARĂ) ANA-MARIA (Romania) \\ BURLUI VASILE (Romania) \\ CÂȘLARU DIANA (Romania) \\ COSMAN DOINA (Romania) \\ COSMOVICI NICOLAE (Romania) \\ COŞCIUG ION (Republic of Moldavia) \\ CĂRUNTU IRINA (Romania) \\ DAFINOIU ION (Romania) \\ DAVIDSON MICHAEL (Israel) \\ DEHELEAN LIANA (Romania) \\ DI FIORINO MARIO (Italy) \\ ENĂTESCU VIRGIL (Romania) \\ ENĂTESCU VIRGIL RADU (Romania) \\ FODOREANU LIANA (Romania) \\ FORNA NORINA CONSUELA (Romania) \\ GABOŞ-GRECU IOSIF (Romania) \\ GEORGESCU ȘTEFAN OCTAVIAN (Romania) \\ GOGĂLNICEANU PETRUŢ (UK) \\ HABOT SIMON (Israel)
}

HULUBAŞ ADINA (Romania)

KAMPITS PETER (Austria)

KNIGHT BERNARD (USA)

LADEA MARIA (Romania)

LAZARESCU MIRCEA (Romania)

LEPPEE MARCEL (Croatia)

MANEA MIRELA (Romania)

MARINESCU DRAGOS (Romania)

MICLUŢIA IOANA (Romania)

NACU ANATOL (Republic of Moldavia)

NIREŞTEAN AUREL (Romania)

NISHARA BRIAN (Canada)

OPREA VALENTIN (Republic of Moldavia)

PRELIPCEANU DAN (Romania)

PIANI FRANCESCO (Italy)

PRUS ELENA (Republic of Moldavia)

REVENCO MIRCEA (Republic of Moldavia)

RUJESCU DAN (Germany)

SCRIPCARIU VIOREL (Romania)

SCRIPCARU CĂLIN (Romania)

STOCKMAN RENE (Belgium)

ȘERBAN IONELA LĂCRĂMIOARA (Romania)

TĂRCOVEANU EUGEN (Romania)

TIMOFTE DANIEL (Romania)

TUDOSE CĂTĂLINA (Romania)

NUNO VIEIRA DUARDE (Portugal)

YOUNG ALLAN (UK) 

The Bulletin of Integrative Psychiatry tries to continue the tradition initiated at "Socola" Hospital in 1919, when a group of intellectuals, medical doctors and personalities from other professions founded the Society of Neurology, Psychiatry and Psychology in Iaşi. Even from its beginnings, the Society edited a journal entitled "Bulletins et Mémoires de la Société de Neurologie, Psychiatrie et Psychologie de Iassy", the first publication of the kind in Romania, which was unique also by its vision and opening towards biology, psychology, sociology and philosophy and by its prestigious board of editors: C. I. Parhon, Gh. Preda, Constantin Fedeleș, Arnold Stocker, P. Andrei, Corneliu Popa-Radu, I. A. Scriban, well known personalities, some of them being physicians of great culture and scientific qualification.

Starting from 1920, the Association and its Bulletin, born and edited at "Socola", due to their remarkable scientific activity have contributed to the organization of 18 congresses, which are mentioned in the description of "Socola" Hospital activities.

In 1947, the last number of "The Bulletin of the Society", edited in French, was banned as a result of the interdictions imposed by extremist tendencies. From its first number in 1919 and until 1947, "The Bulletin of the Society" published 2,412 articles.

The journal or "The Bulletin of the Society" has appeared under several titles: "Bulletin et Mémoires de la Société de Neurologie, Psychiatrie et Psychologie de Iassy" (between 1919 and 1922), then "Bulletin de l'Association des Psychiatres Roumains" and from 1923 it has changed its title several times.

After the year 1947, all publications at "Socola" Hospital were included in the "Medico-Surgical Journal of the Society of Physicians and Naturalists in Iaşi", another prestigious scientific journal which has been published without interruption since 1886.

Starting from 1994, Professor Dr. Tadeusz Pirozynski, Professor dr. Petru Boișteanu, Professor dr. Vasile Chiriță, Conf. dr. Radu Andrei and Dr. M. E. Berlescu have revived the tradition of publications at "Socola" Hospital, editing the new "Bulletin of Integrative Psychiatry".

At the end of 2014, "Socola" Hospital became the "Socola" Institute of Psychiatry, which has increased its responsibilities regarding medical assistance, scientific research, didactic activity, professional training and also the development of editorial activity. 
Journal B+ CNCS and Indexed IDB by Index Copernicus, DOAJ, Erih Plus, Gale Cengage, CEEOL, Crossref

Editorial contact: contact@buletindepsihiatrie.ro

Publisher contact:

„Gr. T. Popa” Publisher

University of Medicine and Pharmacy Iaşi

$16^{\text {th }}$ Universităţii Str.

Tel. 0232301678

www.umfiasi.ro 


\section{Summary}

\section{Editorial / 11}

Reason and communication: digital form of communication in response to the COVID19 pandemic / 13

Laura Pricop

\section{Articles / 17}

A current perspective on the relevance of nano and microplastics in the neurodevelopmental disorders: further relevance for metabolic, gastrointestinal, oxidative stress-related and zebrafish studies / 19

Alexandrina Curpan, Ștefan Strungaru, Alexandra Săvucă, Ovidiu Ilie, Alin Ciobîcă, Daniel Timofte, Roxana Cojocariu, Gabriel Plăvan, Mircea Nicoară,Peiu Sorin Nicolae

The psychiatric impact of coronavirus infection in the population / 27

Leon-Constantin Maria Magdalena, Maștaleru Alexandra, Abdulan Irina Mihaela, Mitu Ovidiu, Oancea Andra, Zota Mădălina Ioana, Mitu Florin

The impact of COVID-19 infection of patients with autoimmune blistering diseases of the skin / 35

Adriana Popescu, Dumitrița-Lenuța Nichitean, Dan Vâță, Laura Stătescu, Ioana Alina Grăjdeanu, Elena Porumb-Andrese, Laura Gheucă Solovăstru

Is cardiovascular risk factor prevention necessary? The influence of diabetes mellitus in the development of Alzheimer's dementia / 39

Ioana A. Sandu, Anca I. Pîslaru, Adina C. Ilie, Ramona Ștefăniu, Călina A. Sandu, Anisia Alexa, Ioana D. Alexa, Cristinel Ștefănescu

Non-drug modalities of managing anxiety in dental office / 45

Georgiana Macovei, Alexandra Boloș, Magda Ecaterina Antohe, Gente Camille, Antonela Maria Beldiman

Clinical and biological particularities of depressive disorder in women patients / 51

Radu Gavril, Raluca Gavril, Irina Dobrin, Carmen Traian, Cristinel Ștefãnescu

Correlations between anxiety levels, gastrointestinal manifestations and social isolation following the lockdown due to coronavirus outbreak in Romanian students / 63

Alexandrina Curpan, Alexandra Săvucă, Luminița Hriscu, Alin Ciobîcă, Daniel Timofte,Peiu Sorin Nicolae 
Gabapentin: an old anticonvulsivant with new potential, may reduce withdrawal symptoms and contribute to alcohol abstinence / 71

Andreea Catalina Rusu, Ana Caterina Cristofor, George Catalin Morosan, Roxana Chiriță

A review on brain-derived neurotrophic factor and its role in depression and anxiety / 79

Vlad Teodor Iacob, Matei Palimariciuc, Vasile Chiriță, Roxana Chiriță

Hormonal effects of estrogen and progesterone in postpartum depression / 87

Roxana Mihaela Barbu, Cristina-Maria Gavrilescu, Elena Cojocaru, Răducu Ionuț Popescu, Daniela Ababei, Walther Bild

Case reports / 95

Particularities of a manic episode with psychotic symptoms on the background of psycho-trauma in adolescents $/ 97$

Bianca-Georgiana Secrieru, Elena-Rodica Popescu, Gabriela-Carmen Lupușoru, Roxana Chiriță

Book review / 105

The center cannot hold. The story of my madness / 107

The anti stigma manifesto of Elyn Saks

Instructions for authors / 111 


\section{Editorial}





\title{
Reason and communication: digital form of communication in response to the COVID-19 pandemic
}

\author{
Laura Pricop
}

\author{
Laura Pricop - PhD, researcher, Institute of Interdisciplinary Research, Alexandru Ion \\ Cuza University of Iași
}

The Covid 19 pandemic with its imposed measures of isolation and physical distancing had a major impact on people. In this editorial we recommend the use of the phrase physical distancing, much more appropriate than social distancing. Physical distancing involves a distance between two or more people in a social context. This distance cannot be less than 1.5 meters. This minimum distance can allow people to maintain both their privacy and the space needed to avoid physical contact, preserve their safety and at the same time give people the opportunity to be active in social contexts. Social distancing seems to have a dose of accentuated radicalism and implies isolation or self-isolation, i.e. a total distance from people and society.

These measures have transformed the lives of people everywhere, regardless of age or occupation. Physical distancing and isolation will continue to transform and affect our normal lives, even more as we are still looking for more effective treatment formulas and the development of a vaccine. Despite the efforts made, the number of new cases are increasing day by day worldwide, as well as the number of victims. There is still no clear solution to this complex of issues regarding the Sars-CoV-2 infection.

Humans are social beings and need to manifest themselves as such in the simplest ways that affirm their belonging to humanity: reason and communication. Even if most of the time the thought process is associated with the particular effort of silencing and distancing from the surrounding voices, in order to become reason, as Kant says, must be said and shared. Thinking becomes consistent only when it is brought into light and made known to others, discussed, challenged, questioned and understood, otherwise it is simply an empty exercise. The state of isolation and distancing between people has disrupted this normal movement of man throughout the world. 


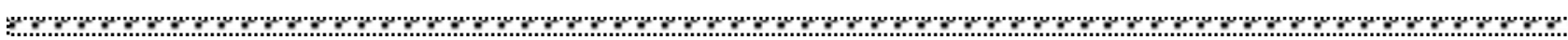

The individual's need for humanity (to be a student, to be a child, a doctor, a researcher or anything else, to live in these times of crisis, to live, to care and educate those in need, to make discoveries, and to find the most appropriate solutions) can only be supported by effective communication, which goes beyond the barriers imposed by isolation and physical distancing. A responsible, informative communication in a timely manner, is that able to eliminate ambiguities, leading to understanding, knowledge and educating.

Instinctively, people continued their activity working remotely, in the fields where this was and is possible, and the only thing that supported their remote activity was and continues to be communication. In this crisis situation, imposed by the COVID 19 pandemic, risk communication to the population and to all directly involved, doctor-patient communication and communication of the hospitalized and isolated patient and family, had to be reconfigured, taking into account the two barriers that are considered tools in stopping the spread of the virus: isolation and physical distance.

Online communication, asynchronous communication, social media or face-to-face communication through social platforms has replaced, with more or less success and where possible, in recent months traditional synchronous face-to-face communication. The synchronism, which the online environment favors, except for platforms that require the presence of all those involved in the discussion to be online in the same time frame, has led to the exacerbation of both the amount of information transmitted and that of false or questionable quality, which caused confusion and the misleading of users. This situation has reached alarming levels, prompting the director general of the World Health Organization, Tedros Adhanom Ghebreyesus, to state that: "We're not just fighting an epidemic; we're fighting an infodemic"(1)

As in a situation of crisis and uncertainty, the communicative reason has no other support than the facts, to our questions we must only find evidence-based answers provided by organizations, institutions that over time have proved their correctness in the reporting of facts.

Beyond these negative effects, the communication between doctor and nonhospitalized patient that requires a close follow-up or of the evolution and management of the disease or, between hospitalized and isolated patients and close ones and family, has been digitized to a large extent, in response to the measure of quarantining hospitals, closing medical offices, and banning the visit of hospitalized patients.

Telemedicine "has become essential to healthcare delivery in the COVID-19 era"(2) with video calls(3) being just an example of the digitization of communication. These new opportunities to communicate with patients come with serious qualitative limitations, but over time, these shortcomings can be at least mitigated.

Scientists, researchers, doctors, teachers understood the need to communicate more effectively, to share experiences with each other and despite the obstacles imposed by travel restrictions, organized virtual conferences, with virtual coffee breaks, virtual workshops, webinars. In order to make known to the opinion of the scientific 
Bulletin of Integrative Psychiatry O New Series OSeptember 2020 ○ Year XXVI ONo. 3 (86)/15

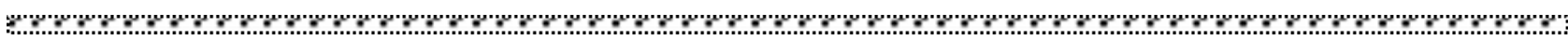
community and research results as soon as experiences in order to increase knowledge possible, many of the academic journals about this situation.

have changed their publishing policies. Some publishers have opted for a fast peer review system, for publishing articles in pre-print and/or open system format. They accepted short article formulas and some even created spaces for storing and presenting articles in the form of video recordings. These broad movements really indicate a variant of response to the crisis situation we are going through, affirming the need to accumulate facts, evidence, and

The policies of our magazine adopt an open line in support of an efficient and responsible communication with the stated purpose of supporting knowledge, to make known to those interested experiences, practices with results capable of bringing more knowledge to the current situation or to offer concrete, procedural solutions to particular situations.

\section{REFERENCES}

1. Zarocostas J. How to fight an infodemic. The Lancet.2020;395:10225.

2. 2.Ritchey K.C., Foy A., McArdel E., Gruenewald D.A. Reinventing palliative care delivery în the era of COVID-19: how telemedicine can supportend of life care. American Journal of Hospice Palliative Medicine.2020;37(11):992-997.

3. Negro A., Mucci M., Beccaria P., Borghi G., Capocasa T., Cardinali M., Pasculli N. et al. Introducing the Video call to facilitate the communication between health care providers and families of patients in the intensive care unit during COVID-19 pandemia. Intensive critical care nursing. 2020;60:102893. 



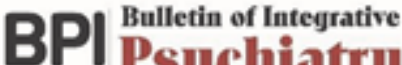

Psychiatry Euletin de Poíhiatrie Integrativa

\section{Articles}





\title{
A current perspective on the relevance of nano and microplastics in the neurodevelopmental disorders: further relevance for metabolic, gastrointestinal, oxidative stress-related and zebrafish studies
}

\author{
Alexandrina Curpan, Ștefan Strungaru, Alexandra Săvucă, Ovidiu Ilie, \\ Alin Ciobîcă, Daniel Timofte, Roxana Cojocariu, \\ Gabriel Plăvan, Mircea Nicoară, Peiu Sorin Nicolae
}

Alexandrina Curpan - Department of Research, Faculty of Biology, "Alexandru Ioan Cuza" University of Iasi, Carol I Avenue, 20A, Iași, Romania

Ştefan Strungaru - Department of Research, Faculty of Biology, "Alexandru Ioan Cuza" University of Iasi, Carol I Avenue, 20A, Iași, Romania

Alexandra Săvucă - Department of Research, Faculty of Biology, "Alexandru Ioan Cuza" University of Iasi, Carol I Avenue, 20A, Iași, Romania

Ovidiu Ilie - Department of Research, Faculty of Biology, "Alexandru Ioan Cuza" University of Iasi, Carol I Avenue, 20A, Iași, Romania

Alin Ciobîcă - Department of Research, Faculty of Biology, "Alexandru Ioan Cuza” University of Iasi, Carol I Avenue, 20A, Iași, Romania

Daniel Timofte - Faculty of Medicine, "Grigore T. Popa" University of Medicine and Pharmacy, 16th University Street, Iași, Romania

Roxana Cojocariu - Department of Research, Faculty of Biology, "Alexandru Ioan Cuza" University of Iasi, Carol I Avenue, 20A, Iași, Romania

Gabriel Plăvan - Department of Biology, Faculty of Biology, "Alexandru Ioan Cuza” University of Iași, Bd. Carol I, 20A, 700505 Iași, Romania

Mircea Nicoară - Department of Biology, Faculty of Biology, "Alexandru Ioan Cuza" University of Iași, Bd. Carol I, 20A, 700505 Iași, Romania

\section{ABSTRACT}

Modern world is relying more and more on the use of plastics in order to conduct our daily tasks going from the simple lid that covers our morning coffee to large scale use in packaging and buildings. But how does the use of so much plastic influence human's normal growth and development or more importantly, how does it affect the brain functions? For the purpose of this paper of giving a little literature insight over the toxic effects of micro-/nanoplastics we chose to approach it from a multidisciplinary point of view with a focus on the use of zebrafish as animal models; ranging from how microplastics disrupts the host eubiosis and 
\% gradually provokes an impairment along the gut-brain axis (GBA) in both mice and zebrafish to nanoplastics inducing oxidative stress status which furthermore leads to increased mortality and brain cells apoptosis. Nanoplastics has also been reported as being transferred from mother to offsprings causing a series of morphological and physiological changes, as well as neurotransmitters inhibitions (dopamine, oxytocin, serotonin) in zebrafish fish as several studies have suggested.

\section{KEYWORDS:}

Neuropsychiatric disorders, zebrafish, microplastics, gut, oxidative stress.

\section{INTRODUCTION}

Plastics consist of a large variety of polymer types, including polypropylene (PP), polyethylene (PE), polystyrene (PS), polyvinylchloride (PVC), polyethylene terephthalate (PET), polyamides (PA), and so on, which are mainly made from fossil fuels such as petroleum, natural gas, or coal, and are designed to meet the very different needs of end products. It is estimated that at least 8 million tons of plastic waste ends into the world's oceans each year and by 2050 the weight of marine plastics would exceed that of fish. The released plastics are generally subjected to progressive fragmentation under the comprehensive function of environmental physicochemical and biotic factors, such as mechanical abrasion, ultraviolet radiation, and biological degradation by microorganisms.

Micro-/nanoplastics are widespread contaminants. The human body is exposed to microplastics through ingestion of food containing microplastics, inhalation of microplastics in the air and by dermal contact of these particles, contained in products, textiles or in the dust. Ingestion is considered the major route of human exposure to micro/nanoplastics. Due to the evidence of neurotoxicity when testing microplastics in organism or cells and resulting from human exposure to particulate matter, which microplastics are a part of, there is a need to understand how microplastics could be involved in neurotoxicity in humans, contributing to an increased risk of neurodevelopmental disorders (1). These types of disorders represent a challenge for researchers at the worldwide level due to the difficulty of mimicking the whole symptomatology in a laboratory setting. Several neuropsychiatric disorders, such as depression, anxiety and autism $(2,3)$ were linked to the brain-gut impairments accompanied with functional gastrointestinal symptoms. Epilepsy has been induced in zebrafish larvae and adults by exposure to pentylenetetrazol (PTZ) (4), ginkgo toxin (PLP)(5) where it has been shown that there are changes in resting states that lead to hyperactivity and hypersynchronization of the brain (6) by changing GABA levels (5). Moreover, the pattern of co-occurrence of medical comorbidities in autism as compared to any other neurodevelopmental disorders is elevated, especially gastrointestinal disorders (7).

In recent years, a new animal model is gaining more ground in multiple medical and biological fields - zebrafish proving its utility and resemblance to the human nervous system by bringing to the table a series of more favorable features when compared to already established animal models, such as low upkeep cost, fast life cycle, long life (five years) as well as the amount of techniques available to genetically manipulate these vertebrates. Due to the high homology they present with humans (more than $70 \%$ of human genes have orthologues in zebrafish), the relevance of using it at different developmental stages has also improved, with embryos having the advantage of a translucent body and an ability to absorb substances by immersion while exhibiting movements as early as 17 hours post fertilization (muscular contractions) while at 48 hours post fertilization (hpf) they can fully 
respond to stimuli and are able to control their movements $(8,9)$.

Recent statements suggested that more than $99 \%$ of the human faeces samples originated from Asia and Europe contained microplastics. More studies should be conducted to explore the possibility of detecting microplastics in the human body and the effects of microplastics on human health. A reliable methodology for microplastics extraction with application in routine medical analyses is not developed yet. Such studies could raise awareness of the importance of sustainable development and lead to the sustainable development of food and water systems, environmental stewardship, and solutions to this global public health issue. However, there are no current studies focusing on the correlation between micro-/nanoplastics toxicity and neurodevelopmental disorders by the use of zebrafish animal models and human subject making this approach even more interesting.

\section{EFFECTS OF MICRO-/NANOPLASTICS IN HUMAN, MICE AND ZEBRAFISH}

A recent review on the human intake of MPs from food gave an estimate of 39,000-52,000 particles per year depending on age and gender (10). Tap water has been assumed to account for around $10 \%$ of this exposure (4000 particles per year). Consumption of exclusively (mineral) water from bottles would result in an additional exposure of approximately 90,000 MP particles per year. It has been indicated that the estimates should be seen as an underestimation. Combination of exposure from different sources would indicate a current human exposure exceeding 100,000 MP particles per capital during one year, depending on diet and geographic location, and on the particle size range included in the estimate. However, levels in food and water have been considered to be an underestimation. A set of 32 samples of bottled water from Bavaria (Germany) was investigated for the presence of MP particles of the size $1 \mu \mathrm{m}$ and larger (11). The counts of MP particles varied from 2649-2857 (mean \pm standard deviation) liter -1 in single use PET bottles and 4889-5432 liter -1 in water from reusable PET bottles to 6292-10521 liter -1 in glass bottles. Higher counts were found in older reused PET bottles than in new ones, but in all cases the diversity within each group was considerable. Particles smaller than $5 \mu \mathrm{m}$ were identified for approximately $96 \%$ in the PET bottles and $78 \%$ in the glass bottles. In order of abundance, PET (76\%) and PP (10\%) were most commonly found in water from the PET bottles. In glass bottles, PE (46\%), PP (23\%), and a copolymer of styrene and butadiene (14\%) were most common. Two studies have reported on the uptake and bioaccumulation of MPs in mice. In a study by Deng et al. 2017(12), fluorescently labelled PS MPs of 5 and $20 \mu \mathrm{m}$ were daily administered by oral gavage for 1 , $2,4,7,14,21$, and 28 days at a dose of 0.1 $\mathrm{mg}$ per day (corresponding to $1.46 \times 106$ and $2.27 \times 104$ particles 8 of 28 of the 5 and $20 \mu \mathrm{m}$ sizes, respectively). Wash-out groups were exposed for 28 days and sacrificed after a week without treatment. Using light and fluorescence microscopy, MPs were detected in various tissues. Liver, kidney, and gut tissue material was also digested using nitric acid and hydrogen peroxide, and fluorescence in these samples was measured to quantify MP concentrations. These measurements demonstrated gradual accumulation in all tissues that reached a steady state at around 14 days of exposure. After 28 days of exposure, concentrations in the liver, kidney, and gut for the $5 \mu \mathrm{m}$ MPs were reported to be $0.077,0.099$, and $0.417 \mathrm{mg}$ g-1 (ww), respectively, and for the $20 \mu \mathrm{m}$ MPs 0.194, 0.082 , and $0.234 \mathrm{mg} \mathrm{g}-1$ (ww), respectively (levels based on wet weight were provided by Deng and Zhang(12)). After a wash-out period of 1 week, MPs could still be detected in all tissues (levels not reported). An important issue is whether MPs can be absorbed and enter the systemic circulation. Various reports mention that particles $150 \mu \mathrm{m}$ in size or smaller have the potential to cross the intestinal barrier of mammals, whereas particles $20 \mu \mathrm{m}$ in size or smaller may have the potential to penetrate deeply into tissues. Phagocytosis and endocytosis have been named as mechanisms for the uptake of particles smaller than $10 \mu \mathrm{m}$ and persorption in the Peyer's patches of the ileum for 
\%

particles up to $130 \mu \mathrm{m}$ in size has been found, although a low uptake of $0.002 \%$ per $24 \mathrm{~h}$ was reported for the latter process $(13,14)$. When MPs enter tissues, potential effects might include physical stress and damage, apoptosis, necrosis, inflammation, oxidative stress, and immune responses. Diffusion of monomers, toxic substances, or microbes from the particles to the surrounding tissue might contribute to chemical and microbiological hazards. Exposure of zebrafish to MPs [PS; $5 \mu \mathrm{m}$; control, low (50 $\mu \mathrm{g}$ liter-1) and high (500 $\mu \mathrm{g}$ liter-1) exposure] has been found to result in distribution in the gut tissue, as evaluated by histological analysis. Furthermore, inflammatory responses, oxidative stress, and changes in lipid metabolism in the gut tissue have been reported (15).

The antagonistic effect of MPs on the gut microbiota promotes significant long-term repercussions. Not only disrupts the host eubiosis, but also gradually provokes an impairment along the gut-brain axis (GBA) in both mice and zebrafish. Through the analysis of the bacterial hypervariable regions V3-V4 of the 16 rRNA gene, it has been revealed a disruption of the so-called beneficial bacteria in four distinct phyla such as Bacteroidetes, Proteobateria, Firmicutes and Actinobacteria. Subsequent in-depth analysis revealed that twenty-nine intestinal microorganisms identified by operational taxonomic analysis (OUT) changed, of which fifteen at the genus level during exposure to MP. MPs have induced an increased mRNA level of several pro-inflammatory cytokines which explains the metabolic disorders as a result of a dysfunction of the intestinal barrier $(16,17)$.

Continuing this topic and analogous to previous studies, intestinal dysbiosis is associated with fluctuations in mucus volume. A study conducted by Lu et al., 2018(18) has provided a more deepen perspective at the phylum level, MPs being positively correlated with a low richness of Firmicutes and $\alpha$ Proteobacteria following the analysis of the $\mathrm{V} 3-\mathrm{V} 4$ region of the 16 rRNA gene and the overall diversity. Between 310 and 160 gut microbes have been changed after the exposure to MPs, by leading to a decrease of the hepatic triglyceride (TG) and total cholesterol (TCH) level in mice, reflected by a high predisposition towards hepatic lipid disorders.

According to current literature regarding the use of zebrafish embryo and larvae, most studies have been conducted for toxicological assays, but the material and methods are quite similar therefore can be used for studies with a different desired outcome.

With this being said, zebrafish has been used in order to assess the toxic effects of nanoplastics (NPs) on the living organisms, plankton and aquatic life in general. Even if most studies focused on the environmental effects of MPs, over the last years NPs had become a researched topic as "smaller particles are generally more toxic than the corresponding bulk material at the same mass concentration"(19). Studies using $20 \mathrm{~nm}$ diameter polystyrene nanoplastics (PNP) have managed to illustrate the oxidative stress induced by the accumulation of the particles in the brain leading to increased mortality and brain cells apoptosis, illustrating as well their ability to cross the blood-brain barrier - with oxidative stress being one of the possible underlying theories of neurodevelopmental disorders. When applied to other animal models, it was suggested that PNP can affect the nervous system with focus on the behavioral changes, like reduced motor activity and severe seizure-like behavior (20, 21). Several studies have demonstrated that NPs can be transmitted from mother to offspring causing morphological and physiological alterations such as increased mortality in embryo and increased yolk area, spine curvature, caudal flexure etc. in larvae as well as decreased cyclooxygenase (COX) activity in embryo and inhibition of a series of neurotransmitters (dopamine - thought to be also involved in schizophrenia, oxytocin, serotonin, melatonin, low acetylcholinesterase activity). In larvae, NPs can accumulate in mouth, gut, blood, liver, heart, gills, muscles and brain, but the interaction of NPs in the intestinal tract affects the gut microbiome and leads to inflammation (gastrointestinal system of zebrafish is homologous to the mammalian 


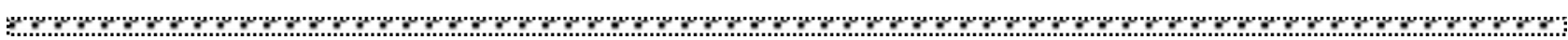

one), concomitantly with a disruption of the gut metabolism through an abnormal gene expression and energy production (22).

Studies using zebrafish have opened up new perspectives as it turned out that recurrent light-sensitive effects (23) and some parameters increased with exposures to PTZ concentrations that led to the cumulative frequency of the score, the intensity of seizures and their severity, latency to onset, mortality rate $(6,24)$ all opening avenues of research for new genetic markers of epilepsy in zebrafish (25).

As previously stated, zebrafish are capable of exhibiting complex behavior as early as 48 hours post fertilization corresponding to the larvae stage. Similar to other animal models, the behavior of this teleost can be assessed with a plethora of tests such as the light-dark test, novel tank test for anxiety with the unique behavior of freezing and diving to the bottom, T-maze, plus maze and social preference, as well as the acoustic startle reflex that is used to test the ability to learn as well as memory based on the habituation ability represented in this case by a rapid escape response - C-start, which is sensible to $\mathrm{N}$-methyl-D-aspartate receptor (NMDAR) blockade (neurotransmitter involved in schizophrenia's etiology). Another test that can be conducted on zebrafish larvae that is also used to test the short- and long-term memory of zebrafish is visual lateralization (novel object recognition test) with zebrafish having a preference of using left eye system for appraising novelty. The last two tests being specific for this animal model.

Whether it is about the way of communication through the vagus nerve (26), the immune(27) or neuroendocrine pathways (28), or even microorganism-derived neuroactive chemicals (29), the undoubted connection between gut and brain has been the subject of countless studies.

It has been shown that the bidirectional braingut axis disturbance could be involved in the abnormal function of the enteric or/and central nervous systems (CNS), this being an additional argument that links CNS to mechanisms that may have a role in the pathogenesis of functional gastrointestinal disorders (30). Regarding one of them, irritable bowel syndrome (IBS), it has been shown that stress plays an essential role in the occurrence of its symptoms, given the fact that it can modulate the hypothalamicpituitary-adrenal (HPA) axis activity $(31,32)$. Also, inflammatory bowel disease (IBD), which is a chronic relapsing disorder with a progressive and destructive nature (33), is linked to risk factors such as microbial dysbiosis, or exposure to toxins including xenoestrogens. In this regard, recent findings indicated that bisphenol-A (BPA), which is usually found in polycarbonate plastics and epoxy resins, is correlated with colonic inflammation. Moreover, BPA not only has the ability to exacerbate colonic inflammation, but alter microbiota metabolites derived from aromatic amino acids in an acute dextran sulfate sodiuminduced colitis animal model, increasing mortality and aggravating disease activity as well as inflammation in the middle colon (34).

Even if the exact communication pathways mechanism between the brain and gut are still unclear, it seems that the gut microbiome influences brain and behavior, using microbiota-gut-brain axis $(35,36)$, in this way, understanding why the microbiome influences behavior requires a focus on microbial ecology and local effects within the host. The exact underlying mechanism of how the homeostasis of this microscopic environment is gradually disrupted in humans is obscure. Unfortunately, in the current literature no study that addresses such a perspective exists, everything remaining at the stage of hypothesis. However, one pilot study has provided conclusive evidence in this context, and indeed demonstrated the existence of MPs in the human stool, which could constitute the missing link (37).

In fact, our group is currently working on further understanding and establishing the potential of zebrafish embryos and larvae in the study of neurodevelopmental disorders, as 
r.m\%\%

well as studying the current interactions between nanoplastics, microbiota changes and neurodevelopmental disorders and their importance, understanding the connection between micro-/nanoplastics pollution and physiological and behavioral changes and also establishing oxidative stress status disruption that was observed in schizophrenia, autism and MPs/NPs toxicity assays in order to assess its implications in the etiology of these disorders.

\section{CONCLUSION}

The need to reduce our plastic usage becomes more critical as days are passing by and more studies are bringing to light the harmful effects of plastics at a micro and nano level. Studies using zebrafish have opened up new perspectives due to their anatomical similarities to human and rodents, especially the existence of the blood-brain barrier.

Even if a direct, clean-cut connection can't be established between micro-/nanoplastics pollution and neuropsychiatric disorders, a more indirect one could be observed by the effects of plastics on gut microbiota as brain-gut impairments has been previously linked to several neuropsychiatric disorders such as depression, anxiety and autism, even by our team. The hypothesis that oxidative stress might be an underlying mechanism of psychiatric disorders such as schizophrenia and autism and the observation that plastics can induce a disruptance of the oxidative status requires further investigations and studies on whether plastics induce neuropsychiatric disorders by impairments at the oxidative status and gut level or not, and we recommend the use of zebrafish for this purpose.

\section{ACKNOWLEDGEMENTS AND DISCLOSURES}

Ciobîcă A. is currently supported by a UEFISCDI Research Grant for Young Research Teams Support, number PN-III-P1-1.1-TE2016-1210, named "Complex study on oxidative stress status, inflammatory processes and neurological manifestations correlations in irritable bowel syndrome pathophysiology (animal models and human patients)." Also, Cojocariu RO was funded by the European Social Fund, through Operational Programme Human Capital 2014-2020, project number POCU/380/6/13/123623, project title "PhD Students and Postdoctoral Researchers Prepared for the Labour Market!"

\section{REFERENCES}

1. Prata JC, da Costa JP, Lopes I et al. Environmental exposure to microplastics: An overview on possible human health effects. Sci Total Environ. 2020.1;702. Available from: https://pubmed.ncbi.nlm.nih.gov/31733547/

2. O'malley D, Quigley EMM, Dinan TG, Cryan JF. Do interactions between stress and immune responses lead to symptom exacerbations in irritable bowel syndrome? Brain Behav Immun. 2011;25(7):1333-41. https://pubmed.ncbi.nlm.nih.gov/21536124/

3. Singh P, Agnihotri A, Pathak MK et al. Psychiatric, somatic and other functional gastrointestinal disorders in patients with irritable bowel syndrome at a tertiary care center. J Neurogastroenterol Motil. 2012;18(3):324-31. https://pubmed.ncbi.nlm.nih.gov/22837881/

4. Burrows DRW, Samarut, Liu J et al. Imaging epilepsy in larval zebrafish. Eur J Paediatr Neurol. 2020;24:70-80. https://doi.org/10.1016/j.ejpn.2020.01.006

5. Lee GH, Sung SY, Chang WN et al. Zebrafish larvae exposed to ginkgotoxin exhibit seizure like behavior that is relieved by pyridoxal-5'-phosphate, GABA and anti-epileptic drugs. DMM Dis Model Mech. 2012;5(6):785-95.

6. Diaz Verdugo C, Myren-Svelstad S, Aydin E et al. Glia-neuron interactions underlie state transitions to generalized seizures. Nat Commun. 2019;10(1):1-13.

7. Pulikkan J, Mazumder A, Grace T. Role of the Gut Microbiome in Autism Spectrum Disorders. Adv Exp Med Biol. 2019;1118:253-69. 
2. 8. Spence R, Gerlach G, Lawrence C, Smith C. The behaviour and ecology of the zebrafish, Danio rerio. Biol Rev. 2008;83(1):13-34.

9. Vaz R, Hofmeister W, Lindstrand A. Zebrafish Models of Neurodevelopmental Disorders: Limitations and Benefits of Current Tools and Techniques. Int J Mol Sci. 2019;20(6):1-26.

10. Cox KD, Covernton GA, Davies HL et al. Human Consumption of Microplastics. Environ Sci Technol. 2019;53(12):7068-74.

11. Oßmann BE, Sarau G, Holtmannspötter H et al. Small-sized microplastics and pigmented particles in bottled mineral water. Water Res. 2018;141:307-16.

12. Deng Y, Zhang Y, Lemos B, Ren H. Tissue accumulation of microplastics in mice and biomarker responses suggest widespread health risks of exposure. Sci Rep. 2017;7(1):1-10.

13. Revel M, Châtel A, Mouneyrac C. Micro(nano)plastics: A threat to human health? Curr Opin Environ Sci Heal. 2018;1:17-23.

14. Academies SA for P by E. A Scientific Perspective on Microplastics in Nature and Society. Berlin; 2019. https://www.sapea.info/wp-content/uploads/report.pdf

15. Qiao R, Sheng C, Lu Y et al. Microplastics induce intestinal inflammation, oxidative stress, and disorders of metabolome and microbiome in zebrafish. Sci Total Environ. 2019;662:246-53.

16. Jin Y, Xia J, Pan Z et al. Polystyrene microplastics induce microbiota dysbiosis and inflammation in the gut of adult zebrafish. Environ Pollut. 2018;235:322-9.

17. Jin Y, Lu L, Tu W et al. Impacts of polystyrene microplastic on the gut barrier, microbiota and metabolism of mice. Sci Total Environ. 2019;649:308-17.

18. Lu L, Wan Z, Luo T et al. Polystyrene microplastics induce gut microbiota dysbiosis and hepatic lipid metabolism disorder in mice. Sci Total Environ. 2018;631-632:449-58.

19. Mattsson K, Johnson E V., Malmendal A et al. Brain damage and behavioural disorders in fish induced by plastic nanoparticles delivered through the food chain. Sci Rep. 2017;7(1):1-7.

20. Sökmen TÖ, Sulukan E, Türkoğlu M et al. Polystyrene nanoplastics $(20 \mathrm{~nm})$ are able to bioaccumulate and cause oxidative DNA damages in the brain tissue of zebrafish embryo (Danio rerio). Neurotoxicology. 2020;77:51-9.

21. Bhagat J, Zang L, Nishimura N, Shimada Y. Zebrafish: An emerging model to study microplastic and nanoplastic toxicity. Vol. 728, Science of the Total Environment. Elsevier B.V.; 2020. p. 138707.

22. Wan Z, Wang C, Zhou J et al. Effects of polystyrene microplastics on the composition of the microbiome and metabolism in larval zebrafish. Chemosphere. 2019;217:646-58.

23. Sun S, Zhu C, Ma M et al. A pentylenetetrazole-induced kindling zebrafish larval model for evoked recurrent seizures. bioRxiv. 2019;787580.

24. Mussulini BHM, Leite CE, Zenki $\mathrm{KC}$ et al. Seizures Induced by Pentylenetetrazole in the Adult Zebrafish: A Detailed Behavioral Characterization. Callaerts P, editor. PLoS One. 2013;8(1):e54515.

25. Stewart AM, Desmond D, Kyzar E et al. Perspectives of zebrafish models of epilepsy: What, how and where next? Brain Res Bull. 2012;87(2-3):135-43.

26. Forsythe P, Bienenstock J, Kunze WA. Vagal Pathways for Microbiome-Brain-Gut Axis Communication BT Microbial Endocrinology: The Microbiota-Gut-Brain Axis in Health and Disease. In: Lyte M, Cryan JF, editors. New York, NY: Springer New York; 2014.115-33.

27. Fung TC, Olson CA, Hsiao EY. Interactions between the microbiota, immune and nervous systems in health and disease. Nat Neurosci. 2017;20(2):145-55.

28. Neuman H, Debelius JW, Knight R, Koren O. Microbial endocrinology: the interplay between the microbiota and the endocrine system. FEMS Microbiol Rev. 2015;39(4):509-21.

29. Lyte M. Microbial endocrinology in the microbiome-gut-brain axis: how bacterial production and utilization of neurochemicals influence behavior. PLoS Pathog. 2013/11/14. 2013;9(11):e1003726-e1003726.

30. Karantanos T, Markoutsaki T, Gazouli M et al. Current insights in to the pathophysiology of Irritable Bowel Syndrome. Gut Pathog. 2010;2(1):3.

31. Musial F, Häuser W, Langhorst J. Psychophysiology of visceral pain in IBS and health. J Psychosom Res. 2008;64(6):589-97.

32. Qin HY, Cheng CW, Tang XD, Bian ZX. Impact of psychological stress on irritable bowel syndrome. World J Gastroenterol. 2014;20(39):14126-31.

33. Neurath MF. Current and emerging therapeutic targets for IBD. Nat Rev Gastroenterol Hepatol. 2017;14(5):26978. 

34. DeLuca JAA, Allred KF, Menon R et al. Bisphenol-A alters microbiota metabolites derived from aromatic amino acids and worsens disease activity during colitis. Exp Biol Med. 2018;243(10):864-75.

35. Crumeyrolle-Arias M, Jaglin M, Bruneau A et al. Absence of the gut microbiota enhances anxiety-like behavior and neuroendocrine response to acute stress in rats. Psychoneuroendocrinology.2014;42:207-17.

36. Foster JA, Rinaman L, Cryan JF. Stress \& the gut-brain axis: Regulation by the microbiome. Neurobiol Stress. 2017;7:124-36.

37. Liebmann B, Köppel S, Königshofer P et al. Assessment of microplastic concentrations in human stool Preliminary results of a prospective study. 2018.

\section{Correspondence}

Daniel Timofte,

$\mathrm{MD}, \mathrm{PhD}$, professor, Faculty of Medicine, "Grigore T. Popa” University of Medicine and Pharmacy, 16th University Street, Iasi, Romania, dantimofte@ yahoo.com

Submission: 20 jul. 2020

Acceptance: 02 sep. 2020 


\title{
The psychiatric impact of coronavirus infection in the population
}

\author{
Leon-Constantin Maria Magdalena, Maștaleru Alexandra, \\ Abdulan Irina Mihaela, Mitu Ovidiu, Oancea Andra, \\ Zota Mădălina Ioana, Mitu Florin
}

\begin{abstract}
Maria-Magdalena Leon-Constantin - "Grigore T Popa” University of Medicine and Pharmacy Iasi, Romania, Faculty of Medicine, Department of Medical Specialties (I), address: University street nr 16, Iasi, Romania; Clinical Rehabilitation Hospital - Cardiovascular Rehabilitation Clinic Alexandra Maștaleru - "Grigore T Popa" University of Medicine and Pharmacy - Iasi, Romania, Faculty of Medicine, Department of Medical Specialties (I), address: University street nr 16, Iasi, Romania; Clinical Rehabilitation Hospital - Cardiovascular Rehabilitation

Irina Mihaela Abdulan - "Grigore T Popa" University of Medicine and Pharmacy - Iasi, Romania, Faculty of Medicine, Department of Medical Specialties (I), address: University street nr 16, Iasi, Romania; Clinical Rehabilitation Hospital - Cardiovascular Rehabilitation Clinic

Ovidiu Mitu - "Grigore T Popa" University of Medicine and Pharmacy - Iasi, Romania, Faculty of Medicine, Department of Medical Specialties (I), address: University street nr 16, Iasi, Romania; Sf. Spiridon Hospital - Cardiology Clinic

Andra Oancea - "Grigore T Popa" University of Medicine and Pharmacy - Iasi, Romania, Faculty of Medicine, Department of Medical Specialties (I), address: University street nr 16, Iasi, Romania; Clinical Rehabilitation Hospital - Cardiovascular Rehabilitation Clinic

Madalina Ioana Zota - "Grigore T Popa" University of Medicine and Pharmacy - Iasi, Romania, Faculty of Medicine, Department of Medical Specialties (I), address: University street nr 16, Iasi, Romania; Clinical Rehabilitation Hospital - Cardiovascular Rehabilitation

Florin Mitu - "Grigore T Popa" University of Medicine and Pharmacy - Iasi, Romania, Faculty of Medicine, Department of Medical Specialties (I), address: University street nr 16, Iasi, Romania; Clinical Rehabilitation Hospital - Chief of Cardiovascular Rehabilitation Clinic
\end{abstract}

\section{ABSTRACT}

The Coronavirus pandemic that started last year has an unsuspected magnitude, spreading worldwide. In addition to physical suffering, patients confirmed or suspected of COVID-19 face severe psychological pressures. Isolated, suspicious cases may suffer from anxiety due to uncertainty about their health and may develop obsessive-compulsive symptoms, such as repeated temperature checks, sterilization or sanitization of surrounding objects. People with 
\% psychiatric disorders are more likely to develop severe episodes of depression, anxiety, insomnia stress and even forms of post-traumatic stress. In the face of these new critical situations, front-line medical staff, who are directly involved in the diagnosis, treatment and care of patients with COVID-19, risk developing forms of psychiatric suffering. Unfortunately, medical staff also face numerous phobias imposed by restrictive measures in the context of the pandemic: hand disinfection, wearing personal protective equipment, disposable gowns, etc. Also, another phobia of the medical staff is the distancing from the family or the risk of illness. Emerging mental health issues related to this global event can evolve into long-term health problems, isolation and stigma. Telepsychiatry and behavioral therapy through smart devices should focus on relaxation exercises in order to counteract anxiety, post-traumatic depression-like symptoms, anger and irritability. Planning activities (for example, exercising at home) can improve your physical and mental health. COVID-19 infection is a global problem that has caused a radical change in lifestyle, the impact of these changes, restrictions and major changes being received differently depending on the character of each person. This pandemic seems far from over. We hope that the number of patients with mental illness will not increase exponentially and through alternative methods we will maintain the well-being of those who exist.

\section{KEYWORDS:}

Coronavirus, anxiety, depression.

\section{INTRODUCTION}

The Coronavirus Pandemic (SARS-CoV-2), which initially occurred in Wuhan, China, has spread nationally and then worldwide. The application of strict quarantine measures, unprecedented so far, has kept a large number of people isolated and affected many aspects of people's lives around the world.

In the last months, following the outbreak of the pandemic, an increasing number of information have had an impact on global mental health. The media, local and international health organizations (including the World Health Organization), epidemiologists, virologists, and opinion leaders present data, recommendations and updates on the spread and lethality of SARSCoV-2 (1). The burden of this infection on global mental health is often neglected, risking to contaminate more and more people in the general population.

\section{INFECTED PATIENTS}

In addition to physical pain, patients confirmed or suspected of COVID-19 face high psychological pressures as well as other health problems. Confirmed and suspected cases of COVID-19 may have fears about the consequences of the disease as well as its contagion. Consequently, they may experience episodes of loneliness, denial, anxiety, depression, insomnia and despair, which may influence therapeutic compliance (2).

Isolated suspected cases may suffer from anxiety due to uncertainty about their health and may develop obsessive-compulsive symptoms, such as repeated temperature checks and sterilization or sanitization of surrounding objects. In addition, a strict policy of quarantine and follow-up by health authorities could lead to rejection by society, considerable financial losses, discrimination and stigma $(3,4)$. Also, limited knowledge of 
this virus and overwhelming news can lead to anxiety and agoraphobia (5).

COVID-19 appears to affect central nervous system function, which may indicate that neuropsychiatric symptoms may occur in the general population. A retrospective study that included patients with COVID-19 $(\mathrm{n}=214)$ found that manifestations of the central nervous system (dizziness, headache or impaired consciousness) occurred in $25 \%$ of cases (6).

Another particularly important aspect is that psychiatrists perform consultations in patients receiving pharmacotherapy for COVID-19. However, the side effects of psychiatric medication for COVID should not be neglected. As an example, hydroxychloroquine, one of the most widely used drugs for hospitalized COVID-19 patients, is associated with psychiatric side effects, such as mood and anxiety disorders, insomnia, and, more rarely, psychosis (7).

\section{PSYCHIATRIC PATIENTS}

People with psychiatric disorders are more likely to develop severe episodes of depression, anxiety, insomnia, stress and even forms of post-traumatic stress. At the same time, the difficulty in accessing health services during the pandemic favored the underlying disease. However, the immediate mental health care needs of these patients were considered not to be an emergency when the number of COVID-19 cases increased sharply. Then, patients were encouraged not to go to the hospital, as health services were dedicated to the management of terminally ill patients and cases suspected or confirmed by COVID-19. Quarantine / isolation measures have also hampered patients' access to psychiatric practices as well as other health care providers either due to insufficient medical resources or fear of contracting
COVID-19 in hospitals that have managed infected patients (8).

However, certain measures are needed to support this category of patients, especially those with suicidal ideation. Improved access to tele-psychiatric services, home delivery of psychotropic drugs and online psychiatric first aid resources play a key role in minimizing the severity of psychiatric symptoms $(8,9)$.

\section{CLINICIANS}

Healthcare professionals are no exception when it comes to the impressive impact of this pandemic on mental health, as many of them have direct contact with infected patients, close contact with patients' families / relatives, and are sometimes involved in epidemiological investigations.

A notable example would be the psychological trauma observed during the SARS epidemic in 2003 when $27 \%$ of the medical staff reported psychiatric symptoms (10). Similar effects appeared in 2015 during the Korean MERS epidemic, when medical staff showed symptoms of PTSD (11).

In the face of these new critical situations, front-line healthcare professionals who are directly involved in the diagnosis, treatment and care of patients with COVID-19 are at risk of developing forms of psychiatric suffering. The growing number of confirmed and suspicious cases, the overwhelming workload, the exhaustion of protective equipment, the lack of specific treatment and the intense media coverage of the problem can all contribute to their mental burden (12).

Recent studies show that in emergency units, Wuhan medical staff faced a high risk of infection and inadequate protection against contamination, overload, feelings of 
к

frustration, discrimination, isolation and exhaustion (13).

These problems not only affect the attention, understanding and decision-making capacity of medical staff, which could affect the fight against COVID-19, but could also have a lasting effect on their general condition (14).

Another cross-sectional study that included 1257 subjects in China revealed a high prevalence of psychiatric symptoms among medical staff treating patients with COVID19. Depression (50.4\%), anxiety (44.6\%), insomnia (34\%) and stress (71.5\%) were the main manifestations discovered. Most of the participants were women, nurses, aged between 26 and 40, married. They are likely to be at the highest risk of infection due to their close and frequent contact with patients as well as numerous shifts (15).

Another cross-sectional study evaluated nearly 300 physicians and nurses who cared for patients with COVID-19 in Singapore from February 19 to March 13, 2020, using self-reporting screening tools. Relatively few physicians reported anxiety, depression, and post-traumatic stress disorder (PTSD) (approximately 5-10), results that the authors attributed to better training of clinicians, based on their previous experience with the 2003 SARS epidemic (16).

\section{GENERAL POPULATION}

The almost exclusive focus on transmitting COVID-19 infection worldwide may distract the public from the psychosocial consequences for both affected individuals and the general population. Emerging mental health issues related to this global event can evolve into long-term health problems, isolation and stigma. Global measures should include measures to reduce psychosocial stressors, in particular in relation to isolation / quarantine, fear and vulnerability among the general population.

The mentioned manifestations among the medical staff were also found in the general population. Studies show that the prevalence of PTSD was between $4 \%$ and $41 \%$; the prevalence of major depression increased by $7 \%$ after the outbreak. There are several factors that can increase the risk of developing the conditions described: gender, poor socio-economic status, the existence of interpersonal conflicts, frequent use of social media and low social support (17).

During each community crisis, people often look for information about ongoing events in an attempt to keep up to date. However, when information on official channels is missing or irregularly broadcast, people can be exposed to misleading data from social media and the media. This underscores the importance of formal updates at regular intervals and the need to monitor information to reduce exposure to misleading news and stress. In fact, the fear of the unknown leads to a higher level of anxiety in both healthy people and those with pre-existing mental health problems; unjustified public fear can lead to discrimination and stigmatization (18).

First, people's emotional responses are likely to include extreme fear and uncertainty, and negative social behaviors will often be driven by fear and distorted perceptions of risk. Second, special efforts should be directed at vulnerable populations, including infected and sick patients, their families and colleagues, individuals and their relationships with the community, people with pre-existing medical conditions (both physical and / or mental), providers of medical services, in particular nurses and doctors working directly with sick or quarantined people (19). 
Patients with chronic diseases (cardiovascular disease, chronic kidney disease, diabetes mellitus) need regular medical monitoring. In this time of crisis, patients may also have difficulty getting maintenance treatment. As a result, preventive measures can lead to decreased physical well-being, which increases the risk of negative feelings and suicide (20).

The families of the deceased patients also suffer from the feeling of helplessness, the impossibility to say goodbye to the deceased relatives. The religious factor is all the more important as the funeral services have also been adapted to the current conditions of social distancing, preventing the organization of the services according to traditions (21).

\section{PATIENTS MANAGEMENT}

If possible, psychiatric consultations should be conducted by video conference or telephone, rather than face-to-face. The impact on the patient with the doctor's voice or image may be greater than text messages and emails. When face-to-face visits are required, personal protective equipment (eg masks) should be used and ideally, the consultation should be performed with a safe patient, avoiding group therapy $(20,22)$.

Given traffic limits and quarantine measures in many areas, online mental health services have been widely adopted, such as hotlines and mobile application platforms. Psychological care services, including counseling or intervention by telephone, internet and applications, need to be widely adopted by local and national mental health institutions in response to the COVID-19 outbreak. (23).

Telepsychiatry and behavioral therapy through smart devices should focus on relaxation exercises in order to counteract anxiety, PTSD-like symptoms, anger and irritability (24). Planning activities (for example, exercise at home) can improve your physical health (25).

Due to the prolonged blockade and closure of businesses, people face social isolation, lifestyle disruption and loss of personal income, while society loses its productivity in an inactive economy. Returning to work and reducing the spread of COVID-19 will improve self-esteem, financial situation and rebuild the social connection, while improving society's productivity, leading to a better quality of life and a lower level of depression and stress (26).

A recent article that included 24 papers that studied the psychological impact of quarantine on the population suggested the following steps to mitigate the negative effects: clarifying the purpose of quarantine and measures to be followed; understanding that a voluntary quarantine is associated with fewer side effects compared to a mandatory quarantine; emphasizing the altruistic benefit of quarantine by keeping others (especially those close to but not only) safe; finding the easiest way to purchase general purpose items (eg food and household necessities) as well as medical items (eg, prescriptions, thermometers, and masks) (27).

The difficulties posed by the COVID-19 pandemic, including isolation at home, economic hardship and limited accessibility to medical and mental health care, can give rise to suicidal ideation and behavior. Previous epidemics and pandemics have also reported suicide as an side effect of quarantine (28).

People diagnosed with moderate to severe anxiety or depression should be reevaluated during this period for suicidal thoughts and behavior; there are various ways in which this 
r.\%\% screening can be done (from specialized tools laptop / telephone, as well as face-to-face to a simple question). Patients with COVIDmeetings that maintain physical distance. In 19 and patients with established mental disorders should be encouraged to maintain social contact by telephone or computer / addition, patients should be encouraged to access telemedicine treatment (29).

\section{CONCLUSIONS}

COVID-19 infection is a global problem that has led to a radical change in lifestyle. Unfortunately, this pandemic is not over and we do not know how long it will last. Their impact changes, restrictions, major changes are received differently depending on the character of each person. It should be noted that people with psychiatric illnesses need better supervision and counseling during the crisis. As we expected, the number of these patients has increased and new patients need to be helped and protected from external stress. Discussions through tele-medicine are an excellent idea as they manage to treat the cases but also to calm the patient.

\section{ACKNOWLEDGEMENTS AND DISCLOSURES}

The authors declare that they have no potential conflict of interest to disclose.

\section{REFERENCES}

1. Organization WH. 2020. WHO characterizes COVID-19 as a pandemic. World Heatlh Organization https://www.who.int/emergencies/diseases/novel-coronavirus-2019/events-as-they-happen. Published 2020.

2. Xiang Y-T, Yang Y, Li W, Zhang L, Zhang Q, Cheung T. Timely mental health care for the 2019 novel coronavirus outbreak is urgently needed. Lancet Psychiatry. 2020: In press.

3. Shigemura J, Ursano RJ, Morganstein JC, Kurosawa M, Benedek DM. Public responses to the novel 2019 coronavirus (2019-nCoV) in Japan: mental health consequences and target populations. Psychiatry and Clinical Neurosciences. 2020: In press

4. Kar SK, Yasir Arafat SM, Kabir R, Sharma P, Saxena SK. Coping with Mental Health Challenges During COVID-19. Coronavirus Disease 2019 (COVID-19). 2020;199-213.

5. Bao Y, Sun Y, Meng S, Shi J, Lu L. 2019-nCoV epidemic: address mental health care to empower society. Lancet (London, England). 2020: In press

6. Troyer EA, Kohn JN, Hong S. Are we facing a crashing wave of neuropsychiatric sequelae of COVID-19? Neuropsychiatric symptoms and potential immunologic mechanisms. Brain Behav Immun 2020. In press.

7. Mascolo A, Berrino PM, Gareri $P$ et al. Neuropsychiatric clinical manifestations in elderly patients treated with hydroxychloroquine: a review article. Inflammopharmacology. 2018;26(5):1141-1149.

8. Fengyi Hao, Wanqiu Tan, Li Jiang et al. Do psychiatric patients experience more psychiatric symptoms during COVID-19 pandemic and lockdown? A case-control study with service and research implications for immunopsychiatry. Brain Behav Immun. 2020

9. Khanna R, Forbes M. Telepsychiatry as a public health imperative: Slowing COVID-19. Aust N Z J Psychiatry. 2020 [Epub ahead of print]

10. McAlonan GM, Lee AM, Cheung V. Immediate and sustained psychological impact of an emerging infectious disease outbreak on health care workers. Can J. Psychiat. 2007;52(4):241-247.

11. Lee SM, Kang WS, Cho AR, Kim T, Park JK. Psychological impact of the 2015 MERS outbreak on hospital workers and quarantined hemodialysis patients. Comprehensive Psychiatry. 2018; 87:123-127.

12. Moesmann Madsen M, Dines D, Hieronymus F. Optimizing psychiatric care during the COVID-19 pandemic. Acta Psychiatr Scand. 2020 [Epub ahead of print]

13. Kang L, Li Y, Hu S, Chen M, Yang C, Yang B. The mental health of medical workers in Wuhan, China dealing with the 2019 novel coronavirus. The Lancet Psychiatry. 2020; 7:E14.

14. Jones NM, Thompson RR, Dunkel Schetter C, Silver RC. Distress and rumor exposure on social media during a campus lockdown. Proceedings of the National Academy of Sciences of the United States of America. 2017; 114:11663-11668.

15. Li L, Cheng S, Gu J. SARS infection among health care workers in Beijing, China. JAMA. 2003;290(20):26622663.

16. Tan BYQ, Chew NWS, Lee GKH et al. Psychological Impact of the COVID-19 Pandemic on Health Care Workers in Singapore. Ann Intern Med 2020. 
17. Mowbray H. In Beijing, coronavirus 2019-nCoV has created a siege mentality. British Medical Journal, $2020 ; 368$. [Epub ahead of print]

18. Purgato M, Gastaldon C, Papola D, van Ommeren M, Barbui C, Tol WA. Psychological therapies for the treatment of mental disorders in low- and middle-income countries affected by humanitarian crises. Cochrane Database Syst Rev. 2018;7:CD011849.

19. Shultz JM, Cooper JL, Baingana F et al. The Role of Fear-Related Behaviors in the 2013-2016 West Africa Ebola Virus Disease Outbreak. Curr Psychiatry Rep. 2016;18(11):104.

20. Druss BG. Addressing the COVID-19 Pandemic in Populations With Serious Mental Illness. JAMA Psychiatry 2020.

21. Wallace CL, Wladkowski SP, Gibson A, White P. Grief During the COVID-19 Pandemic: Considerations for Palliative Care Providers. J Pain Symptom Manage. 2020. pii: S0885-3924(20)30207-4.

22. Galea S, Merchant RM, Lurie N. The Mental Health Consequences of COVID-19 and Physical Distancing: The Need for Prevention and Early Intervention. JAMA Intern Med 2020.

23. Ransing R, Adiukwu F, Pereira-Sanchez V et al. Mental Health Interventions during the COVID-19 Pandemic: A Conceptual Framework by Early Career Psychiatrists. Asian J Psychiatr. 2020;51:102085.

24. Kim SW, Su KP. Using psychoneuroimmunity against COVID-19. Brain Behav Immun. 2020; S08891591(20)30391-3.

25. Zhu S, Wu Y, Zhu CY et al. The immediate mental health impacts of the COVID-19 pandemic among people with or without quarantine managements. Brain Behav Immun. 2020;S0889-1591(20)30601-2.

26. Evans J, Repper J. Employment, social inclusion and mental health. Journal of Psychiatric and Mental Health Nursing. 2000;7:15-24.

27. Brooks SK, Webster RK, Smith LE et al. The psychological impact of quarantine and how to reduce it: rapid review of the evidence. Lancet 2020; 395:912.

28. Pfefferbaum B, North CS. Mental Health and the Covid-19 Pandemic. N Engl J Med 2020.

29. Reger MA, Stanley IH, Joiner TE. Suicide Mortality and Coronavirus Disease 2019-A Perfect Storm? JAMA Psychiatry 2020.

\section{Correspondence:}

Irina Mihaela Abdulan,

“Grigore T Popa" University of Medicine and Pharmacy - Iasi, Romania, Faculty of Medicine, Department of Medical Specialties (I), University street nr 16, Iasi, Romania; Clinical Rehabilitation Hospital - Cardiovascular Rehabilitation Clinic, Pantelimon Halipa street nr 14, Iasi, Romania, irina.abdulan@yahoo.com 



\title{
The impact of COVID-19 infection of patients with autoimmune blistering diseases of the skin
}

\author{
Ioana-Adriana Popescu, Dumitrița-Lenuța Nichitean, \\ Dan Vâță, Laura Stătescu, Ioana Alina Grăjdeanu, \\ Elena Porumb-Andrese, Laura Gheucă Solovăstru
}

Ioana-Adriana Popescu - M.D., Ph. D. student, Assistant Professor, Department of Dermatology "Grigore T. Popa" University of Medicine and Pharmacy Iași, Romania

Dumitrița-Lenuța Nichitean - M.D., Dermatology Clinic County Emergency Hospital "St. Spiridon" Iași, Romania

Dan Vâță - M.D., Ph. D., Lecturer, Department of Dermatology “Grigore T. Popa” University of Medicine and Pharmacy Iași, Romania

Laura Stătescu - M.D., Ph. D., Lecturer, Department of Dermatology "Grigore T. Popa" University of Medicine and Pharmacy Iași, Romania

Ioana Alina Grăjdeanu - M.D., Ph. D student, Assistant Professor, Department of Dermatology "Grigore T. Popa" University of Medicine and Pharmacy Iași, Romania

Elena Porumb-Andrese - M.D., Ph. D., Assistant Professor, Department of Dermatology “Grigore T. Popa" University of Medicine and Pharmacy Iași, Romania

Laura Gheucă Solovăstru - M.D., Ph. D., Professor, Department of Dermatology “Grigore T. Popa" University of Medicine and Pharmacy Iași, Romania

\begin{abstract}
Nowadays, it is an uncertain time for the whole world, firmly under the grip of a tiny virus, thought to have originated in China. The disease caused by the SARS-CoV-2 virus was termed Coronavirus disease/COVID-19. As dermatologists, we are impacted in multiple ways in the COVID-19 pandemic and have a significant role to play. Autoimmune blistering diseases (AIBD) are a miscellaneous group of disorders associated with autoantibodies directed against distinct desmosomal structural proteins, hemidesmosome structural proteins, or epidermal/tissue transglutaminase. AIBD patients need continuous hospital monitoring, and during this period affected by the pandemic with COVID-19, their physical and mental condition suffered. The cutaneous damage such as bullae formation, pain, itch, and associated
\end{abstract}


functional limitations have a psycho-emotional impact on patients and can severely affect the patient's quality of life.

\section{KEYWORDS:}

Autoimmune bullous diseases, Covid-19 infection, morbidity, quality of life.

\section{INTRODUCTION}

Nowadays, it is an uncertain time for the whole world, firmly under the grip of a tiny virus, thought to have originated in China. A novel enveloped RNA beta corona virus, it was named- Severe acute respiratory syndrome coronavirus due to its structural similarity to the virus causing severe acute respiratory syndrome (SARS). The disease caused by the SARS-CoV-2 virus was termed Coronavirus disease/COVID-19 $(1,2)$.

In December 2019 COVID-19 was reported for the first time, it has spread far and wide across the globe to cause unforeseen health hazards and critical challenges impacting the entire spectrum of life. As doctors, we have a responsibility to support measures which mitigate the spread of severe acute respiratory syndrome coronavirus 2 (SARS-CoV-2), but at the same time, to provide essential medical care to our patients. Cutaneous bullous pathology poses a unique set of challenges ( 1 , 2).

As dermatologists, we are impacted in multiple ways in the COVID-19 pandemic and have a significant role to play. For example, we observe an important psychoemotional impact to patients with autoimmune bullous diseases. Their skin is fragile, the skin barrier has discontinuities and then it would be more at risk to developing Covid 19 infections.

Autoimmune blistering diseases (AIBD) are a miscellaneous group of disorders associated with autoantibodies directed against distinct desmosomal structural proteins, hemidesmosome structural proteins, or epidermal/tissue transglutaminase. These autoantibodies result in the loss of cell adhesion within the epidermis and between the epidermis and dermis, resulting in the cleavage and formation of blisters or erosions.
AIBD occurring in the elderly include bullous pemphigoid (BP), mucous membrane pemphigoid (MMP), epidermolysis bullosa aquisita (EBA), paraneoplastic pemphigus (PNP), pemphigus vulgaris (PV), pemphigus foliaceus (PF), linear IgA dermatosis (LIAD) and dermatitis herpetiformis (DH) (3).

The most common bullous autoimmune dermatosis, bullous pemphigoid is characterized by marked clinical variability and intense pruritus (4). The second most common bullous autoimmune dermatosis, pemphigus vulgaris, typically presents with oral erosions as the predominant and frequently - initial symptom. In Bullous Pemphigoid (BP) subepidermal blistering is induced by autoantibodies against hemidesmosomal structural proteins $(5,6,7)$. Pemphigus vulgaris (PV) is a chronic autoimmune bullous dermatosis that results from the production of autoantibodies against desmogleins 1 and 3 (8).

\section{SKIN CARE IN AIBD}

On the fragility of the skin, it is emphasized that any pressure in apparently normal skin, especially near the injury induces epidermal detachment. Although the individual may present large areas of healthy skin, it is noteworthy that all the skin is vulnerable to further injury (9).

Thus, any pressure on the skin, even during routine nursing care, can increase the injured area, and this a fact confirmed by the positive Nikolski sign (+). This signal is characterized by partial or complete detachment of the epidermis, which is made through the finger pressure on perilesional skin which is found in pemphigus vulgaris. Disruption of bullous lesions gives rise to eroded, exulcerated and exudative lesions, with possible loss of fluid and protein, a group of symptoms that 


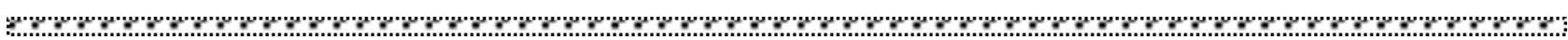

predisposes to infection, dehydration, anemia and deep malnutrition $(9,10)$.

In this period, skin lesions have been reported following frequent hand washing and the use of hand sanitizers. Moreover, skin problems related to personal protective equipment was reported. Aggravation of previous dermatitis has been noted in the wearers and also skin lesions ranging from contact and pressure. Contact dermatitis and hand dermatitis in atopic patients can be a rising concern. The dermatological recommendation is to repeat use of thick emollients and barrier creams to mitigate the damage of the skin.

AIBD patients need continuous hospital monitoring, and during this period affected by the pandemic with COVID-19, their physical and mental condition suffered.

Facing the interference of skin condition in the physical, mental, environmental and social spheres makes the identification of the diagnosis "impaired comfort" understandable. Regarding this diagnosis, there is the pain caused by lack of skin integrity and the difficulty presented by the patients to rest in bed, both interfering negatively in sleep patterns. Moreover, it is necessary to mention the discomfort caused by the patients' exposure to the stigmatizing eyes of society, given the impossibility of hiding a problem that is imprinted on your skin. These factors make the evaluation of that patient in their entirety essential in order to identify the problems and implementation of nursing actions that promote comfort (11).

Always adjuvant to systemic treatment, topical treatment of lesions aims to reduce pain and prevent secondary infection. It is usually performed with corticosteroid and/or antibiotic creams $(12,13)$.

Individual case reports have described a response to topical treatment with the calcineurin inhibitor, tacrolimus. It has mainly been used for localized and limited generalized disease. The use of topical tacrolimus is limited by local irritation and its price compared with topical steroids. It may be useful as an alternative in localized and limited disease without the disadvantage of causing skin atrophy $(14,15)$.

Due to their low immunity, the risk of develop COVID-19 infection increases. In addition, the mandatory wearing of protective equipment favors the appearance of new lesions, with the possibility of bacterial infection.

Blisters should generally be left intact if possible as this may help prevent secondary bacterial infection. When they are particularly large or in sites where they are troublesome or interfere with function, such as the sole of the foot, blisters may be pierced with a sterile needle releasing the fluid, but leaving the blister roof in place, as it forms a natural dressing. If the blister is already broken remove only the fluttering skin (16-18).

If there are extensive areas of erosion and open raw areas, antiseptics such as potassium permanganate as a bath or soaks, or antiseptic-containing bath oils may be used for a few days to dry the lesions and prevent infection (16).

In cases of extensive erosive lesions, the latter may be covered by bandages using different types of dressings, preferably nonadherent, to reduce bacterial superinfection and pain, as well as to promote healing (16-18).

Patients should be monitored for drug sideeffects and to ensure that symptoms are controlled to their satisfaction without excessive doses of topical or systemic treatment. Occasional itching or lesions (if acceptable to the patient) indicates that they are not being overtreated $(16,19)$.

\section{CONCLUSIONS}

It is a difficult period for all of us, especially for patients with autoimmune blistering diseases, due to restricted access to qualified medical care, the obligation to wear protective equipment, with very important implications on psycho-social factors. 
The cutaneous damage such as bullae formation, pain, itch, and associated functional limitations have a psycho-emotional impact on patients and can severely affect the patient's quality of life. These symptoms, by their despicable character, can have a significant burden on social function, independent of clinical severity.

It is important to educate patients and their families about the risks of developing psychiatric side effects during corticosteroid therapy and the need to monitor these patients. Moreover, during this period they must keep in touch with their attending physician to safely continue treatment and to avoid any side effects.

\section{ACKNOWLEDGEMENTS AND DISCLOSURES}

The authors have no potential conflict of interests to disclose.

\section{REFERENCES}

1. Jagadeesan S, Sarkar R. COVID-19 and the dermatologist: finding calm in the chaos. Pigment Int. http://www.pigmentinternational.com/preprintarticle.asp?id=282601

2. WHO Coronavirus disease. https://www.who.int/emergencies/diseases/novel-coronavirus-2019

3. Lakos J. I, Jerkovic G.S, Branka M. Blistering Diseases in the Mature Patient. Clinics in Dermatology. 2017; doi: 10.1016/j.clindermatol.2017.10.014.

4. Hofmann S.C, Juratli H.A., Eming R. 2018. Deutsche Dermatologische Gesellschaft (DDG). Published by John Wiley and Sons Ltd.

5. Mutasim D.F. Autoimmune bullous dermatoses in the elderly: an update on pathophysiology, diagnosis and management. Drugs Aging 2010; 27: 1-19.

6. Sticherling M, Erfurt-Berge C. Autoimmune blisterig diseases of the skin. Autoimmunity Reviews. 2012; 226230.

7. Schmidt E, Zillikens D. Research in practice: diagnosis of subepidermal autoimmune bullous disorders. J Dtsch Dermatol Ges. 2009; 7:296-300.

8. Pollmann R, Schmidt T, Eming R, Hertl M. Pemphigus: a complehensive review in pathogenesis, cinical presentation and novel therapeutic approaches. Clin Rev Allergy Immunol 2018; 54: 1-25.

9. Cunha PR, Barraviera SR. Autoimmune bullous dermatoses. An Bras Dermatol. 2009;84(2):111-24.

10. Brandão ES, Santos I, Lanzillotti RS, Ferreira AM. Et al. Rev. Latino-Am. Enfermagem 2016; 24 : e2766.

11. Santos I, Caldas CP, Gauthier J. at al. Caring for the whole person: the contributions of aesthetics/sociopoetics perspectives to the field of nursing. Rev Enferm UERJ. 2012;20(1):49.

12. Porro AM, Seque CA, Ferreira MCC. At al. Pemphigus vulgaris. An Bras Dermatol. 2019;94(3):264-78.

13. Gach JE, Ilchyshyn A. Beneficial effects of topical tacrolimus on recalcitrant erosions of pemphigus vulgaris. Clin Exp Dermatol. 2004; 29:271-2.

14. Chu J, Bradley M, Marinkovich MP. Topical tacrolimus is a useful adjunctive therapy for bullous pemphigoid. Arch Dermatol 2003; 139:813-15.

15. Calcaterra R, Carducci M, Franco G et al. Topical tacrolimus treatment for localized pretibial bullous pemphigoid. J Eur Acad Dermatol Venereol 2009; 23:177-9.

16. Venning V.A, Taghipour K., Mohd Mustapa M.F.et al. Guidelines for the management of bullous pemphigoid 2012, V.A. Venning et al. British Association of Dermatologists 2012;167:1200-1214.

17. Le Roux-Villet C, Prost-Squarcioni C, Oro S et al. Role of the nurse in care of bullous pemphigoid. Rev Infirm 2010; 160:38-40.

18. Feliciani C, Joly P, Jonkman M.F. et al. Management of bullous pemphigoid: the European Dermatology Forum consensus in collaboration with the European Academy of Dermatology and Venereology. British Journal of Dermatology 2015; 172:867-877.

19. Gheucă Solovăstru L, Vâță D, Stătescu L, Andrese E. - The pshychiatric burden of chronic dermatological diseases. Bulletin of Integrative Pshychiatry. 2014; 3(62): 31-41.

\section{Correspondence:}

Dan Vâță, MD,

"Grigore T. Popa" University of Medicine and Pharmacy, Faculty of Medicine, Dermatology Department, Str. Universitatii nr. 16, 700115, Iasi, Romania, danvata@yahoo.com 


\title{
Is cardiovascular risk factor prevention necessary? The influence of diabetes mellitus in the development of Alzheimer's dementia
}

\author{
Ioana A. Sandu, Anca I. Pîslaru, Adina C. Ilie, Ramona Ștefăniu, \\ Călina A. Sandu, Anisia Alexa, Ioana D. Alexa, Cristinel Ștefănescu
}

Ioana A. Sandu - MD, PhD student, Assistant professor , "Grigore T. Popa" University of Medicine and Pharmacy Iași, Romania

Anca I. Pîslaru - MD, PhD, Lecturer, "Grigore T. Popa" University of Medicine and Pharmacy Iași, Romania

Adina C. Ilie - MD, PhD, Lecturer, “Grigore T. Popa” University of Medicine and Pharmacy Iași, Romania

Ramona Ștefăniu - MD, PhD student, Assistant professor , "Grigore T. Popa" University of Medicine and Pharmacy Iași, Romania

Călina A. Sandu - resident physician, Saint Spiridon Hospital, Iași

Anisia Alexa - MD, PhD, Assistant professor , "Grigore T. Popa" University of Medicine and Pharmacy Iași, Romania

Ioana D. Alexa - MD, PhD, senior geriatrics and gerontology, professor, "Grigore T. Popa" University of Medicine and Pharmacy Iași, Romania

Cristinel Ștefănescu - MD, PhD, senior psychiatrist, professor, “Grigore T. Popa” University of Medicine and Pharmacy Iași, Romania

\begin{abstract}
Alzheimer's disease (AD) is the most common cause of dementia in the elderly, causing major progressive deficits in cognitive function and memory. Currently, over 35 million people worldwide are affected by this disease. Hyperglycemia is a well-known risk factor for the development of metabolic diseases in adults, but recent studies have shown that it is also closely linked to the development of cognitive impairment and dementia. Moreover, chronic exposure to hyperglycemia can affect cognitive function and other mental diseases as well. This is worrying considering that over 176 million people are currently diagnosed with diabetes, and estimated to reach 366 million by 2030 . The aim of the study is to evaluate the relationship between the presence of diabetes and other cardiovascular risk factors and the risk of developing AD.
\end{abstract}

KEYWORDS:

Diabetes, dementia, Alzheimer disease, senior patients. 


\section{INTRODUCTION}

Dementia is an "umbrella" term that encompasses many neurodegenerative disorders, including Alzheimer's disease (AD), which has an insidious onset and a slow progression. The prevalence is increasing, the global costs estimated by 2015 were US $\$ 818$ billion annually, and they will expand to 2 trillion by 2030. Clinical diagnosis can be often difficult, and this is the reason why there were designed new methods, which were introduced in the last decade in order to facilitate the early diagnosis and, more importantly, prevention of AD (1). The application of these methods is constantly increasing, partly due to the high degree of awareness of cognitive symptoms and the relevance of early detection and diagnosis, especially when more effective drugs are available to treat this pathology. Currently, only symptomatic treatment can be prescribed, and the focus should be on potential prophylactic strategies (2).

Obesity is a major risk factor for $\mathrm{AD}$, because it induces adipokine breakdown, which consists of the release of pro-inflammatory adipokines and the decrease of antiinflammatory adipokines among other processes. On the other hand, obesity is one of the most important modifiable risk factors for the prevention of Type 2 Diabetes. Moreover, it has been shown that being overweight is associated with an increased prevalence of high blood pressure, dyslipidemia and is associated with an increased risk of mortality, especially due to cardiovascular diseases $(3,4)$. This is due to changes in lifestyle, for example, low levels of physical activity, an unbalanced diet and excessive nutrition, leading to processes of inflammatory and oxidative stress, thus altering the metabolic pathways required for homeostasis $(5,6)$.

Strategies for the prevention of $\mathrm{AD}$ through non-pharmacological treatments are associated with lifestyle interventions such as exercise, mental challenges and socialization, as well as caloric restriction and a healthy diet $(3,6)$ (see fig 1$)$.

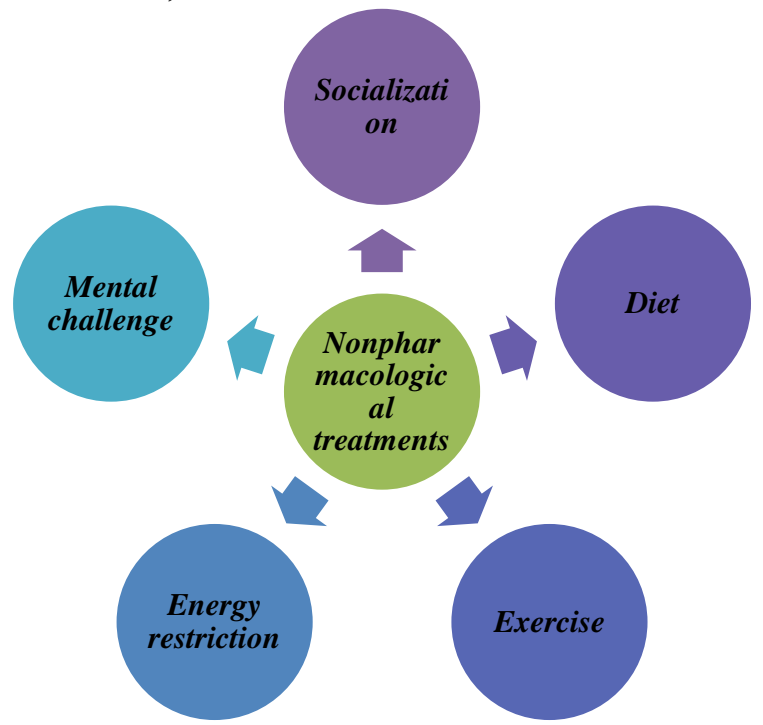

Fig.1 Non-pharmacological treatments strategies for the prevention of AD

The beneficial effects of physical activity are well known; however, beyond the general recommendations available for health promotion, regarding the type, frequency, intensity and duration of physical activity that could protect against $\mathrm{AD}$, specific practical recommendations are not yet well known. However, at present, physical activities that have additional components of social and cognitive stimulation are considered to be the most effective. $(7,8,9)$

$\mathrm{AD}$ is a very important health problem that requires screening to monitor cardiovascular risk factors, so that prevention strategies can be implemented in order to minimize the risk of its development (10). 
Bulletin of Integrative Psychiatry O New Series OSeptember 2020 ○ Year XXVI ONo. 3 (86)/41

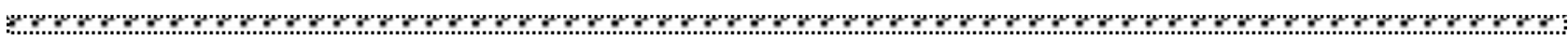
In the proposed study, our objectives were to evaluate cardiovascular risk factors among the adult population and the risk of developing Alzheimer's Dementia after 20 years, and the influence of pharmacological and nonpharmacological treatment of cardiovascular risk factors has over the risk of developing AD.

\section{METHODOLOGY}

We present partial results of a prospective study conducted between January-August 2019. The study group consisted of 40 patients, aged between 40-65 years, hospitalized in the Internal Medicine Ambulatory and the Hospital Geriatrics Clinic "Dr. C. I. Parhon ", Iasi.

The realized balance included the following:

- Biological exploration

- Evaluation of cardiovascular risk factors

- Comprehensive geriatric evaluation

- Assessment of the risk of developing Alzheimer's dementia - CAIDE score (tab.I)

All subjects signed an informed consent questionnaire and had the opportunity to withdraw at any time during the study period.

Tabel I CAIDE score

\begin{tabular}{|c|c|c|c|}
\hline \multicolumn{4}{|c|}{$\begin{array}{lcc}\text { CAIDE } & \text { SCORE: } & \text { PROBABILITY } \\
\text { DEVELOPING DEMENTIA IN } 20 \text { YEARS }\end{array}$} \\
\hline \multicolumn{2}{|c|}{ RISK FACTORS } & \multicolumn{2}{|c|}{ POINTS } \\
\hline$<47$ & 0 & & \\
\hline $47-53$ & 3 & & \\
\hline$>53$ & 4 & & \\
\hline$>10$ years & 0 & & \\
\hline 7-9 years & 2 & & \\
\hline$<7$ & 3 & \multirow{2}{*}{$\begin{array}{l}\text { Total } \\
\text { score }\end{array}$} & \multirow{2}{*}{$\begin{array}{l}\text { Dementia } \\
\text { risk }\end{array}$} \\
\hline Female & 0 & & \\
\hline Male & 1 & $0-5$ & $1 \%$ \\
\hline$<140 \mathrm{mmHg}$ & 0 & $6-7$ & $1,9 \%$ \\
\hline$>140 \mathrm{mmHg}$ & 2 & $8-9$ & $4,2 \%$ \\
\hline$<30 \mathrm{Kg} / \mathrm{m} 2$ & 0 & $10-11$ & $7,4 \%$ \\
\hline$>30 \mathrm{Kg} / \mathrm{m} 2$ & 2 & $12-15$ & $16,4 \%$ \\
\hline$\overline{<<6,5 \mathrm{mmol} / 1}$ & 0 & & \\
\hline$>6,5 \mathrm{mmol} / 1$ & 2 & & \\
\hline Yes & 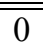 & & \\
\hline No & 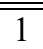 & & \\
\hline
\end{tabular}

\section{RESULTS}

The age of the patients was between 40-65 years, which is one of the mandatory inclusion criteria in this study. The gender distribution of patients was as follows: (fig.2)

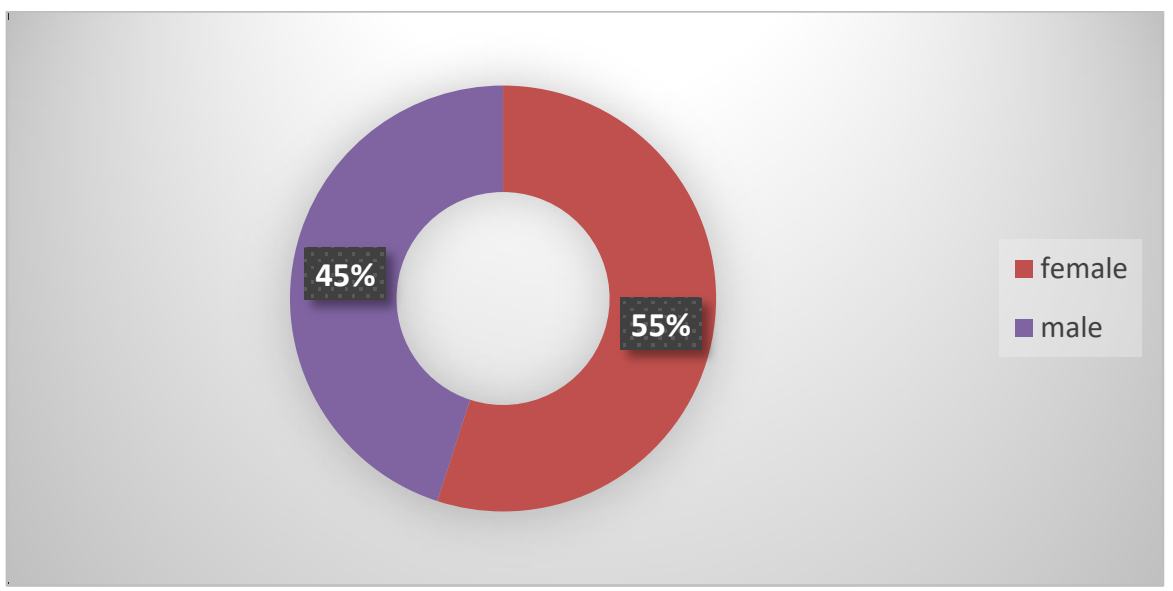

Fig. 2 Distribution of patients by sex.

The incidence of Type 2 Diabetes in the group of patients studied showed that the percentage in women is $20 \%$ higher than in men (Fig. 3) 


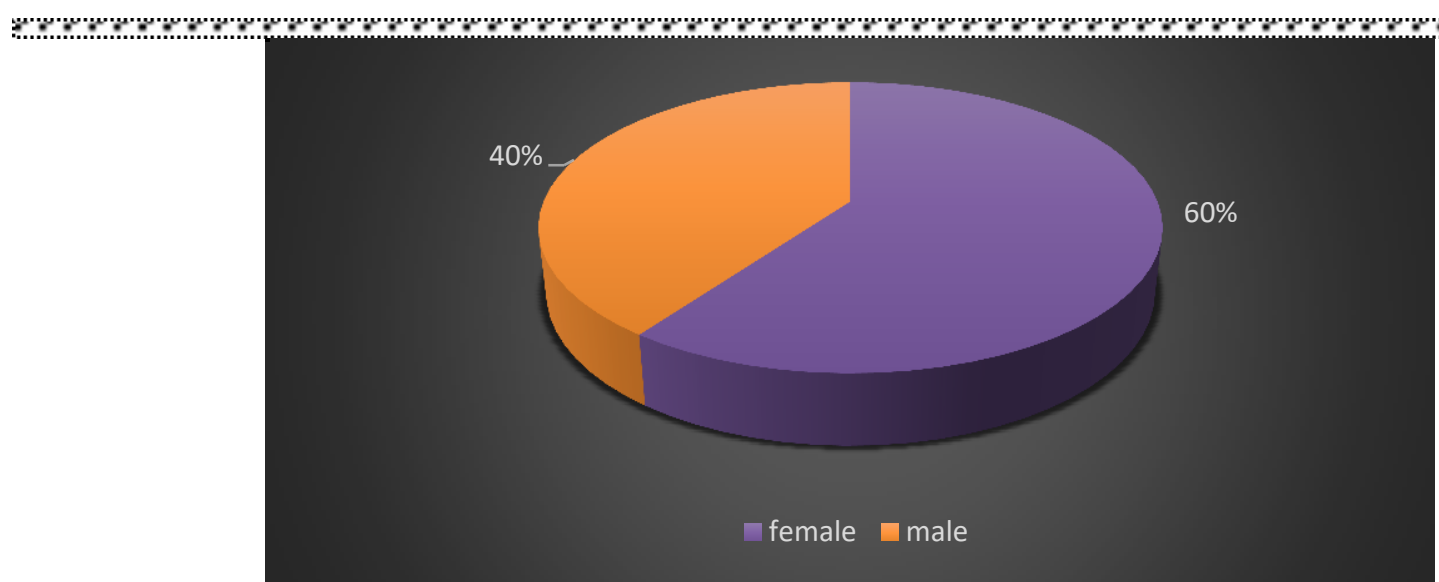

Fig 3. The incidence of type 2 diabetes mellitus by sex.

The BMI analysis in the study showed that out of the total number of patients, the highest weight is of overweight patients (38.9\%) and those with grade I obesity (27.8\%) (Fig. 4):

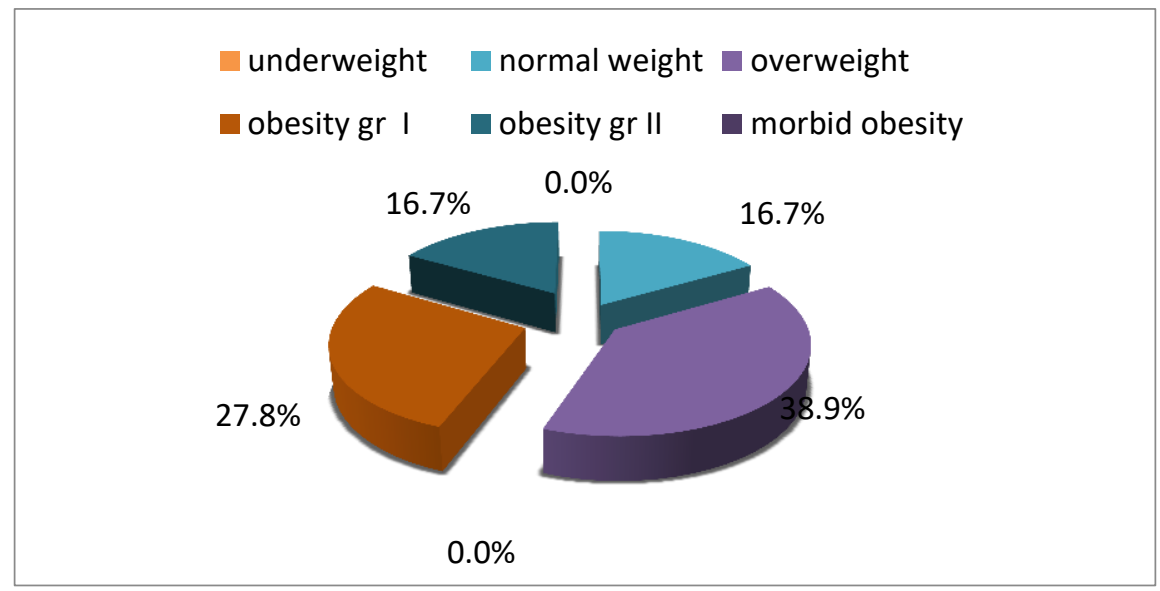

Fig. 4 BMI analysis in the studied group.

It was also observed that most patients: $75 \%$

have a low level of physical activity (fig. 5):

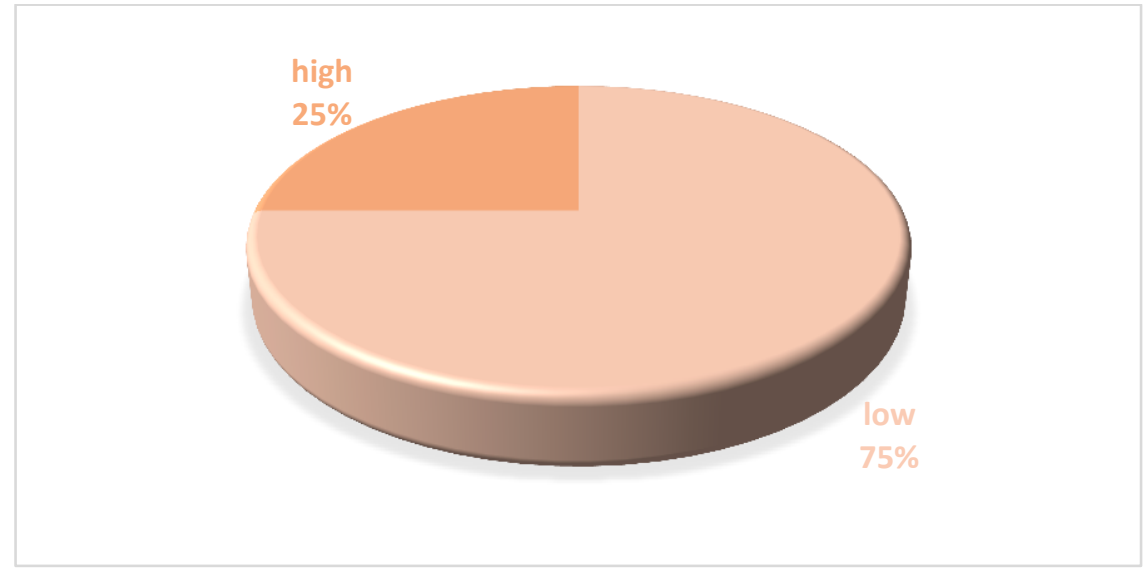

Fig. 5 The incidence of physical activity in the studied group.

Using the CAIDE score, we identified (fig. 6):

- Regarding the average risk of developing $\mathrm{AD}$, it can be seen that women are the ones with the highest incidence.
- A higher percentage of male patients in the study group have an increased risk of developing Alzheimer's dementia compared to female patients. 


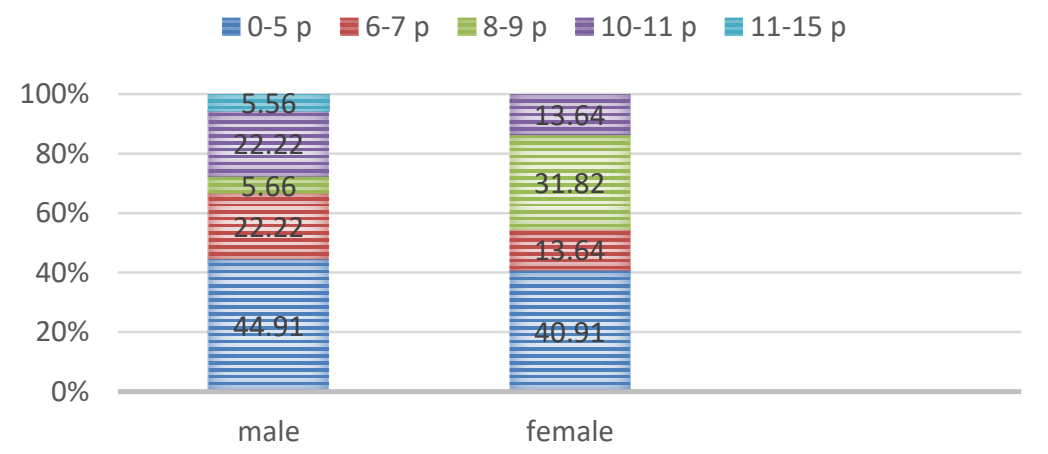

Fig. 6 The incidence of the risk developing Alzheimer's using CAIDE score in the studied group.

\section{DISCUSSIONS}

$\mathrm{AD}$ is a multifactorial disease and the combination of two or more nonpharmacological treatments for prevention is extremely important, of course in combination with drug therapy $(3,6)$.

Socialization is also an important component for human mental and physical development, and its lack induces loneliness by increasing the risk of: depression, obesity, diabetes, hypertension, and $\mathrm{AD}(4,6)$.

The risk of developing $\mathrm{AD}$ should be considered from early ages, to avoid risk factors, and for the elderly, it is important to improve treatments by adopting a healthier lifestyle and dietary changes; in order to strengthen prevention (10)

There is a lot of obesity type diabetes, that is closely related to the factors that promote the evolution towards AD (lack of physical activity, stress, lack of socialization-witch are the main preventive elements in $\mathrm{AD}$ ).

Our study demonstrates once again that there is a close relationship between the presence of cardiovascular risk factors and the increased risk of developing $\mathrm{AD}$. Also, the lack of physical activity and obesity are closely linked to the presence of DM and the increased risk of developing AD.

\section{CONCLUSIONS}

Given that there is currently no known remedy for $\mathrm{AD}$, further experimental research exploring preventive strategies is clearly needed. AD research needs to be further developed to propose new molecules for therapy; but what is most important for the purpose of developing prevention and screening measures.

Evidence suggests that diet and activity changes that lead to weight loss are especially effective in reducing diabetes risk. Preventing diabetes or managing it successfully helps avoiding cardiovascular complications and decreasing the risk of developing mild cognitive impairment and AD.

The multidisciplinary approach of $\mathrm{AD}$ for dementia prevention also seems more promising in comparison with the traditional, unidisciplinary approach.

\section{ACKNOWLEDGEMENTS AND DISCLOSURES}

The authors have no potential conflict of interests to disclose.

\section{REFERENCES}

1. Lee, H. J., Seo, H. I., Cha, H. Y., Yang, Y. J., Kwon, S. H., Yang, S. J. Diabetes and Alzheimer's Disease: Mechanisms and Nutritional Aspects. Clinical nutrition research.2018; 7(4): 229-240.

2. Ugeskr, L. Alzheimer's disease. 2017;179(12): 505-508.

3. Nguyen, N.T., Nguyen, XM.T., Lane, J. et al. Relationship Between Obesity and Diabetes in a US Adult Population: 
4. Qizilbash N., Gregson J., Johnson M. E. et al. BMI and risk of dementia in two million people over two decades: a retrospec- tive cohort study, The Lancet Diabetes and Endocrinology.2015;3(6):431-436.

5. G. Verdile, K. N. Keane, V. F. Cruzat et al. Inflamma- tion and oxidative stress: the molecular connectivity between insulin resistance, obesity, and Alzheimer's disease. Mediators of Inflammation. 2015.

6. Mendiola-Precoma, J., Berumen, L. C.,K. Padilla, Garcia-Alcocer, G. Therapies for Prevention and Treatment of Alzheimer's Disease, BioMed Research International. 2016.

7. R. Stephen, K. Hongisto, A. Solomon, E. Lönnroos. Physical Activity and Alzheimer's Disease: A Systematic Review, The Journals of Gerontology: Series A. 2017;72(6):733-739.

8. Brini S., Sohrabi H.R., Peiffer J.J. et al . Physical Activity in Preventing Alzheimer's Disease and Cognitive Decline: A Narrative Review. Sports Med.2018;48:29-44.

9. R. F. de Bruijn, Ikram M. A. Cardiovascular risk factors and future risk of Alzheimer's disease, BMC Medicine. 2014;12(1):1-9.

10. JL Etnier, WB Karper, JD Labban, AT Piepmeier, CH Shih, WN Dudley et al.The Physical Activity and Alzheimer's Disease (PAAD) Study: Cognitive outcomes, Annals of Behavioral Medicine. 2018;52(2):175-185.

\section{Correspondence:}

Anisia Alexa,

MD, PhD, Assistant professor , "Grigore T. Popa” University of Medicine and Pharmacy Iași, Romania, Universității Street nr.16, Iași, Romania, alexa_anisia@yahoo.com

Submission: 2 aug 2020

Acceptance: 4 sep 2020 


\title{
Non-drug modalities of managing anxiety in dental office
}

\author{
Georgiana Macovei, Alexandra Boloș, Magda Ecaterina Antohe, \\ Gente Camille, Antonela Maria Beldiman
}

Georgiana Macovei - lecturer, PhD, "Grigore T. Popa" University of Medicine and Pharmacy Iași, Romania, Faculty of Dentistry, Discipline of Orodental Diagnostic and Gerontostomatology Alexandra Boloș - MD, PhD, lecturer "Grigore T. Popa" University of Medicine and Pharmacy Iași, Romania, Faculty of Medicine, Discipline of Psychiatry

Magda Ecaterina Antohe - PhD, professor, "Grigore T. Popa University of Medicine and Pharmacy Iași, Romania, Faculty of Dental Medicine, Department of Implantology, Removable Dentures, Technology, Discipline of Partial Removable Prostheses

Gente Camille - dentist, Paris, France

Antonela Maria Beldiman - PhD, lecturer, "Grigore T. Popa" University of Medicine and Pharmacy Iași, Romania, Faculty of Dentistry, Discipline of Prosthesis Technology

\begin{abstract}
Anxiety disorders are a group of psychiatric disorders whose symptoms include excessive anxiety, a feeling of fear, worry and avoidance behaviors. Dental anxiety may have a negative impact on a person's life. Physiological aspects of dental anxiety are fright response and feelings of exhaustion after dental appointments, while behavioral aspects are represented by dental avoidance. The practitioner must undertake psychological support for the patient in a dental office where colors and music are used to create a welcoming, calm space that inspires relaxation, benefiting from a pleasant light adapted to each work or waiting space. The dental office is a place with a strong anxiety-inducing potential, that's why the first step of the practitioner is to recognize the patient's fear as existing and not to deny it, or to underestimate it and to find, together with the patient all the necessary modalities to cope with it.
\end{abstract}

\section{KEYWORDS}

Anxiety, dental office, management. 


\section{INTRODUCTION}

Anxiety disorders are a group of psychiatric disorders that bring together several clinical entities that are now well characterized with anxiety as the common symptom. Fear is a primary emotion, it is universal and is felt by all living beings. Fear allows the subject to protect himself and to escape from a potential danger, it functions as a warning signal. But this fear, which at first glance may seem salutary in the face of a threat, changes pathologically when it is triggered in inappropriate circumstances.(1) On the other hand, stress is defined as the body response following any request or solicitation exerted on the body. The anxiety is expressed by a painful feeling of psychic alert and somatic mobilization in the face of an undetermined threat or danger and manifested by characteristic neurovegetative symptoms (spasms, sweating, dyspnea, increased heart rate, dizziness, etc.) and phobia is a "distressing and unjustified fear of a situation, an object or the performance of an action". (2) The origins of the fear of the dentist are multifactorial, which makes the management even more complex, and requires individual adaptation on the part of the practitioner. Initially, this fear develops, often during childhood by direct conditioning following an experience, dental or medical, generally negative or indirect, by the influence of a third person. Some studies show a strong correlation between mother's anxiety and that of her child.

Alain Amzalag has stated some origins of the fear of the dentist: transgenerational fears with received ideas and transmitted by parents; fears acquired through lived experiences, which are found in the unconscious, rejection of access to the intimate sphere, with a position of submission, of no control; fear of invasion of intimate space; fear of getting infections, diseases (especially nosocomial); fear of scams. (3) As mechanisms of fear we can say that it is the result of a chain reaction. (4) The essential element of its brain pathway is the cerebral amygdala, a bilateral brain structure that is located near the hippocampus in the frontal (antero-inferior) part of the temporal lobe. The main role of the amygdala is to analyze and assess the positive or negative connotation of sensations and to initiate a reaction accordingly. It acts as an alarm system. The amygdala registers information from the thalamus and then produces preprogrammed emotional and motor responses to deal with the danger such as: immobilization, change in blood pressure and heart rate, or release of hormones. The other region of the brain involved in the fear mechanism is the hippocampus. The major role of the hippocampus is the storage and recall of memories. We can therefore say that the hippocampus informs the amygdala, it gives it information in reference to past experiences, on the seriousness of the situation as well as on the importance of reacting to it.

Dental anxiety may have a negative impact on a person's life. According to some studies, physiological aspects are fright response and feelings of exhaustion after dental appointments, while behavioral aspects are represented by dental avoidance. It is well known that anxious patients avoid dental treatment, either by failing to appear for their dental appointments or by delaying dental visits for long periods of time (5). The interaction between anxiety and dental pain suggests that people who respond fearfully to pain are at an increased risk of ending up in a vicious cycle of anxiety. If this cycle is not broken, a severe form of dental fear might develop known as dental phobia. This phobia is characterized by marked and persistent anxiety in relation either to clear discernable 
s

situations (hand piece sound, needle, smells) or to the dental situation in general $(6,7,8)$.

The assessment of the patient's level of anxiety is based on observation of the patient, his medical questionnaire and assessment through scales. There are many scales for assessing anxiety like The Corah Scale (Dental Anxiety Scale), The Kleinknecht Test, Hamilton Anxiety Rating Scale: HAMA, child specific scales like The Venham Picture Scale, Child Fear Survey Schedule Dental Subscale (CFSS_DS ), The Facial Image Scale. Each scale assesses a different aspect of the anxiety situation and evolution. To choose the appropriate test, the clinician must take into account several criteria as the age of the patients (children, adults), patient choice and individual characteristics. The abundance of these tools can sometimes inconvenience the practitioner in comparing their results. The choice must therefore be made according to the objectives and the information sought.

Non-drug modality that we can use in every day practice to reduce dental anxiety is the ergonomics of the practice, the effect of colors and music. In the case of the dental office, ergonomics concerns the spaces dedicated to the patient population and the medical team as well as the arrangement of equipment and devices. When arranging the rooms of a dental office, all the structures that make it up must be studied with great care. The configuration of the cabinet must take into account the furniture and their locations, the shape of the rooms and the corridors. In general, the practice is divided into two areas: the reception and waiting area for patients, and the so-called clinical area. The first contact with the patient is through the reception. The reception for the patient is therefore a reflection of the image of the dental practice. $(9,10)$
Chromotherapy and light therapy, the roots of the therapeutic effects of color can be traced way back to the ancient times. Avicenna, in his Canon of Medicine, made clear the vital importance of color in both diagnosis and treatment. He had developed a chart that related color to temperament and the physical condition of the body. According to his findings red color had an energetic effect, blue, violet or white had a calming effect on blood circulation, and yellow reduced pain and inflammation. $\mathrm{He}$ also considerate the possible dangers of color in treatment. (11)

Color is the property of light, which consists of many different electromagnetic waves of energy. When light falls upon the photoreceptor cells of the retina, it is converted into electric impulses, which then travel to the brain and trigger the release of hormones.(12) Chromotherapy is a method that treats psychological and physiological disorders by using colored light. Colors not only have an aesthetic effect, they have an influence on the physiological and psychological state. The effects felt can be expressed in several aspects: creation of a feeling of aggression or joy, a feeling of cold or hot (psychological effects) or for example, an excitation of the nervous system leading to changes in the heart rate, pulse or blood pressure (physiological effects). In order to avoid unconscious stress on the patient, due to the environment of the dental practice, the choice of colors in the different spaces is important. At the reception, colors that inspire interaction, dynamism and sociability, such as yellow, orange or turquoise, will be preferred. In the waiting room, shades will be chosen that inspire relaxation, such as blue or green, or rather warmer shades to create a cozier atmosphere. Within the treatment room, it will be preferable to choose light main colors, preferably, or blue or green. Light also plays a very important role in balancing the patient's 
r.\%\%

condition. First, take into account natural light, sunlight affects the patient's state of mind, and color changes throughout the day can influence heart rate and wakefulness. Then, artificial light is present in all the rooms of the practice and helps to contribute to the well-being of the patient and the practitioner, visual comfort must be established. In the treatment room, there are many sources and we must try to create a harmonious environment between the operating light, the ceiling light and the auxiliary lighting. Color is in relation with different situations and is reflecting different emotions, reactions and behaviors. In the dental environment, color preference could be a measure that reflects and emphasizes particular emotional states, fear and dental anxiety. Researchers claimed that specific color trigger specific emotional responses. We often relate to colors that are most pleasing at the time of interaction with them, as colors appeal emotionally and aesthetically. Specific colors usually elicit particular emotions, but the particular emotions involved are in not the same for each individual. The emotion triggered by a color depends on nationality, past experiences, and sometimes personal preferences. $(13,14)$

The complex relation between colors, light and human emotions indicate that the use of color interpretation and emotional association has the potential to become a useful tool in the pediatric dentist's armory for intervention and treatment.(15) Not only the colors used in the arrangement of the office, but the color of medical uniforms have an impact on anxious patients. There are studies that indicate that artistic activities, such as drawing in the waiting room have a positive effect on little anxious patients.(16) Completing the influence of colors on the perception of the environment, sounds can have a positive or negative impact on the well-being of the patient. The positive influence of music has long been known and despite a lack of scientific recognition, physicians and dentists do not hesitate to use it as a therapeutic tool.

Music therapy has the advantage of being easy to set up. First of all, in the waiting room it creates a pleasant atmosphere. But also in treatment rooms where it allows the patient to relax, masks stressful noises such as the noise of the turbine. Music then helps restore, or improve, a person's emotional balance. Music can also help fill conversation voids, when the practitioner is focused and the patient cannot speak, during periods of waiting that can create silent discomfort. Music therapy is like an exogenous cue that can be practiced in dentistry for anxious patients as it reduces anxiety, whereas having no effect on pain. Music therapy and attentive use of colors are an effective, complementary, nonpharmacological method contributing to the traditional clinical procedures that can make the dental visit more attractive and relaxed. (18)

Music's impact on our physiological and cognitive state is still in research. In a lot of studies, patients were asked to listen the prerecorded music through headphones for the duration of the dental procedure. Reactions to music are highly individual and context dependent. They vary with variables as personality, age, taste and cultural background. Generalities apply and certain patterns of musical structure may have predictable impacts on listeners, so it may be possible to predict and therefore prescribe music as a psychobiological approach regarding to dental anxiety. But we can say that for example, a piece of music might have a generally relaxing effect on most patients (due to its intrinsic musical structures), but 
the opposite effect on one patient who had first heard it in a disturbing event. (19)

Anyway to obtain a relationship conducive to success, the caregiver must detach himself from his purely technical approach. He must appear passionate, convinced and convincing while expressing empathy and kindness towards the patient. He must exhibit his human qualities more than his technical skills.

\section{CONCLUSIONS}

Anxiety produces an avoidance behavior that results in a large installation oral pathology, ascending to local and general complications. Due to a direct influence on oral health status, anxiety should be detected and treated properly, patients requiring a multidisciplinary approach.

The practitioner must adjust to his patient in order to offer him the solution that seems most appropriate to him. It is a complex relationship, which is too often wrongly approached with a mechanical issue, when it is important to maintain a social relationship with your patient. The goal is to obtain a practitioner-patient partnership in order to create a therapeutic alliance.

The dental office is a place with a strong anxiety-inducing potential, the first step of the practitioner is to recognize the patient's fear as existing and not to deny it, nor to underestimate it. Once this act of conscience has been established, the dentist must then develop a relationship of trust with his patient. Without this relationship of trust, the patient cannot fully adhere to the treatment plan, and therefore the success cannot be optimal. It should be remembered that the patient's fear is counterproductive, its management is therefore essential for the proper conduct of care. It should be remembered that the dentist is in most cases faced with an anguished interlocutor who is rarely aware of the state of his oral health. Listening is essential, the practitioner must undertake psychological support for the patient in a dental office where colors and music are used to create a welcoming, calm space that inspires relaxation and not agitation, benefiting from a pleasant light adapted to each work or waiting space.

\section{ACKNOWLEDGEMENTS AND DISCLOSURES}

The authors declare that they have no potential conflicts of interest to disclose

\section{REFERENCES}

1. Armbruster D, Kirschbaum C, Strobel A: The not-so-bitter pill: Effects of combined oral contraceptives on André C. Psychologie de la peur : craintes, angoisses et phobies. Odile Jacob. 2005

2. Dictionnaire Larousse. 2017

3. Amzlag A. Code de la relation dentiste-patient. Masson. 2007

4. Vaillé H. Cerveau: les mécanismes de la peur. 2005.

5. .Maniglia-Ferreira C., Gurgel-Filho, Bönecker-Valverde G., Moura EH, Deus G., Coutinho-Filho T. Ansiedade odontológica: nível, prevalência e comportamento. RBPS. 2004;17:51-5.

6. Van Wijk A.J., Hoogstraten J. Anxiety and pain during dental injections. J Dent. 2009;37:700-4.

7. American Psychiatric Association (APA). Diagnostic and statistical manual of mental disorders (DMS-IV-TR). Washington, DC: American Psychiatric Association. 2000.

8. Leal C.S., Abreu D., Frenken J.E. Dental anxiety and pain related to art. J Appl Oral Sci.2009; 17:8:4-81678-7757.

9. Binhas E. La gestion globale du cabinet dentaire.2013.

10. Ergonomie du cabinet. Lefidentaire Mag. Dent.2011.

11. Lubos L.C. The Role of Colors in Stress Reduction Liceo Journal of Higher Education Research.2008;5(2):20941064. 
12. Yatishkumar S. et al. Importance of chroma therapy in dentistry, Indian J Dent Adv. 2013; 5(3): $1252-1256$.

13. Umamaheshwari N., Asokan S., Kumaran T.S. Child friendly colors in a pediatric dental practice. J Indian Soc Pedod PrevDent. 2013; 31: 225-8.

14. Goldstein, K. Some experimental observations concerning the influence of colors on the function of the organism. Occup Ther Rehabil.1942; 21: 147-51.

15. Hotwani K., Sharma K. Assessment of the Impact of Colors on Child's Anxiety and Treatment Preference for Local Anesthesia Injections, Journal of Advanced Oral Research. 2017;8(1,2):42-46.

16. Sathyaprasad S., Sruthy P.G., Krishnamoorthy S.H., Vinodhini J., Anjana A .G., Rekha R. et al Behaviour management by colouring exercise using features of cognitive development in children. Internat $\mathbf{J}$ of Current Research. 2018;10(03):67027-67030.

17. Standley J.M. Music research in medical/dental treatment: meta-analysis and clinical applications. J Music Ther.1986;23(2):56-122.

18. .Chandure J., Tamgadge S., Tamgadge A. Effect of music therapy on adult patients undergoing dental treatment procedures. Int Clin Pathol J. 2017;5(4):270-274.

19. Ainscough S.L., Windsor L., Tahmassebi J.F., A review of the effect of music on dental anxiety in children. European Archives of Pediatric Dentistry.2019;20:23-26.

20. Amzalag D.A., Cochet R. Les nouveaux codes de la relation chirurgien-dentiste/patient. 2011:5.

\section{Correspondence:}

Magda Ecaterina Antohe,

$\mathrm{PhD}$, professor, "Grigore T. Popa University of Medicine and Pharmacy Iași, Romania, Faculty of Dental Medicine, Department of Implantology, Removable Dentures, Technology, Discipline of Partial Removable Prostheses, 16 Universității street, magda.antohe@ yahoo.com

Submission: 2 jul 2020

Acceptance: 25 aug 2020 


\title{
Clinical and biological particularities of depressive disorder in women patients
}

\author{
Gavril Radu, Gavril Mihaela Raluca, Dobrin Irina, \\ Traian Carmen, Ștefănescu Cristinel
}

\begin{abstract}
Radu Gavril - MD, Resident in psychiatry, Socola Institute of Psychiatry Iași
Raluca Mihaela Gavril - PhD student, psychiatrist, Socola Institute of Psychiatry Iași Irina Dobrin - MD, PhD, lecturer, psychiatrist, Grigore T. Popa University of Medicine and Pharmacy Iași

Carmen Traian - Specialist Clinical Psychologist, Socola Institute of Psychiatry Iași

Cristinel Ștefanescu - MD, PhD, professor, psychiatrist, Grigore T. Popa University of Medicine and Pharmacy Iași
\end{abstract}

\begin{abstract}
Even though the modern age has offered multiple progress both technological and medical and that includes life expectancy, a series of issues have been noticed within the lifestyle that negatively affects mental health. Although acute depressive disorder is one of the most frequent and invalidating psychiatric disorders from modern times, its origin remains unclear. The prevalence of this disorder has increased in the past 3 decades. Even though this may be caused by errors in diagnostic procedures and later release of patients, it is most probable that there are modern factors that contribute to this increase. Together with the physiopathological mechanisms that involve the role of cerebral monoamines in depression, the inflammatory hypothesis according to which there is a bi-directional relation between depression and inflammation, seems to get more support in the scientific world. Even though there were noted differences both between the clinical chart and the evolution of the depressive disorder in men and women and this is a very important aspect that needs to be considered in the clinical approach of the patient, each being an unique case.
\end{abstract}

\section{KEY WORDS:}

Depressive disorder, cerebral monoamines, inflammatory hypothesis.

\section{INTODUCTION}

A major depressive disorder is one of the most frequent and invalidating psychiatric disorders. Although its etiology remains unclear (1). A meta-analysis that included articles from 1995 to 2016 made clear the connection between different biological and psychological models. Some possible histopathological mechanisms of depression 
ryor include modified neurotransmission, the melancholic

predisposition is anomalies of the hypothalamic-hipofizopredominant. Daily fluctuations in adrenal axes implicated in chronic stress, inflammation, reduced neuroplasticity, and neuronal network dysfunctions. All these proposed mechanisms are tied and interact bidirectionally. Besides, it has been proved that psychiatric stress factors have a direct effect over neuro-development, determining a biological predisposition to depression, while biological factors may lead to psychiatric pathology. Authors suggest that even though there are different phenotypes of depression with different fiziopathological mechanisms, it can be useful that depression is considered to be a single syndrome, in which these mechanisms interact as different nods in a matrix (2).

Numerous studies suggest universal and substantial a women preponderance in depression. There are taken into consideration all the possible hypotheses in this type of phenomenon and the contribution of changing sex roles, social factors, and biological factors are analyzed. While these factors lead to some type of contribution, it has been concluded that it exists a higher biological factor, such as stress receptivity or hyperactivity of the limbic system, that mainly contributes to differentiating of the genre in some expressions of depression and anxiety as well and reflects the impact of gonadal steroids at puberty modification $(3,4)$.

It has been noted that there are differences between the clinical aspect as well as the evolution in recurrent depressive disorder in men and women. Depression in women develops at a younger age, the number of recurrences is higher and the remission periods are longer. Even though, the complete remission is obtained more frequently in men. The most common symptom associated with depression in women is anxiety, while in men disposition, suicidal thoughts are predominant in women, according to a study made bu Ivanets and collaborators (5). Fatigability and the reduction of motivation are most frequent in men. Selfguilt, social isolation, adinamy, permanent fatigue sensation are most frequent in women. Sleep disorders are just as frequent in men and women, but sleep architecture disorders are most frequent in women (6). Somatic symptoms associated with depression are generally more frequent in men, as well as cardiovascular co-morbidities, respiratory, and genital-urinary (7). Men have a greater risk of ethanolic abuse and other psychoactive substances in the attempt of managing the symptoms (8).

Depression symptoms are an epidemic problem that affects different subgroups of women in different environments (9). Even though the depressive symptoms of working women have been rarely studied. In a study made bu Al-Monadall and collaborators, the depressive symptoms and its determinant factors were studied on a 101 working women control group in Jordan, that was recruited in an institution of superior studies. Data about the depressive symptoms of the women were collected, as well as their educational level, the presence of offspring, the sharing of the working place with an intimate partner, the health state, the chronic disease diagnostic, and the husband abuse. The results illustrated that $51.2 \%$ of the women complained of moderate and severe depressive symptoms. The indicated factors classed as important in the prediction of the depressive symptoms were the women's experience with abuse from husbands and chronicle disease diagnostic. A conclusion has been reached according to which the causes of depressive symptoms in women have been classified in the social and 
family environment, instead of the working place. (10).

In a community study made in 2009 in Malaysia, 480 women were evaluated to establish the prevalence and the associated factors with the depressive disorder. Information was collected about the variables in socio-demographic factors, high-risk potential factors (family history and mental health problems, depressive symptoms in life, and associated stress factors), and actual depressive symptoms. Out of the risk factors, the most common risk was the history of depressive manifestations, followed bu actual life stress factors and family history of mental illness. Among the socio-demographic variables used, the health state perceived was of the utmost importance (11). The associated factors with symptoms of depression were found in this study under the discoveries in the Occident, which implies a universality of the phenomenon and the tied factors of the symptoms of depression in women (12). The prevalence of the depression symptoms reported by the study was comparable with the anterior studies $(13,14)$.

The anxiety and depression symptomatology of the adult has to do with numerous factors, that put their stamp over the perspective of life ever since childhood. We are talking here about the family patter, the lifestyle filled with stress and abuse, the family pathological incidence, alcohol consumption, and psychoactive substances (15). Even though, no anterior study has evaluated the mediator role of smoking in association with childhood adversity, anxiety, and depressive symptoms in adult age. Even more, the importance of the variables that confront with the mediator response was rarely demonstrated empirically in psychiatric and social epidemiology. (16).
A representative study, that implicated 4570 people that have as a purpose the evaluation of the role of smoking in adult age in association with the childhood adversity in anxiety and depressive symptoms at adult age and the evaluation of the estimated factors that integrate with the mediator answer (education, alcohol consumption, and social support) (17). Childhood adversity has been associated with a $10 \%$ risk factor in adolescence smoking and 9\% in adult life when education was excluded as a variable confused with the mediator response the indirect effect of childhood adversity over the depressive and anxiety symptoms of adult life have been statistically significative. This study shows the inclusion of potential variables integrated is important in the evaluation of the mediation (18).

Daily basses issues and environment, for instance, urbanization and exposure to noise and chemical pollution and human interface are in a decrease in rapport with the technology that contributes also to the incidence and maintenance of depression. There are necessary studies that exploit the modification of the lifestyle that implicates more elements in the lifestyle (19). While the abusive and incorrect use of medicine and psychological techniques are often blamed, because of the complexity of the disease it is encouraged a more integrative approach of depression and admittance of the fact that the modification of the lifestyle should be a part of the treatment and prevention (20). The children exposed to social adversity, present a higher risk to mental health and physical health in adult life. It is believed that this higher risk is because of the inflammatory process associated with childhood adversity it contributes to many etiological diseases in adults. 
Social adversity in the prenatal period is a risk factor for high inflammation at adult age independent of the adversities of the childhood period. This proof is following the studies that prove the adverse exposure to the maternal environment while gestation has a longer effect on immunity system development (21). If these results reflect a causal association this suggests that the intervention for improving the social conditions and pregnancy environment to promote health to the overall life. It remains necessary for the identification of the mechanisms that link maternal conditions during pregnancy from fetal immunity development and other systems involved in the adaptation of the stress factors (22).

Numerous studies on animals have proven that the systemic or central administration of proinflammatory cytokine induces similar symptomatology with the equivalent of the depressive symptoms in humans. The human population that suffers from inflammatory Cronic diseases like type 2 diabetes, rheumatoid arthritis, cardiovascular diseases have reported increased incidents of depressive disorders. Moreover, the studies measure the terrific expression of cytokines in human patients with depression that have indicated that pro-inflammatory cytokines levels such as alpha tumoral necrose factor, 1 beta interleukin, and 6 interleukins are significantly increased in this clinical population in comparison with the control groups (23). Few studies investigated the inflammatory phenomenon at the brain level in the context of depression and suicidal behavior. Independent groups have reported proof of increased expression of proinflammatory cytokines in cortical regions involved in depression and suicide. How central and peripheric inflammatory phenomenon links to depression and suicide is a good question and needs to be more investigated. (24).

There is a link between immunity function and psychiatric conditions, especially in depressive and anxiety disorders. Psychological stress is a high factor for this kind of disorder and stress. As it was already been demonstrated influences immunity (25). The basolateral amygdala is indispensable for emotion and plays an important role in stress effects on anxiety. Therefore it can be the main mediator of the central nervous system for peripheric immune modification effects on anxiety after stress (26).

The epidemiologic data regarding the prevalence of mood disorders demonstrates that major depressive disorder is approximately twice as frequent in females than in males and usually starts in females who have the reproductive function active (27). To efficiently treat depression in women and to prevent the apparition of the disorder clinicians must understand the specific aspects of feminine gender and mood disorders within the reproduction part of the lifetime in women. Therefore, a biopsychosocial approach should be the primary objective of future practice and research, to result in an integrated etiological model of depression in women. Based on the prevalence of depressive disorder in women, screening, intervention, and diagnostic at the appropriate time should be a priority in public health (28).

\section{MATERIALS AND METHODS}

To evaluate the relationship between inflammation, the intensity of depression somatic and psychiatric comorbidities associated, a retrospective study has been done, comparing statistical analysis, that targets female patients committed in Clinic nr. 5 of the Psychiatric Institute Socola, in 2018, 
(1) with major depressive disorder diagnostic. RESULTS

The demographic data were analyzed, the intensity of depression has been measured through the Hamilton depression scale, VSH values, and suicidal associated risk. From the total number of selected patients, a subgroup of patients has extracted that presented suicidal risk.

The groups of patients who have been selected from the electronic archive of the Socola Institute of Psychiatry and consulting the clinical documents the patients expressed the consent for the usage of personal data in the use of scientific research. The statistical use of data was done in excel and established a correlation between the intensity of depression through the HAMD score and VSH value. Also, it has been used the function of linear regression and statistical analysis ANOVA for highlighting the presence of a significant $\mathrm{p}$, the margin of error being 0.05 .

The valoric, graphic, and percentual analysis followed mainly the VSH values in ascending order, according to the age standards: women group with age between 20-40 years and 4160 years with VSH $>20$ and women $>61$ with $\mathrm{VSH}<30$. Age groups represent the second element in classification. For each patient is being registered the degree of depression (HAMD scale) and anxiety level (HAMA scale), these values being presented for each age group from the moment of being committed, the depression value (HAMD) being considered severe.

Tabel 1. Report male/female committed between 20102018

\begin{tabular}{|l|l|l|l|l|}
\hline Year & $\begin{array}{l}\text { Total of } \\
\text { patients }\end{array}$ & Women & Male & $\begin{array}{l}\text { Women } \\
\text { with } \\
\text { suicidal } \\
\text { risk }\end{array}$ \\
\hline $\mathbf{2 0 1 0}$ & 198 & $93(47 \%)$ & $\begin{array}{l}105 \\
(53 \%)\end{array}$ & 22 \\
\hline $\mathbf{2 0 1 8}$ & 156 & $67(49 \%)$ & $\begin{array}{l}89 \\
(51 \%)\end{array}$ & 15 \\
\hline
\end{tabular}

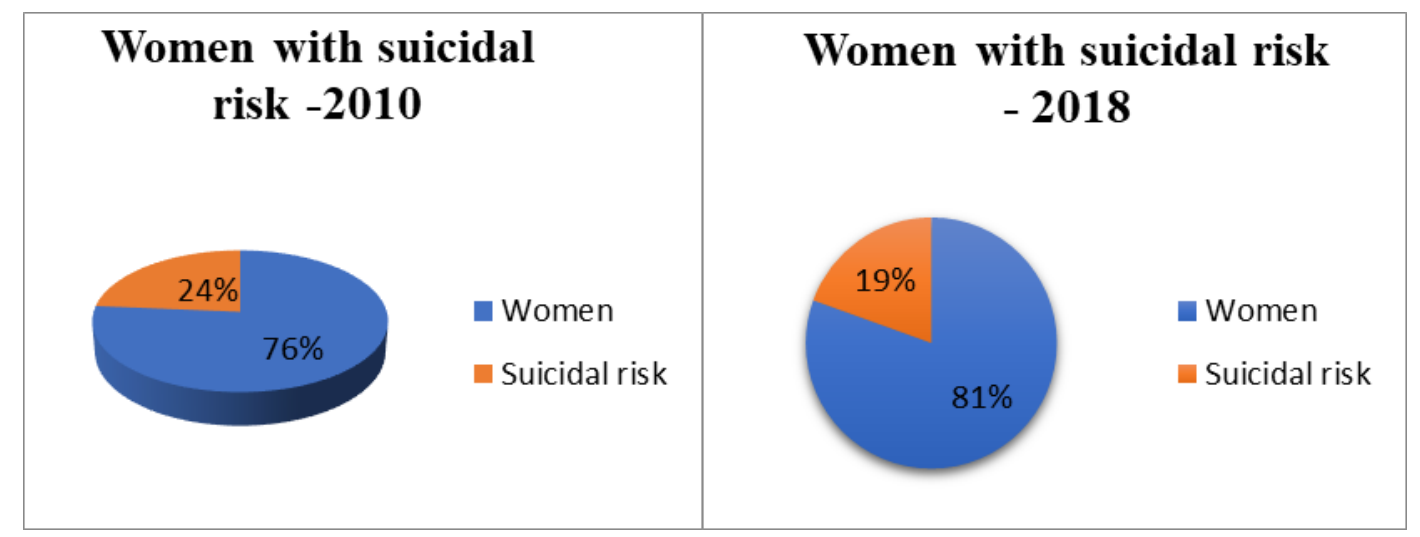

Fig. 1 Report of females committed between 2010-2018

Analyzing the above data we observe the fact that there are no major differences between the number of women and men that have been committed both in 2010 and 2018 the numeric and percentage tendency is higher in men. In $201053 \%$ of the patients are men, over $47 \%$ of women, and in $201851 \%$ are men and $49 \%$ are women.
Regarding patients that have a suicidal risk, it has been noted that there is a diminishing percentage from $24 \%$ out of the total number of women committed in 2010 and $19 \%$ in 2018.

Regarding the environment for the patients committed in 2010 and 2018, the differences are very small, 43 women from the rural 
56/Bulletin of Integrative Psychiatry ONew Series O September 2020 ○ Year XXVI ONo. 3 (86)

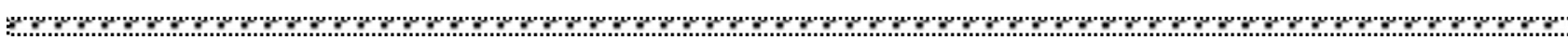
environment and 50 women from the urban rural environment and 35 from urban environment, and in 201832 women from the provenience.

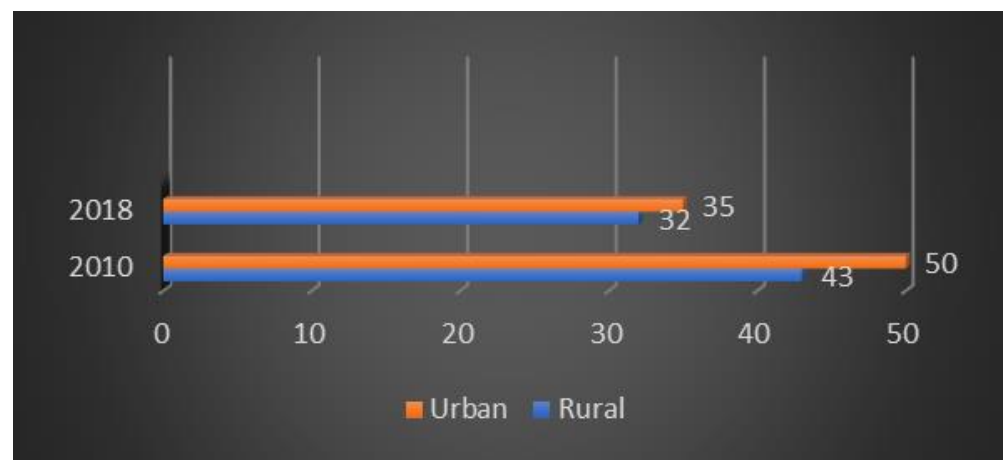

Fig. 2 The origin environment of the patients

The environment doesn't represent an exact variable that influences in a big measure the actual study, considering that the tendency of the population to move from city centers to the outskirts of cities and nearby villages, therefore cannot be classified by the hospital archive, as from rural provenience.

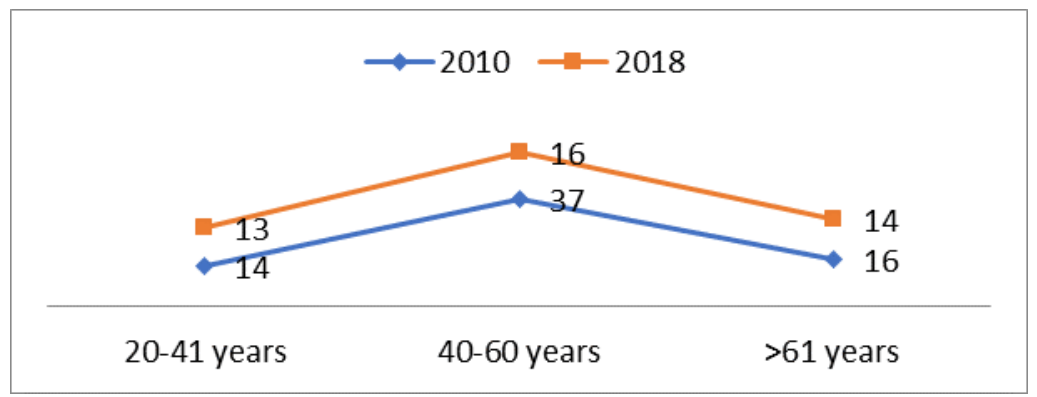

Fig. 3 Distribution by age groups

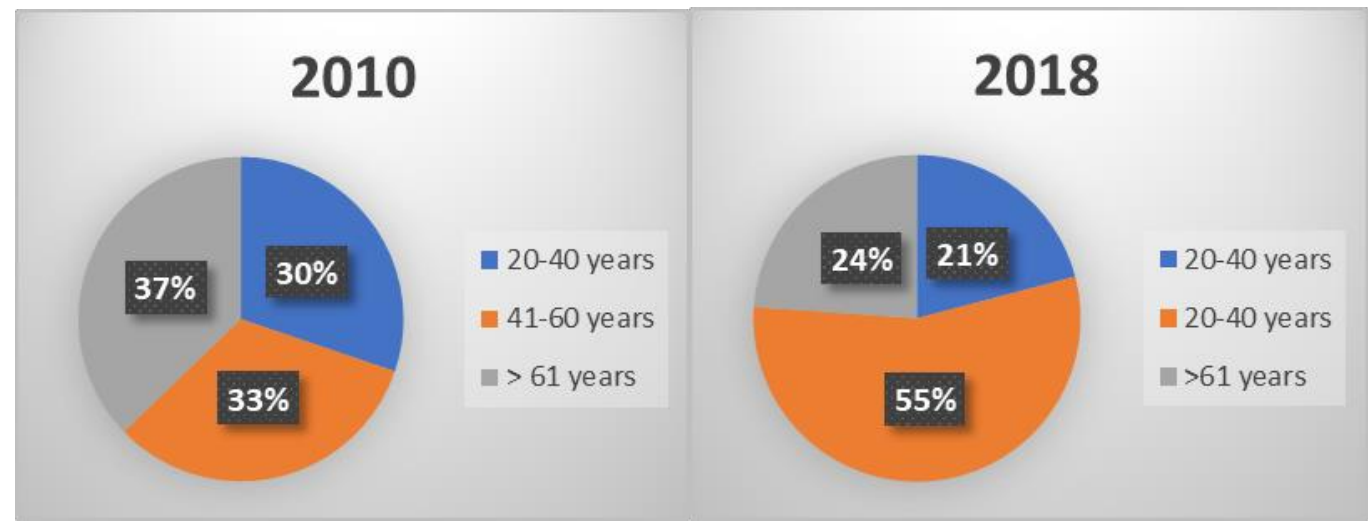

Fig. 4 Repartition of patients according to age groups in 2010 vs 2018

In regarding the distribution depending on age groups, it has been noted that in 2010 as well as in 2018 the fact that the majority of patients are with ages between 41-60 have a significant increase in depression from a groupage 40-60 (from 30\% in 2010 to $55 \%$ in 2018) and a decrease in the number of patients with ages over 61 (from 37\% in 2010 to $24 \%$ in 2018 ). 
Bulletin of Integrative Psychiatry O New Series OSeptember 2020 ○ Year XXVI ONo. 3 (86)/57

s

The application of the Hamilton scale for patients with depression in the study revealed scores with less than 27 points and patients that do not have 25 scores were not included in the study. The average of the scores in depression in 2018 for women registered a HAMD value of $32.04 \pm 2.23$, in HAMA case $26.3 \pm 1.33$ with a VSH value of $19.17 \pm 8.17$. Following the same parameters in 2010, the average score for depression calculated with the HAMD scale in women in 2010 with the average value for HAMD was $31,14 \pm 2,56$, for HAMA - 25,51 $\pm 1,46$ and an average value for VSH of $22,25 \pm 6,76$.

To identify and study the relationship between VSH and HAMD from the women study group without suicidal thoughts in 2010 was calculated the PEARSON coefficient $\mathrm{r}=0,6 \mathrm{~m}$ value that suggests a moderate correlation between the 2 variables. The positive sign suggests that the low score from the first variable correlates with the low score of the second variable. The average score from the first variable correlates with the average score from the second variable. The higher score from the first variable correlates with the higher score from the second variable. To find out the $\mathrm{p}$-value it has been applied the linear regression function in EXCEL. The statistical analysis ANOVA observers the HAMD values and VSH $\mathrm{F}(1,20)=0,5$, at a $\mathrm{p} 0,5$.

\begin{tabular}{|l|l|l|}
\hline $\begin{array}{l}\text { Regression } \\
\text { Statistics }\end{array}$ & Column 1 & \\
\hline Multiple R & 0.783553718 & \\
\hline R Square & 0.613956429 & \\
\hline $\begin{array}{l}\text { Adjusted R } \\
\text { Square }\end{array}$ & 0.59465425 & \\
\hline $\begin{array}{l}\text { Standard } \\
\text { Error }\end{array}$ & 2.121950008 & \\
\hline Observations & 22 & \\
\hline
\end{tabular}

\begin{tabular}{|l|l|l|l|l|l|}
\hline ANOVA & & & & & \\
\hline & $d f$ & $S S$ & $M S$ & $F$ & Significance $F$ \\
\hline Regression & 1 & 143.2192905 & 143.2193 & 31.807 & $1.60915 \mathrm{E}-05$ \\
\hline Residual & 20 & 90.05343676 & 4.502672 & & \\
\hline Total & 21 & 233.2727273 & & & \\
\hline
\end{tabular}

\begin{tabular}{|l|l|l|l|l|l|l|}
\hline Column 1 & Coefficients & Standard Error & t Stat & P-value & Lower 95\% & Upper 95.0\% \\
\hline Intercept & 21.8448610 & 1.887858266 & 11.5712 & $2.58 \mathrm{E}-10$ & 17.906857 & 25.78286436 \\
\hline VSH & 0.41198017 & 0.0730484 & 5.63982 & $1.61 \mathrm{E}-05$ & 0.2596038 & 0.564356469 \\
\hline
\end{tabular}

Similarly we evaluated the group of patients without suicidal thoughts in 2018. The PEARSON coefficient $r-0,52$ suggesting a strong correlation between VSH and HAMD. Statistical analysis ANOVA indicates $\mathrm{F}(1,47)=0,9$ at a $\mathrm{p}=0,9$.

\begin{tabular}{|l|l|}
\hline Regression Statistics & \\
\hline Multiple R & 0.726697 \\
\hline R Square & 0.528089 \\
\hline Adjusted R Square & 0.518048 \\
\hline Standard Error & 1.467662 \\
\hline Observations & 49 \\
\hline
\end{tabular}

\begin{tabular}{|l|l|l|l|l|l|}
\hline ANOVA & & & & & \\
\hline & $D f$ & $S S$ & $M S$ & $F$ & Significance $F$ \\
\hline Regression & 1 & 113.2912 & 113.291 & 52.5949 & $3.39 \mathrm{E}-09$ \\
\hline Residual & 47 & 101.2395 & 2.154031 & & \\
\hline Total & 48 & 214.5306 & & & \\
\hline
\end{tabular}


58/Bulletin of Integrative Psychiatry ONew Series O September 2020 ○ Year XXVI ONo. 3 (86)

\begin{tabular}{|l|l|l|l|l|l|l|l|}
\hline & Coefficients & $\begin{array}{l}\text { Standard } \\
\text { Error }\end{array}$ & $t$ Stat & P-value & $\begin{array}{l}\text { Lower } \\
95 \%\end{array}$ & $\begin{array}{l}\text { Lower } \\
95.0 \%\end{array}$ & Upper 95.0\% \\
\hline Intercept & 28.2468 & 0.529814 & 53.314 & $1.08 \mathrm{E}-43$ & 27.18 & 27.181 & 29.31269 \\
\hline VSH & 0.20128 & 0.027755 & 7.2522 & $3.39 \mathrm{E}-09$ & 0.145 & 0.1454 & 0.257122 \\
\hline
\end{tabular}

As we observe in the above graphic the highest values of $\mathrm{VSH}$ are associated with higher values in HAMD, sustaining the hypothesis that inflammation and depression are in a bidirectional relationship.
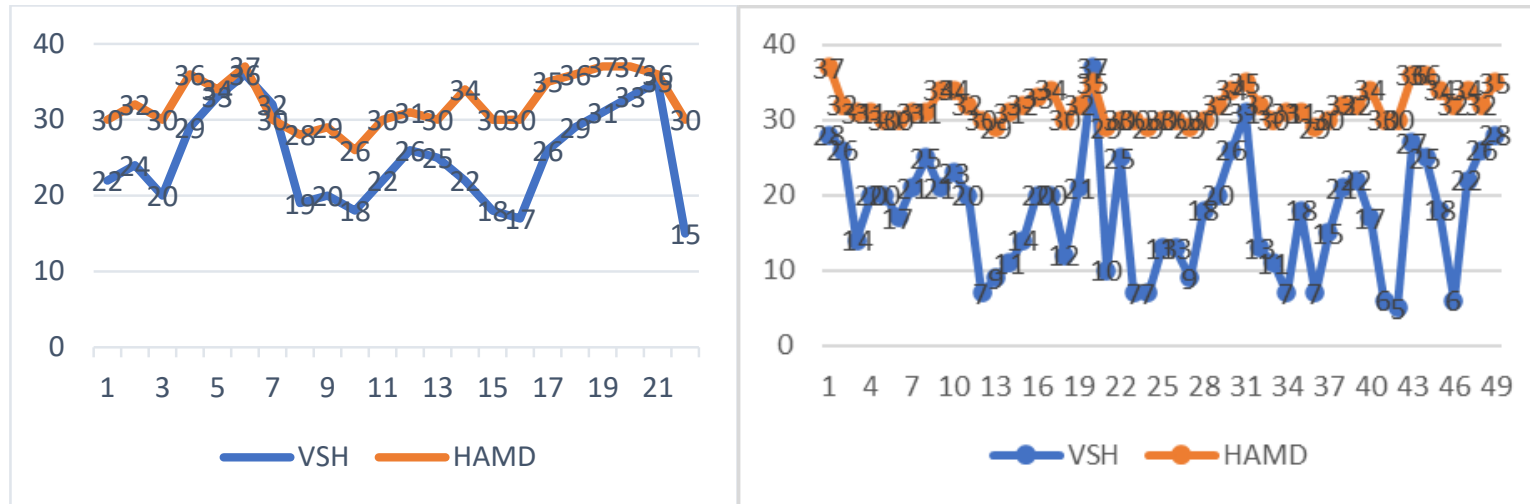

Fig. 5 Correlations VSH-HAMD in patients

Without suicidal thoughts in 2010-2018

\begin{tabular}{|l|l|}
\hline Regression Statistics & Column 1 \\
\hline Multiple R & 0.2071492 \\
\hline R Square & 0.0429108 \\
\hline Adjusted R Square & 0.0368467 \\
\hline Standard Error & 2.4942093 \\
\hline Observations & 14 \\
\hline
\end{tabular}

We analyzed the women group with suicidal risk in 2017. For the 14 patients we identified an average value of HAMD score $33 \pm$ for standard deviation 2.44 an average value of VSH of 24,9 \pm deviation standard 7,57 and for HAMA an average value $26,37 \pm$ deviation standard 1,3 .

\begin{tabular}{|l|l|l|l|}
\hline & VSH & HAMD & HAMA \\
\hline Media & 24.928571 & 33 & 26.375 \\
\hline Dev. St. & 7.5799765 & 2.44 & 1.30247 \\
\hline
\end{tabular}

The Pearson coefficient $r=0,04$, a value that suggests a moderate correlation between the two variables. To find out the $\mathrm{p}$-value it has been applied to the linear regression function in Excel, the significant threshold chosen was $<0,05$. We can observe the ANOVA statistical analysis between the HAMD and VSH $\mathrm{F}(1,12)=0,5$ values at $\mathrm{p} 0,1$.

\begin{tabular}{|l|l|l|l|l|l|}
\hline & & & & & \\
\hline Column1 & Coefficients & Standard Error & t Stat & P-value & \\
\hline Intercept & 31.331261 & 2.3706983 & 13.21605 & $1.64 \mathrm{E}-08$ & \\
\hline VSH & 0.0669408 & 0.0912627 & 0.733496 & 0.477342 & \\
\hline ANOVA & & & & & \\
\hline & $D f$ & $S S$ & $M S$ & $F$ & Significance F \\
\hline Regression & 1 & 3.34704 & 3.34704 & 0.538016 & 0.477342 \\
\hline Residual & 12 & 74.65296 & 6.22108 & & \\
\hline Total & 13 & 78 & & & \\
\hline
\end{tabular}


Bulletin of Integrative Psychiatry O New Series OSeptember 2020 ○ Year XXVI ONo. 3 (86)/59 s s Similarly, we analyzed the women group with The statistical analysis continues to suicidal risk from the year 2010 and identified a HAMD average value of $32.181 \pm 3,33$ standard deviation with an average VSH value of $25,090 \pm 6,33$ standard deviation with an average HAMA value of $25,545 \pm$ 1,47 standard deviation.

Also, the Person coefficient has been calculated with an $\mathrm{r}=0,61$, witch suggests a evidentiate a $\mathrm{p}=0,5$ significant statistic.

strong correlation between HAMD value and VSH value.

\begin{tabular}{|l|l|l|l|l|l|}
\hline & $D f$ & $S S$ & $M S$ & $F$ & Significance F \\
\hline Regression & 1 & 143.2192905 & 143.219 & 31.8076 & $1.60915 \mathrm{E}-05$ \\
\hline Residual & 20 & 90.05343676 & 4.50267 & & \\
\hline Total & 21 & 233.2727273 & & & \\
\hline Column1 & Coefficients & Standard Error & $\boldsymbol{t}$ Stat & $\boldsymbol{P}$-value \\
\hline Intercept & 21.8448610 & 1.887858266 & 11.5712 & $2.58 \mathrm{E}-10$ \\
\hline VSH & 0.41198017 & 0.0730484 & 5.63982 & $1.61 \mathrm{E}-05$ \\
\hline
\end{tabular}

As it is indicated in the graphical representation from above in female cases with suicidal risk selected in 2010 and 2018,

\begin{tabular}{|l|l|}
\hline Regression Statistics & Column1 \\
\hline Multiple R & 0.78355371 \\
\hline R Square & 0.61395642 \\
\hline Adjusted R Square & 0.59465425 \\
\hline Standard Error & 2.12195000 \\
\hline Observations & 22 \\
\hline
\end{tabular}

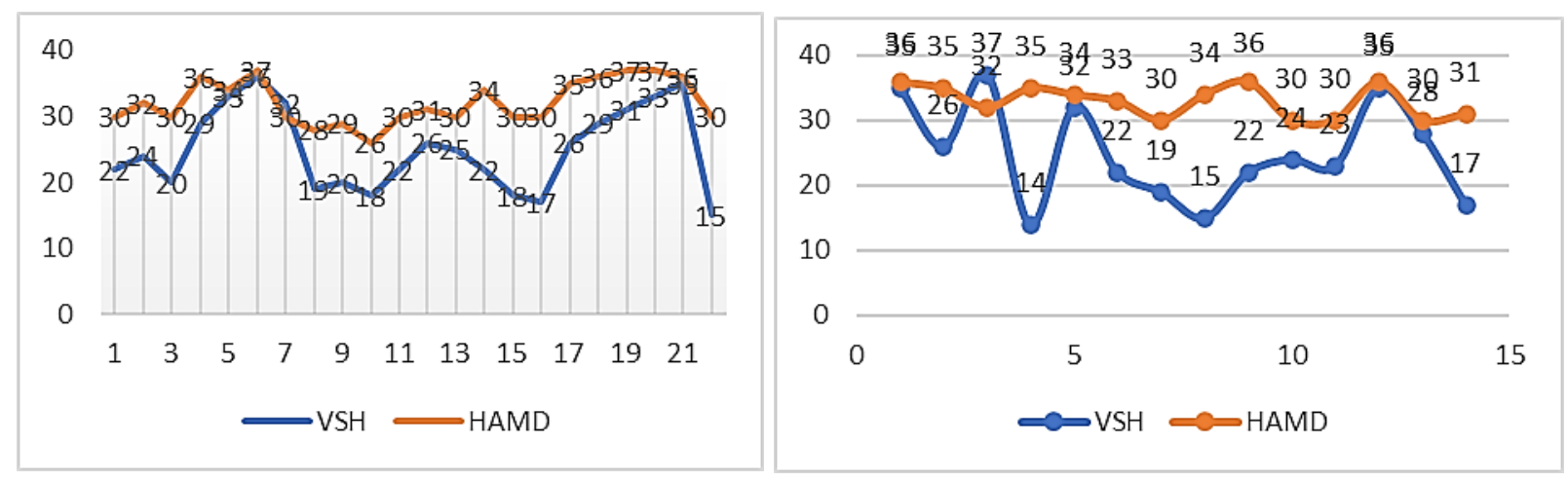

Fig. 6 Correlations VSH-HAMD in patients with suicidal thoughts in 2010-2018

\section{CONCLUSIONS}

Although the etiology of depression and the inflammatory hypothesis are subjects that need to be investigated furthermore we can conclude that between inflammation and depression there is an important link.

We noticed that in patients with suicidal thoughts the correlation between increased values of HAMD score and increased VSH value is stronger than in patients with depression without suicidal thoughts.

Analyzing the demographical data we did not see particularities between women with depression. In 2010 as well as in 2018 the distribution was the same using the parameter origin environment. 


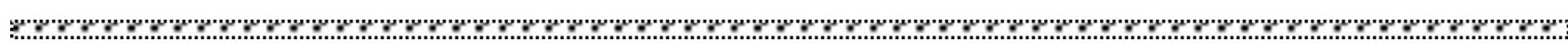
If in 2010 the patient's repartition depending on groupage did not note a significant difference in 2018 we see an important increase in the number of patients age between 40-60 and a diminishing in the number of patients with age over 60 .

\section{ACKNOWLEDGEMENTS AND DISCLOSURES}

The authors have no potential conflict of interests to disclose.

\section{REFERENCES}

1. Peretti S, Judge R, Hindmarch I. Safety and tolerability considerations: tricyclic antidepressants vs. selective serotonin reuptake inhibitors. Acta Psychiatr Scand Suppl. 2000.

2. Jason D, Keshaven M, The neurobiology of depression: An integrated view, Asian Journal of Psychiatry. 2017;27:101-111.

3. Parker G, Brotchie H, Gender differences in depression, Int Rev Psychiatry.2010;22(5):429-36.

4. Ioannidis JP. Effectiveness of antidepressants: an evidence myth constructed from a thousand randomized trials? Philos Ethics Humanit Med. 2008.

5. Ivanets $\mathrm{N}$, Tyuvina $\mathrm{N}$, Voronina $\mathrm{O}$, Balbanova $\mathrm{V}$, Comparative evaluation of depressive disorders in women and men, Zh Nevrol Psikhiatr Im S S Korsakov. 2018;118(11):15-19

6. Park L, Zarate C, Depression in primary care setting, N Engl J Med. 2019;380(6):559-568.

7. Gavin NI, Gaynes BN, Lohr KN, Meltzer-Brody S, Gartlehner G, Swinson T. Perinatal depression: a systematic review of prevalence and incidence. Obstet Gynecol. 2005;106:1071-83.

8. Talge NM, Neal C, Glover V. The early stress, translational research, and prevention science network: fetal and neonatal experience on child and adolescent mental health. Antenatal maternal stress and long-term effects on child neurodevelopment: how and why? J Child Psychol Psychiatry. 2007;48:245-61.

9. Elghossain T, Bott S, Akik C, Obermeyer CM., Prevalence of intimate partner violence against women in the Arab world: a systematic review., BMC Int Health Hum Rights. 2019;19(1):29. doi: 10.1186/s12914-019-0215-5

10. H Al-Modallal, J Abuidhail, A Sowan, A Al-Rawashdeh, Determinants of depressive symptoms in Jordanian working women, J Psychiatr Ment Health Nurs. 2010;17(7):569-76.

11. Meriam Omar Din ${ }^{1}$, Noraini M Noor, Prevalence, and factors associated with depressive symptoms in Malay women, Women Health. 2009;49(8):573-91.

12. Cheah YK, Azahadi M, Phang SN, Abd Manaf NH. Sociodemographic, Lifestyle, and Health Factors Associated With Depression and Generalized Anxiety Disorder Among Malaysian Adults. J Prim Care Community Health. 2020;11:2150132720921738. doi:10.1177/2150132720921738.

13. Sheikh MA. Confounding and Statistical Significance of Indirect Effects: Childhood Adversity, Education, Smoking, and Anxious and Depressive Symptomatology. Front Psychol. 2017;8:1317.

14. Slopen N, Loucks EB, Appleton AA, et al. Early origins of inflammation: An examination of prenatal and childhood social adversity in a prospective cohort study. Psychoneuroendocrinology.2015;51:403-413. doi:10.1016/j.psyneuen.2014.10.016

15. Agerup T., Lydersen S., Wallander J., Sund A. M. Maternal and paternal psychosocial risk factors for clinical depression in a Norwegian community sample of adolescents. Nord. J. Psychiatry 2015;69:35-41. 10.3109/08039488.2014.919021.

16. Albers A. B., Biener L.The role of smoking and rebelliousness in the development of depressive symptoms among a cohort of Massachusetts adolescents. Prev. Med.2002;34, 625-631.

17. Sheikh MA. Confounding and Statistical Significance of Indirect Effects: Childhood Adversity, Education, Smoking, and Anxious and Depressive Symptomatology. Front Psychol. 2017;8:1317.

18. Baldassari A. R., Cleveland R. J., Callahan L. F. Independent influences of current and childhood socioeconomic status on health outcomes in a North Carolina family practice sample of arthritis patients. Arthritis Care Res. (Hoboken). 2013;65: 1334-1342.

19. Jimenez MP, Wellenius GA, Subramanian SV, et al. Longitudinal associations of neighborhood socioeconomic status with cardiovascular risk factors: A 46-year follow-up study. Soc Sci Med. 2019;241:112574. doi:10.1016/j.socscimed.2019.112574 
20. Steiner J, Bielau H, Brisch R et al. Immunological aspects in the neurobiology of suicide: elevated microglial density in schizophrenia and depression is associated with suicide. J Psychiatr Res. 2008;42:151-157.

21. Pandey GN, Rizavi HS, Ren X, Fareed J, Hoppensteadt DA, Roberts RC, et al. Proinflammatory cytokines in the prefrontal cortex of teenage suicide victims. J Psychiatr Res.2012;46:57-63. doi:10.1016/j.jpsychires.2011.08.006

22. Howren MB, Lamkin DM, Suls J. Associations of depression with C-reactive protein, IL-1, and IL-6: a metaanalysis. Psychosom Med.2009; 71:171-86.

23. Slopen N, Loucks EB, Appleton AA, et al. Early origins of inflammation: An examination of prenatal and childhood social adversity in a prospective cohort study. Psychoneuroendocrinology.2015;51:403-413.

24. Rau AR, Chappell AM, Butler TR, Ariwodola OJ, Weiner JL. Increased Basolateral Amygdala Pyramidal Cell Excitability May Contribute to the Anxiogenic Phenotype Induced by Chronic Early-Life Stress. J Neurosci. 2015;35(26):9730-9740.

25. Jimenez MP, Wellenius GA, Subramanian SV, et al. Longitudinal associations of neighborhood socioeconomic status with cardiovascular risk factors: A 46-year follow-up study. Soc Sci Med. 2019;241:112574.

26. De Guingand DL, Palmer KR, Snow RJ, Davies-Tuck ML, Ellery SJ. Risk of Adverse Outcomes in Females Taking Oral Creatine Monohydrate: A Systematic Review and Meta-Analysis. Nutrients. 2020;12(6):1780.

27. Van der Waerden JE, Hoefnagels C, Hosman CM. Psychosocial preventive interventions to reduce depressive symptoms in low-SES women at risk: a meta-analysis. J Affect Disord. 2011;128(1-2):10-23.

28. Hagerty BM. Advances in understanding major depressive disorder. J Psychosoc Nurs Ment Health Serv. 1995;33(11):27-34.

Correspondence:

Raluca Mihaela Gavril,

PhD student, psychiatrist, Socola Institute of Psychiatry Iasi, gavrilradu25@gmail.com

Submission: 2 aug 2020

Acceptance: 4 sep 2020 



\title{
Correlations between anxiety levels, gastrointestinal manifestations and social isolation following the lockdown due to coronavirus outbreak in Romanian students
}

\author{
Alexandrina Curpan, Alexandra Săvucă, Luminița \\ Hriscu, Alin Ciobîcă, Daniel Timofte, Peiu Sorin Nicolae
}

Alexandrina Curpan - Department of Research, Faculty of Biology, "Alexandru Ioan Cuza" University of Iași

Alexandra Săvucă - Department of Research, Faculty of Biology, "Alexandru Ioan Cuza" University of Iași

Luminița Hriscu - Faculty of Veterinary Medicine, University of Agricultural Sciences and Veterinary Medicine "Ion Ionescu de la Brad" of Iași

Alin Ciobîcă - PhD, researcher, Department of Research, Faculty of Biology, "Alexandru Ioan Cuza" University of Iași

Daniel Timofte - PhD, professor, Faculty of Medicine, "Grigore T. Popa" University of Medicine and Pharmacy

\begin{abstract}
Background: People choosing to follow a university degree are exposed to different degrees of stress and anxiety that might be higher of lower depending on the level of study, field of interest and even age and sex. It is a well-known fact that social isolation with little to no contact with other people has an impact on the mental health of any individual.Objective: The aim of this study was to evaluate the level of anxiety and its gastrointestinal impact as a consequence of the lockdown caused by Covid-19.Method: A cross-sectional study was conducted by using a single questionnaire representing a variety of the Hamilton scale for anxiety (HAM-A).Results: Out of a total of 38 results, 17 results were excluded as they were composed of people that are not students, but belong to the working and retired class.Conclusions: There seems to be a correlation between anxiety levels, gastrointestinal manifestations and social isolation following the lockdown due to coronavirus outbreak, as observed in this preliminary study of ours in Romanian students, considering that more than half of the participants $(61,91 \%)$ have reported mild to severe symptoms in all 14 categories of the scale and we observed that more than $50 \%$ of participants have reported mild to moderate severity in all of the items within the gastrointestinal category.
\end{abstract}


Anxiety, IBS, lockdown, covid-19, social isolation, Hamilton scale for anxiety.

\section{INTRODUCTION}

Modern society brings a high degree of pressure on the shoulders of modern individuals whether it be work or moneyrelated, school-related or simply day-to-day survival. These problems are diminished or amplified based on the social and cultural expectations, home country, financial status, family, age and even sex. Therefore, these types of worries might lead to different degrees of anxiety some being beneficial (it can give a warning of a possible dangerous situation) and some not so much with the risk of developing general/social anxiety disorders, major anxiety disorders or even suicidal thoughts if anxiety is combined with burnout for example (1). People in general tend to avoid situations that might trigger an uncomfortable degree of anxiety or that can worsen it (like the aforementioned factors) (2) Therefore anxiety disorders are a common trait of modern society at a global level (3). Anxiety becomes a disorder when symptoms like physical weakness, poor memory, fear (especially at the level of phobias), palpitations, gastrointestinal manifestations, poor concentration etc. become chronic making the individual feel uncomfortable and helpless (4).

Recent studies have suggested that there is a connection between gastrointestinal conditions and psychological symptoms, with patients with irritable bowel syndrome for example, a disorder characterized by abdominal pain and alterations in bowel habits, presenting elevated levels of anxiety, depression and somatization compared to healthy individuals(5). When it comes to gastrointestinal symptoms it is possible that by using samples from a clinical setting to not faithfully reproduce a general representative of the population with gastrointestinal symptoms due to the possibility of the selected patients to be more prone to these type of symptoms and to psychological ones as well, therefore the use of random selected participants might lead to different outcomes when anxiety and depression scales are applied. A study performed in Norway on 62651 individuals has revealed anxiety and depression as risk factors for 4 gastrointestinal symptoms (diarrhea, nausea, heartburn and constipation) while observing that anxiety is increasing the risk more than depression (6).

A pandemic is an epidemic that spreads globally, its spread being influenced by various factors with the ease of transmission from one person to the next and the movement of people being the most important ones (7). Sars-cov-2, the novel virus responsible for covid-19, was thought to be a simple flu virus in a specific region of China and has rapidly spread globally thanks to the wonders of modern transportation reaching the level of a pandemic within weeks from his first public appearance. So far there are no efficient or standard treatments for this virus, but several antivirals and vaccines are in different stages of clinical trials. A pandemic of this proportion has determined all countries to enter in complete lockdown with borders, school and any leisure facilities closed, meaning the possibilities of freely moving, socializing or simply going for a walk becoming highly restricted (8).

After performing a literature search, we observed that it is suggested that restrictive measures as the before mentioned ones have an impact on the psychological status of people as well as their emotional response to 
the crisis at hand $(9,10)$. Similar studies conducted during previous outbreaks like MERS (2012) (11), H1N1 (in 2009) (12) and SARS (2002-2003) (13) on general populations have highlighted high levels of anxiety, avoidance behaviors and worries about the infection. This only supports the idea that during an outbreak, in addition to preventing the spread, an extensive attention should also be placed upon the public health response with focus on psychological problems (14). A study conducted on university students during the SARS epidemic in Hong Kong to asses anxiety levels has described higher anxiety levels in students from the teaching hospital where the outbreak occurred and lower in students of other university situated $20 \mathrm{~km}$ away (15).

A cross sectional study performed on local residents in Liaoning Province, China during the covid-19 pandemic, targeting mental health observed that more than half of the participants did not feel helpless due to the pandemic, but at the same time $52,1 \%$ felt horrified and apprehensive. They also observed an increased care amongst family members with only a mild stressful impact (16). A study performed on undergraduate and postgraduate students from University of Jordan to see if there is a correlation between conspiracies theories and anxiety levels regarding covid-19 pandemic has revealed a correlation between lower level of knowledge and higher levels of anxiety with females and participants with low income being more prone to believe that the disease is a conspiracy thus higher anxiety (17).

Therefore, we planned this study to investigate the levels of anxiety and any possible gastrointestinal manifestation among individuals from different age groups at the beginning of the lockdown and to identify the most common symptoms.

\section{MATERIAL AND METHODS}

A cross-sectional study was performed in the north-eastern region of Romania on students enrolled at University of Agricultural Sciences and Veterinary Medicine „Ion Ionescu de la Brad" of Iasi that expressed a desire to be part of the study one week (22 of march 2020) after the instauration of the lockdown in Romania. This study was approved by the Ethical committee of USAMV Iasi. The questionnaire was performed online using Google Forms by sending the targeted individuals an accession link to it with the first question being either they want to be part of the study or not. Participants were enrolled in different cycle of studies starting from bachelor and going till $\mathrm{PhD}$ level as well as in different faculties or departments. Medical history was not an exclusion criteria as we wanted to measure the anxiety levels on a general setting rather than having a well-controlled study group. Before handing over the accession link, all participants were informed about the aim and objective of the study. We didn't have any dropouts and we managed to get a diverse group of participants in age and sex.

The survey included social and demographic information of the participants (age, sex, locality and social class student/working/retired) and by applying Hamilton scale for anxiety (HAM-A) we gathered information like fears, insomnia, depression, cardiovascular, gastrointestinal, respiratory symptoms etc. The test had no time limit, but the time required to fill it in is about 20 minutes.

\section{RESULTS}

A total of 38 individuals participated in the study, with ages between 20 and 60, but we excluded 17 of them based on whether they were studying at the moment or not, leaving us with 21 final participants - 16 females and 

age group.

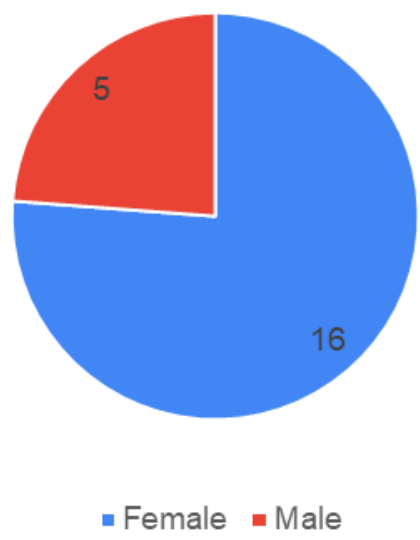

Fig. 1 Sex distribution of participants. Out of 21 final participants, 16 are females with 15 between 20-30 years old and 1 in the 30-40 age group and 5 males all in the 20-30 age group.

After gathering all of the results and calculating the score according to the HAM-A scale, the results were divided into mild severity (a score <17), mild to moderate severity (score of 18-24) and moderate to severe (25-30). Most participants fell between the limits of mild to moderate category $(42,86 \%)$, followed by mild severity $(38,10 \%)$ and ultimately moderate to severe $(19,05 \%)$ (Figure 2).

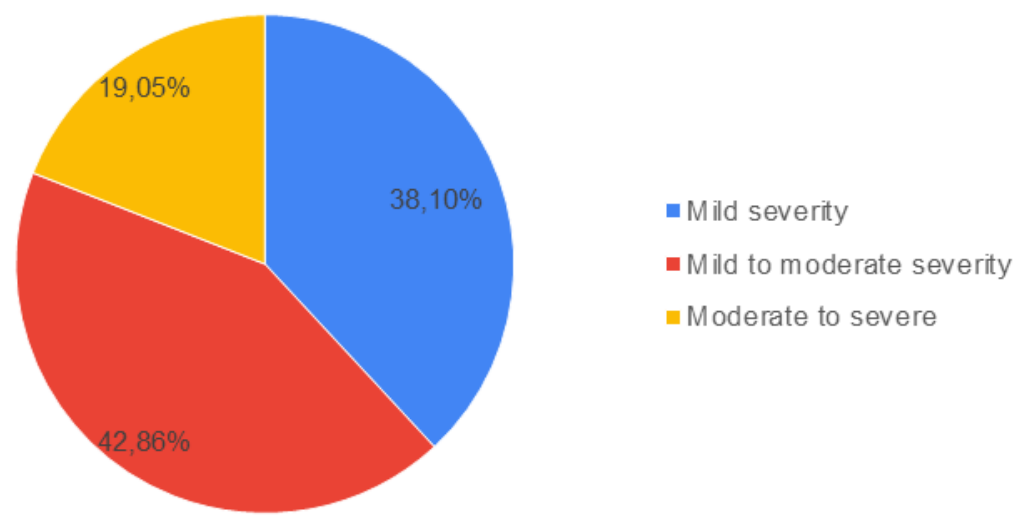

Fig. 2 Score distribution. $38,10 \%$ of score results are corresponding to mild severity, $42,86 \%$ to mild to moderate severity and $19,05 \%$ to moderate to severe.

The average value is 18 , with the lowest score standing at 7 and the highest at 33, with the rest of the values being in normal limits compared to the standard deviation of the mean (Figure 3). 


\section{HAM-A results}

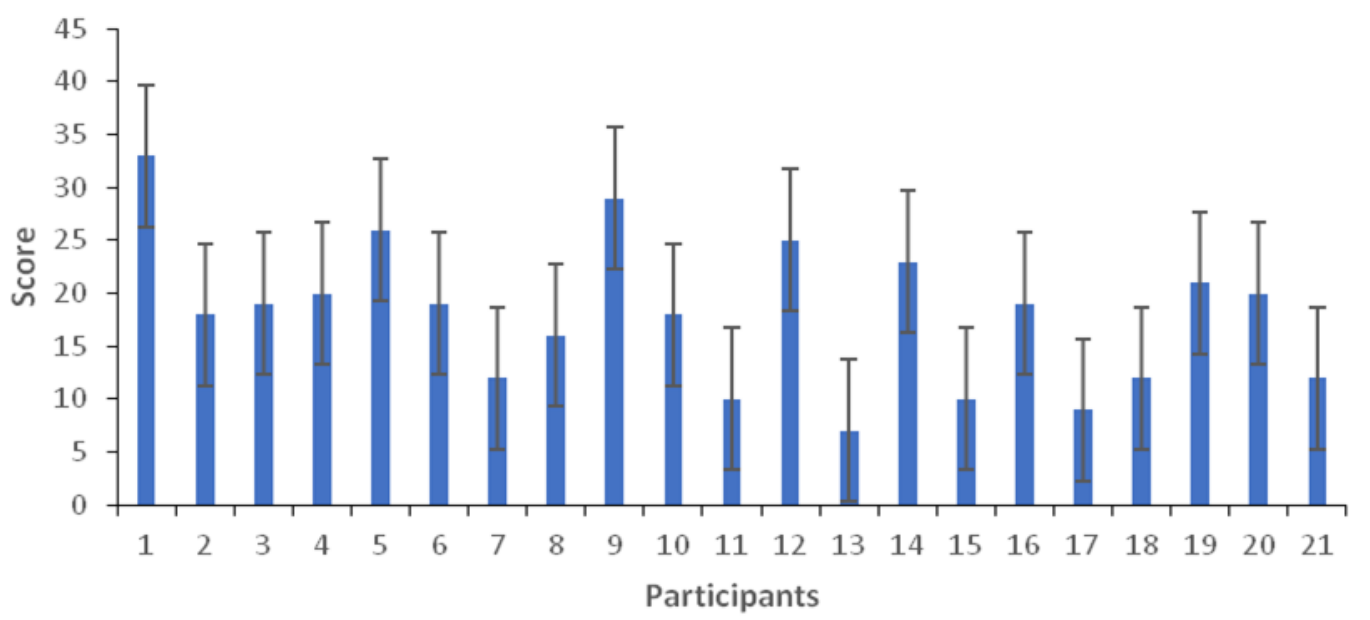

Fig. 3 HAM-A results. The results of a number of 21 participants with a mean score of 18 and a standard deviation of 6,711752 the lowest score (7) belonging to participant number 13 and the highest (33) to participant number 1 . All the other vales fall between normal limits.

When it comes to gastrointestinal manifestations of anxiety(difficulty in swallowing, wind abdominal pain, burning sensations, abdominal fullness, nausea, vomiting, borborygmi, looseness of bowels, loss of weight, constipation) based on the scores given by participants on the symptoms taken all together, the values varied from absent to moderate (Figure 4).

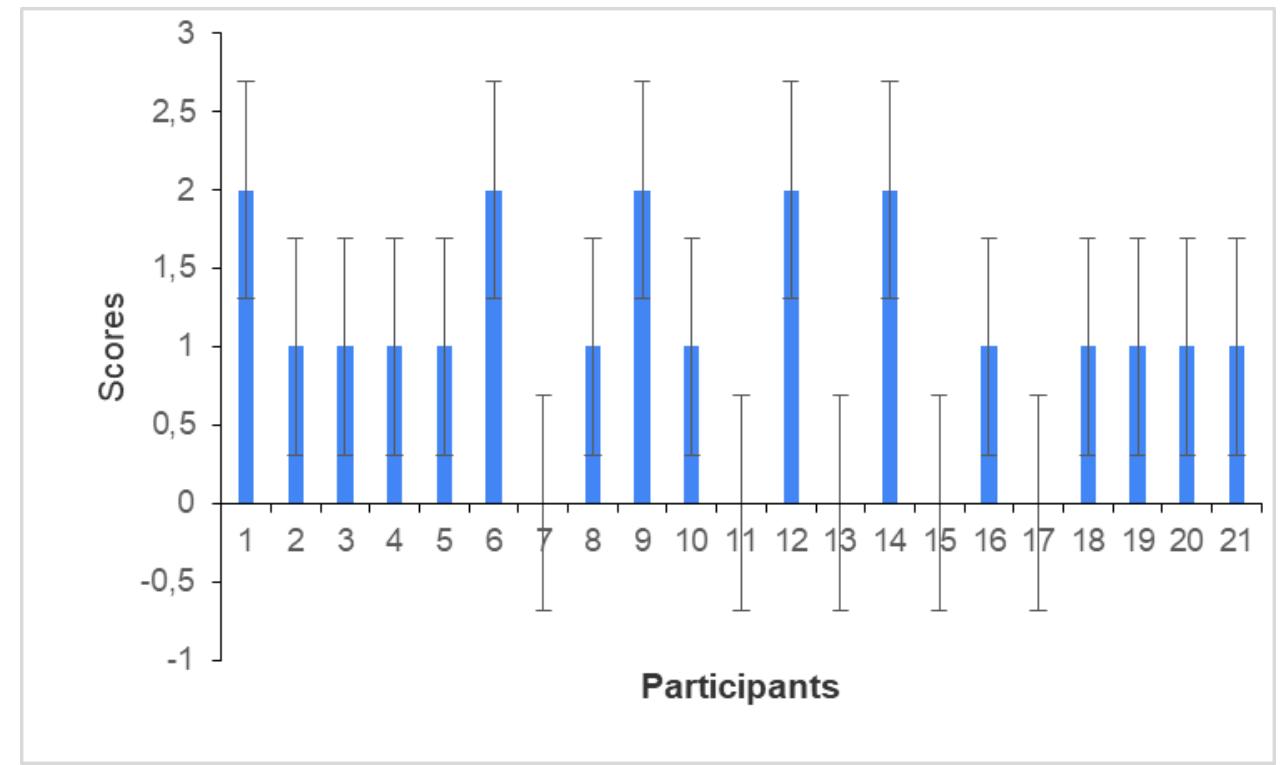

Fig.4 Gastrointestinal symptoms. Score given by participants regarding gastrointestinal symptoms, lowest score being 0 (absent symptoms) and highest 2 (moderate symptoms).

The mean value is 1 and the standard deviation is 0.69 , all values fall between normal limits. When it comes to symptoms taken separately the most common and highest ranked ones were borborygmi (with a mean score of 1.47), abdominal pain (mean score of 1.428) and burning sensations and 
1.23). Overall, the severe symptoms have the symptoms were absent to moderate in the been registered only 4 times in 3 participants majority of participants.

and were for borborygmi, abdominal pain,

\section{CONCLUSIONS}

There seems to be a correlation between anxiety levels, gastrointestinal manifestations and social isolation following the lockdown due to coronavirus outbreak, as observed in this preliminary study of ours in Romanian students, considering that more than half of the participants $(61,91 \%)$ have reported mild to severe symptoms in all 14 categories of the scale, with almost half $(42,86 \%)$ reporting at least moderate symptoms in severity.

When the focus was put onto gastrointestinal manifestations, we observed that more than $50 \%$ of participants have reported mild to moderate severity in all of the items that fall within this category. As mentioned at the beginning of this article, 4 specific symptoms have been previously correlated to be increased by anxiety, those symptoms being diarrhea, nausea, heartburn and constipation. We also observed in our preliminary study, a general prevalence of two of them - heartburn and nausea, but the most common one and with the highest mean value was borborygmi which might be associated with certain bowel disorders, like irritable bowel syndrome when is present with other signs as well as it is not enough on its own. Our study is limited by the number of subjects and the fact that we did not analyze their general anxiety level before the lockdown. Therefore, we suggest that more studies should be made on this topic and maybe on a larger scale that might give a clearer correlation between anxiety levels, gastrointestinal manifestations and social isolation.

\section{ACKNOWLEDGMENTS}

A.C. was supported by a research grant for Young Teams offered by UEFISCDI Romania, no. PNIII-P1-1.1-TE-2016-1210, contract no. 58 from 02/05/2018, called "Complex study regarding the interactions between oxidative stress, inflammation and neurological manifestations in the pathophysiology of irritable bowel syndrome (animal models and human patients).

\section{REFERENCES}

1. Koutsimani P, Montgomery A, Georganta K. The relationship between burnout, depression, and anxiety: A systematic review and meta-analysis. Vol. 10, Frontiers in Psychology. Frontiers Media S.A.; 2019

2. Parekh R. What Are Anxiety Disorders?. 2017. https:/www.psychiatry.org/patients-families/anxietydisorders/what-are-anxiety-disorders

3. Baxter AJ, Scott KM, Vos T, Whiteford HA. Global prevalence of anxiety disorders: A systematic review and meta-regression. Psychol Med.2013;43(5):897-910. https://pubmed.ncbi.nlm.nih.gov/22781489/

4. Schwarts A. Anxiety, Worry, and Stress, Oh My: The Bugaboos of Modern Life. 2020. https://psychcentral.com/lib/anxiety-worry-and-stress-oh-my-the-bugaboos-of-modern-life/

5. Fond G, Loundou A, Hamdani N, Boukouaci W, Dargel A, Oliveira J, et al. Anxiety and depression comorbidities in irritable bowel syndrome (IBS): a systematic review and meta-analysis. Eur Arch Psychiatry Clin Neurosci.2014;264(8):651-60.

6. Haug TT, Mykletun A, Dahl AA. Are anxiety and depression related to gastrointestinal symptoms in the general population? Scand J Gastroenterol. 2002;37(3):294-8.

7. Grennan D. What Is a Pandemic? JAMA - J Am Med Assoc. 2019;321(9):910.

8. Moock M, De Carvalho Mello PMV. COVID-19 pandemic. Rev Bras Ter Intensiva. 2020;32(1):1.

9. Rubin GJ, Wessely S. The psychological effects of quarantining a city. BMJ Publishing Group; 2020. https://pubmed.ncbi.nlm.nih.gov/31992552/ 

10. Brooks SK, Webster RK, Smith LE, Woodland L, Wessely S, Greenberg N, et al. The psychological impact of quarantine and how to reduce it: rapid review of the evidence. Lancet. 2020;395(10227):912-20.

11. Ro JS, Lee JS, Kang SC, Jung HM. Worry experienced during the 2015 Middle East Respiratory Syndrome (MERS) pandemic in Korea. PLoS One. 2017;12(3):e0173234.

12. Goodwin R, Gaines SO, Myers L, Neto F. Initial psychological responses to swine flu. Int J Behav Med. 2011;18(2):88-92.

13. Reynolds DL, Garay JR, Deamond SL, Moran MK, Gold W, Styra R. Understanding, compliance and psychological impact of the SARS quarantine experience. Epidemiol Infect. 2008;136(7):997-1007.

14. Talevi D, Socci V, Carai M, Carnaghi G, Faleri S, Trebbi E, et al. Mental health outcomes of the covid-19 pandemic. Riv Psichiatr. 2020;55(3):137-44.

15. Wong TW, Gao Y, Tam WWS. Anxiety among university students during the SARS epidemic in Hong Kong. Stress Heal. 2007;23:31-5.

16. Zhang Y, Ma ZF. Impact of the COVID-19 pandemic on mental health and quality of life among local residents in Liaoning Province, China: A cross-sectional study. Int J Environ Res Public Health. 2020;17(7).

17. Sallam M, Dababseh D, Yaseen A, Al-Haidar A, Ababneh NA, Bakri FG, et al. Conspiracy beliefs are associated with lower knowledge and higher anxiety levels regarding covid-19 among students at the university of Jordan. Int $\mathrm{J}$ Environ Res Public Health. 2020;17(14):1-15.

\section{Correspondence:}

Luminița Hriscu,

Faculty of Veterinary Medicine, University of Agricultural Sciences and Veterinary Medicine "Ion Ionescu de la Brad" of Iasi, 3rd Mihail Sadoveanu Alley, Iași 700490, Romania,

lumidih@yahoo.com

Submission: 30 aug. 2020

Acceptance: 15 sep. 2020 



\title{
Gabapentin: an old anticonvulsivant with new potential, may reduce withdrawal symptoms and contribute to alcohol abstinence
}

\author{
Andreea Cătălina Rusu, Ana Caterina Cristofor, \\ George Cătălin Moroșan, Roxana Chiriță
}

Andreea Cătălina Rusu - M.D., junior psychiatrist, Socola Institute of Psychiatry, Iași

Ana Caterina Cristofor - M.D., PhD, Assistant Profesor, Grigore T. Popa University of Medicine and Pharmacy, Senior Psychiatrist Socola Institute of Psychiatry, Iaşi

George Cătălin Moroșan - M.D., junior psychiatrist, Socola Institute of Psychiatry, Iași

Roxana Chiriță - M.D. PhD, Professor of Psychiatry, Grigore T. Popa University of Medicine and Pharmacy, Senior Psychiatrist, Socola Institute of Psychiatry, Iași

\begin{abstract}
Alcohol abuse is one of the most common causes of substance dependence, representing an area of interest in continuous development. In terms of treatment, Gabapentin (GBP) has shown in published studies that it is an important therapeutic agent, especially in the case of alcohol withdrawal syndrome. The main objective of this article is to carry out a detailed evaluation of studies on the effectiveness of Gabapentin in the treatment of numerous mental disorders and also substance use disorders. Although it belongs to the category of anticonvulsants, it has been shown that Gabapentin has many therapeutic properties due to its mechanism of action involving the Gabaergic system. Thus, a new therapeutic vision in the treatment of abstinence and withdrawal syndrome is outlined.
\end{abstract}

KEYWORDS:

Gabapentin, alcohol use disorder, withdrawal symptoms, alcohol dependence, GABAergic system, abstinence.

\section{INTRODUCTION}

Alcohol use disorder (AUD) is a complex syndrome with multiple consequences in terms of socio-familial environment and health status. The World Health Organization estimates alcohol consumption is responsible for $5.1 \%$ of disease burden and for $5.9 \%$ of deaths, especially through liver cirrhosis, cancers and organ injuries (1).

ICD-10 (2) and DSM-IV (3) define alcohol related disorders by a cluster of psychological, somatic and behavioral symptoms. DSM-5 (4) has introduced 11 criteria for substance use disorders: (1) large 
\%

amounts consumed; (2) desire or inability to reduce use; (3) significant time spent obtaining, using, or recovering from use; failing to meet role obligations; (4) continued use despite problems; (5) reduced/ceased societal activities as a result of use; (6) results in physically dangerous situations; (7) cravings; (8) continued use despite insight to dangers; (9) tolerance develops;(10) withdrawal. Therefore 2 or 3 positive symptoms define a mild substance use disorder, 4 or 5 a moderate, and 6 or more a severe one.

This psychiatric problem with different degrees of severity is typically a chronic disorder characterized by compulsive heavy alcohol drinking (4). The occurrence of an acute withdrawal syndrome due to marked reduction in drinking is typically followed by a prolonged withdrawal phase with a high risk of relapse. Preventing relapsing after an acute withdrawal is a leading concern of alcoholism research (5).Gabapentin is an oral anticonvulsant with anxiolytic properties shown to have beneficial effects in a multitude of areas pertaining to optimal treatment of alcohol withdrawal, as well as alcohol dependence (6). A Cochrane review (2014) found evidence supporting the use of gabapentin for multiple health conditions (8) and pointed out that gabapentin has statistically positive effects in reducing alcohol consumption, but not in maintaining abstinence or reducing cravings (8). Gabapentin is a calcium channel GABAergic modulator that is widely recommended for neuropathic pain. Numerous medical studies have shown that gabapentin promotes a reduction of alcohol consumption in patients with depression or sleep disorders (7).

\section{PHARMACOKINETIC PROPERTIES}

A couple of FDA-approved anticonvulsants, including Gabapentin, that exert action on
GABA synthesis in the brain, have been studied for their ability in treating AUD and alcohol withdrawal syndrome (9). In individuals with alcohol related disorders, gabapentin significantly reduced alcohol consumption and cravings by improving the symptoms associated with the abstinence syndrome. The results highlight some findings that indicate that gabapentin is more effective in people with a history of alcohol withdrawal (9). Gabapentin is an aminoacid designed as a structural analogue of GABA (10) interacting with type 1 alpha-2-delta subunit of closed voltage calcium channels $(11,12)$ and selectively inhibits the influx of calcium (13) which results in decreased ability to reduce postsynaptic excitability(14). By blocking excitatory neurotransmission, gabapentin increases the concentration of GABA in the brain $(15,16)$. The mechanism involved in the psychoactive effects of GBP is not fully understood (17).

At concentrations up to $100 \mu \mathrm{M}$, gabapentin has no affinity for a number of receptor sites, including benzodiazepine, NMDA, alpha 1, alpha 2 or beta-adrenergic, glutamate, adenosine A1 or A2, cholinergic, dopamine D1 or D2, muscarinic or nicotinic, histamine $\mathrm{H} 1$, opiates $\mathrm{mu}$, serotonin $\mathrm{S} 1$ or $\mathrm{S} 2$, cannabinoid 1, delta or kappa. Gabapentin has not been shown to significantly influence the cellular action of dopamine, norepinephrine or serotonin. The half-life of gabapentin is 5-7 hours and no dose adjustment is required. Regarding the renal elimination, the clearance is directly proportional to the creatinine clearance, so that in patients with renal injury, the clearance of gabapentin is low, and dose adjustment is required.

The bioavailability of gabapentin is not directly related to the dose taken, and serum levels do not directly correlate with ingested doses (18). Gabapentin has minimal drug- 
drug interactions compared with previously studied anticonvulsants (carbamazepine, oxcarbamazepine, valproate, phenytoin, phenobarbital, topiramate) and a favourable safety profile (19).

\section{THERAPEUTIC EFFECTS OF GABAPENTIN}

Gabapentin has been indicated to treat many psychiatric and medical conditions, such as migraines, fibromyalgia and chronic pain syndromes. It is also widely prescribed for off-label psychiatric disorders $(19,20)$ originally developed to treat epileptic seizures, it is also recommended now to relieve neuropathic pain and restless legs syndrome. It is especially used as a first-line therapy for neuropathic pain as encountered in post-herpetic neuralgia, central neuropathic pain and diabetic neuropathy. Studies in rats and human subjects have shown an increase in GABA biosynthesis and in vitro neurotransmission, and the conclusion suggests that its modulation has the effect of sedating or calming the nervous system. Another mentioned mechanism involves the binding to the $\alpha 2 \delta-1$ subunit of calcium ion channels with voltage, which supports its analgesic effects.

Preclinical studies report the benefits of gabapentin related to seizures and anxiety. Moreover, gabapentin has been shown to be effective in reducing the excitability of the hippocampus, and in long-term administration has proven to have benefits in reducing recurrence and maintaining abstinence. This new potential makes him an attractive agent to explore these indications for managing alcohol withdrawal and addiction (21).

\section{GABAPENTIN AND WITHDRAWAL SYMPTOMS}

Benzodiazepines are considered the first choice for treating alcohol withdrawal, but
Gabapentin has been studied as a potential treatment, based on its modulatory action on brain inhibitory mechanisms (i.e., GABAergic).

One of the most common disorders of substance use is alcohol use disorder, including both alcohol consumption and addiction (22). Three drugs are approved and used in the treatment of alcohol abuse, and these are disulfiram, acamprosate and naltrexone, and a fourth drug called "nalmefene" is being approved. Numerous randomized studies have evaluated the role of GBP in the treatment of alcohol abuse and alcohol addiction. A number of 13 studies with a total of 807 patients $(23,24,25,26,27$, $28,29,30,31,32,33,34,35)$ were highlighted in the literature. Published studies illustrate the therapeutic effects of GBP in alcohol consumption disorders, by using relatively high doses of 1,200-3,200 mg GBP per day with a positive effect on cravings, depression, insomnia, withdrawal symptoms and in maintaining abstinence $(28,29)$. Sleep disorders such as insomnia are often associated with recurrence of consumption in alcohol-dependent patients, with rates ranging from $36-91 \%$. It can be hypothesized that treating insomnia in these patients may minimize recurrence rates. Studies show that GBP addresses the signs and symptoms of insomnia and recurrences in alcoholdependent patients (28).

Studies of alcohol-dependent animal models have shown that gabapentin decreases the amplitudes of GABA receptor-mediated postsynaptic inhibitory currents in the central amygdala (CeA), which results in a decrease in alcohol-induced dependence (36). Surprisingly, the effects of gabapentin seem to be identical to the effects of a corticotropinreleasing factor (CRF) antagonist (37, 38). These results suggest a significant GABA- 
$\mathrm{CRF}$ interaction in GABAergic neurotransmission that has the ability to adapt during the development of alcohol dependence and therefore GABA-CRF neuroadaptations induced by ethanol dependence may represent the differential effects of gabapentin observed in CeA (36).

Other mechanisms of action involve blocking the effects of thrombospondin on the alpha 2 delta type 1 subunit (38). Type 1 subunits have the ability to modulate closed-channel calcium channels, but can simultaneously perform functions independent of calcium. Astrocytes secrete a protein, named thrombospondin, with a role in promoting synaptogenesis, and gabapentin antagonizes the binding of thrombospondin to the alpha-2 delta subunit resulting in inhibition of excitatory synapse formation, action independent of the calcium channel (38). Previous research and studies have shown that alpha-2-delta type 1 subunits are regulated in reward-related regions by all major drugs of abuse, including alcohol (39).

A 12-week placebo-controlled dose study in 150 patients (40) indicated that Gabapentin has beneficial therapeutic effect on abstinence rates (placebo: 4.1\%; gabapentin $900 \mathrm{mg}$ : 11.1\%; gabapentin $1800 \mathrm{mg}: 17.0 \%$ ). In this study there were no side effects when taking the medicine. Used in combination with the benzodiazepine antagonist flumazenil, it did not show satisfactory results (41). The recent editorial by JAMA (Journal of the American Medical Association) (42) regarding current perspectives in alcohol pharmacotherapy highlights positive results from a 6-month study in a group of 348 patients who used either 1200mg of gabapentin, either placebo, with good results in the treatment of alcohol disorder.
Jonathan G. Leung's study was performed, at Mayo Clinic, Rochester, on 77 patients included in the primary evaluation of the gabapentin protocol, with a mean age of 48.7 +11.4 years, with the predominance of the male sex, respectively $72.7 \%$. The mean PAWSS( Prediction of Alcohol Withdrawal Severity Scale) scale for these patients was $4.7+1.4$, with $62(80.5 \%)$ patients with a PAWSS score of at least 4 indicating a high risk of complicated withdrawal. In this gabapentin protocol, $51.9 \%$ of patients received an average of $2.3+1.6 \mathrm{mg}$ lorazepam in the emergency department, and 4 of them received diazepam in doses between 10-20 mg (26). The results offer a new direction of treatment and include the use of gabapentin in the future, but this requires a well-established protocol, as well as further research into the safety of this anticonvulsant treatment (GBP) vs benzodiazepine (43).

Another study by JAMA Internal Medicine, on a group of 96 patients, divided into placebo-group and gabapentin treated group, conducted over a period of 16 weeks, showed that Gabapentin prevented alcohol consumption and promoted alcohol abstinence among patients. Subsequently, a marker of alcohol consumption was collected, the percentage of transferrin disialocarbohydrate was found to be deficient in the blood during treatment. Total abstinence was also higher in the gabapentin group - $18 \%$ compared to $4 \%$ in placebo group. However, about $33 \%$ of the participants in each group did not complete the process, a major limitation (44). Gabapentin was equally safe and tolerable, with no significant differences between the batch groups. These studies provide preliminary evidence that there may be a role for gabapentin on its own in the treatment of mild to moderate alcohol withdrawal syndrome. 


\section{CONCLUSIONS}

This article presents preliminary, but potentially convincing evidence collected from the scientific literature in support of Gabapentin therapy for alcohol withdrawal syndrome and abstinence. Early initiation of high-dose gabapentin was associated with a significant reduction in benzodiazepine requirements, but also a faster stabilization of symptoms related to discontinuation of alcohol consumption and a shorter hospital stay. This anticonvulsant is unlikely to replace benzodiazepines, the first line therapy, but may play an appropriate role and may be useful as monotherapy for milder cases with a lower risk of complications. The current indication for this drug remains for the treatment of alcohol withdrawal syndrome complicated with seizures, refractory forms, in association with benzodiazepines. Therefore, more clinical trials are needed to investigate the efficacy of this anticonvulsant, Gabapentin, in both psychiatric and substance use disorders, but also its grade of tolerance and safety.

\section{ACKNOWLEDGE AND DISCLOSURE}

The authors declare that they have no potential conflicts of interest to disclose.

\section{REFERENCES}

1. Global status report on alcohol and health 2014.2017.World Health Organization.

2. World Health Organization. International classification of diseases, 10th revision (ICD-10). 2010.

3. American Psychiatric Association. Diagnostic and statistical manual of mental disorders. 4th ed. Washington (DC): American Psychiatric Publishing; 2000. Text Revision

4. American Psychiatric Association. Diagnostic and statistical manual of mental disorders. 5th ed. Arlington (VA): American Psychiatric Publishing; 2013.

5. Gordis E. Relapse and craving: a commentary. Alcohol Alert 1989;6:3

6. Nina Vadiei, Tawny L. Smith, Amy E. Walton, Kimberly L. Kjome ' Impact of Gabapentin Adjunct use with Benzodiazepines for the Treatment of Alcohol Withdrawal in a Psychiatric Hospital ' 2019 Feb 15; 49(1): 17-27.

7. Mason B.J., Quello S., Shadan F. Gabapentin for the treatment of alcohol use disorder. 2017.

8. Pani P.P., Trogu E., Pacini M. et al. Anticonvulsants for alcohol dependence. Cochrane Database Syst Rev.2014;2:CD008544.

9. Verplaetse T.L., McKee S.A., Petrakis I.L. Pharmacotherapy for Co-Occurring Alcohol Use Disorder and PostTraumatic Stress Disorder Targeting the Opioidergic, Noradrenergic, Serotonergic, and GABAergic/Glutamatergic Systems. 2018.

10. Sills G.J. The mechanisms of action of gabapentin and pregabalin. Curr Opin Pharmacol. 2006;6(1):108-13.

11. Xiao W., Boroujerdi A., Bennet G.J., Luo Z.D. Chemotherapy-evoked painful peripheral neuropathy: analgesic effects of gabapentin and effects on expression of the alpha-s-delta-1 calcium channel subunit. Neuroscience. 2007;144(2):714-20.

12. Uchitel O.D., Di Giulmi M.N., Urbano F.J., Gonzalez-Inchauspe C. Acute modulation of calcium currents and synaptic transmission by gabapentinoids. Channels.2010;4(6):490- 6.

13. Fink K., Meder W., Dooley D.J., Göthert M. Inhibition of neuronal Ca2+ influx by gabapentin and subsequent reduction of neurotransmitter release from rat neocortical slices. Br J Pharmacol. 2000;130(4):900-06

14. Cunningham MO, Woodhall GL, Thompson SE, et al. Dual effects of gabapentin and pregabalin on glutamate release at rat entorhinal synapses in vitro. Eur J Neurosci 2004;20(6):1566-76

15. Taylor CP, Gee NS, Su TZ, et al. A summary of mechanistic hypotheses of gabapentin pharmacology. Epilepsy Res 1998;29(3):233-49

16. Götz E., Feuerstein T.J., Lais A., Meyer D.K. Effects of gabapentin on release of gamma- aminobutyric acid from slices of rat neostriatum. Arzneimittelforschung.1993;43(6):636-8.

17. Wallach J.D., Ross J.S. Gabapentin approvals, off-label use, and lessons for postmarketing evaluation efforts. JAMA .2018;319(8):776-8.

18. Stewart B.H., Kugler A.R., Thompson P.R., Bockbrader H.N. A storable transport mechanism in the intestinal absorption of gabapentin is the underlying cause of the lack of proportionality between increasing dose and drug levels in plasma. Pharm Res.1993;10:276-81

19. Perry E.C. Inpatient management to acute alcohol withdrawal syndrome. CNS Drugs.2014;28(5):401-410. 


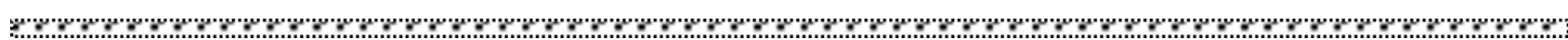
20. Houghton K.T., Forrest A., Awad A., Atkinson L.Z., Stockton S., Harrison P.J. et al. Biological rationale and potential clinical use of gabapentin and pregabalin in bipolar disorder, insomnia and anxiety: protocol for a systematic review and meta-analysis. BMJ Open.2017;7(3).

21. Leung J.G., Hall-Flavin D., Nelson S., Kristen A., Schmidt, Schak K.M. The Role of Gabapentin in the Management of Alcohol Withdrawal and Dependence. 2015;1-10.

22. Elkins R.L., Richards T.L., Nielsen R., Repass R., Stahlbrandt H., Hoffman H.G. The neurobiological mechanism of chemical aversion (emetic) therapy for alcohol use disorder: an fMRI study. Front Behav Neurosci.2017;11:182.

23. Anton R.F., Myrick H., Baros A.M., Latham P.K., Randall P.K., Wright T.M. et al. Efficacy of a combination of flumazenil and gabapentin in the treatment of alcohol dependence: relationship to alcohol withdrawal symptoms. J Clin Psychopharmacol.2009; 29(4):334-42.

24. Anton R.F., Myrick H., Wright T.M., Latham P.K., Baros A.M., Waid L.R. et al. Gabapentin combined with naltrexone for the treatment of alcohol dependence. Am J Psychiatry.2011;168(7):709-17.

25. Bisaga A., Evans S.M. The acute effects of gabapentin in combination with alcohol in heavy drinkers. Drug Alcohol Depend.2006; 83(1):25-32.

26. Bonnet U., Banger M., Leweke F.M., Specka M., Müller B.W., Hashemi T et al. Treatment of acute alcohol withdrawal with gabapentin: results from a controlled two-centre trial. J Clin Psychopharmacol.2003;23(5):514-9.

27. Bonnet U., Hamzavi-Abedi R., Specka M., Wiltfang J., Lieb B., Scherbaum N. An open trial of gabapentin in acute alcohol withdrawal using an oral loading protocol. Alcohol Alcohol.2009;45(2):143-5.

28. Brower K.J., Myra Kim H., Strobbe S., Karam-Hage M.A., Consens F., Zucker R.A. A randomized double-blind pilot trial of gabapentin versus placebo to treat alcohol dependence and comorbid insomnia. Alcohol ClinExp Res.2008;32(8):1429-38.

29. Furieri F.A., Nakamura-Palacios E.M. Gabapentin reduces alcohol consumption and craving: a randomized, double-blind, placebo-controlled trial. J Clin Psychiatry.2007; 68(11):1691-700.

30. Mason B.J., Quello S., Goodell V. et al. Gabapentin treatment for alcohol dependence: a randomized clinical trial. JAMA Intern Med. 2014;174(1):70-77.

31. Mason B.J., Quello S., Goodell V., Shadan F., Kyle M., Begovic A. Gabapentin treatment for alcohol dependence: a randomized clinical trial. JAMA Int Med.2014;174(1):70-7.

32. Myrick H., Anton R., Voronin K., Wang W., Henderson S. A double-blind evaluation of gabapentin on alcohol effects and drinking in a clinical laboratory paradigm. Alcohol ClinExp Res.2007;31(2):221-7.

33. Myrick H., Malcolm R., Randall P.K., Boyle E., Anton R.F., Becker H.C. et al. A double-blind trial of gabapentin versus lorazepam in the treatment of alcohol withdrawal. Alcohol ClinExp Res.2009;33(9):1582-8.

34. Stock C.J., Carpenter L., Ying J., Greene T. Gabapentin versus chlordiazepoxide for outpatient alcohol detoxification treatment. Ann Pharmacother.2013;47(7-8):961-9.

35. Trevisan L.A., Ralevski E., Keegan K., Oville A., Vuppalapati D., Gonzalez G. et al. Alcohol detoxification and relapse prevention using valproic acid versus gabapentin in alcohol-dependent patients. Addict Disord Their Treat.2008;7(3):119-28.

36. Roberto M., Gilpin N.W., O’Dell L.E. et al. Cellular and behavioral interactions of gabapentin with alcohol dependence. J Neurosci. 2008;28(22):5762-71.

37. Roberto M., Cruz M.T., Gilpin N.W. et al. Corticotropin releasing factor-induced amygdala gamma-aminobutyric acid release plays a key role in alcohol dependence. Biol Psychiatry. 2010;67(9):831-9.

38. Eroglu C., Allen N.J., Susman M.W. et al. Gabapentin receptor alpha2delta-1 is a neuronal thrombospondin receptor responsible for excitatory CNS synaptogenesis. Cell. 2009;139(2):380- 92.

39. Katsura M., Shibasaki M., Hayashida S. et al. Increase in expression of alpha1 and alpha2/delta1 subunits of Ltype high voltage-gated calcium channels after sustained ethanol exposure in cerebral cortical neurons. J Pharmacol Sci.2006;102(2):221-30.

40. Mason B.J., Light J.M., Williams L.D., Drobes D.J. Proof-of-concept human laboratory study for protracted abstinence in alcohol dependence: effects of gabapentin. Addict Biol.2009;14(1):73-83.

41. Anton R.F., Myrick H., Baros A.M. et al. Efficacy of a combination of flumazenil and gabapentin in the treatment of alcohol dependence: relationship to alcohol withdrawal symptoms. J Clin Psychopharmacol. 2009;29(4):334-342.

42. Lyon J. More treatments on deck for alcohol use disorder. JAMA. 2017;13:317(22):2267-2269. 
Bulletin of Integrative Psychiatry O New Series OSeptember 2020 ○ Year XXVI ONo. 3 (86)/77

Fyon 43. Jonathan G.L., Rakocevic D., Nicholas D. Allen, Elliot M. Handler, Bruno A. Perossa et al. Use of a gabapentin protocol for the management of alcohol withdrawal: a preliminary experience expanding from the consultation/liaison psychiatry service. A gabapentin protocol for alcohol withdrawal.

https://jamanetwork.com/ by a Uppsala University User on 05/30/2020

\section{Correspondence:}

Ana Caterina Cristofor,

M.D., PhD, Assistant Profesor, Grigore T. Popa University of Medicine and Pharmacy,

Psychiatrist, Socola Institute of Psychiatry, Iași

Submission:22 feb 2020

Acceptanec: 17 apr 2020 



\title{
A review on brain-derived neurotrophic factor and its role in depression and anxiety
}

\author{
Vlad Teodor Iacob, Matei Palimariciuc, \\ Vasile Chiriță, Roxana Chiriță
}

\begin{abstract}
Vlad Teodor Iacob - M.D., junior psychiatrist, "Socola” Institute of Psychiatry, Iași, Romania Matei Palimariciuc - M.D., PhD student, Assistant professor, "Grigore T. Popa" University of Medicine and Pharmacy Iași, Romania, junior psychiatrist "Socola" Institute of Psychiatry, Iași, Romania Vasile Chiriță - Professor, Academician, PhD, Psychiatrist, Honorary Member of Romanian Academy

Roxana Chiriță - M.D. PhD, Professor of Psychiatry "Grigore T. Popa" University of Medicine and Pharmacy Iași, Romania, senior psychiatrist "Socola" Institute of Psychiatry, Iași, Romania
\end{abstract}

\begin{abstract}
Brain-derived neurotrophic factor (BDNF) is a well-deliberated growth factor that serves many purposes throughout the central nervous system (CNS) and has been implicated in various psychiatric and neurological disorders. This article reviews the current understanding and future directions in BDNF-related research in the CNS, with an emphasis on its relation to depression and anxiety-related disorders.
\end{abstract}

\section{KEYWORDS:}

BDNF, neurotrophin, depression, anxiety.

\section{INTRODUCTION}

Mood and anxiety disorders are arguably amongst the most disabling of all medical disorders. They frequently appear together and early in life, run a chronic course and adversely affect the prognosis of other medical illnesses (1). Understanding the pathophysiology of these disorders is crucial in the effort to better predict or prevent their onset, to develop new treatments, and to better adapt each treatment to each individual (2). Brain-derived neurotrophic factor (BDNF) is a well-studied growth factor that

serves many functions within the central nervous system (CNS) and has been implicated in several psychiatric disorders, such as autism, intellectual disability, schizophrenia, and the development of anxiety and mood disorders, such as depression and its treatment (3).

BDNF FROM A MORPHOFUNCTIONAL STANDPOINT 
BDNF is a member of the neurotrophin family, including nerve growth factor (NGF), neurotrophin-3 (NT3) and neurotrophin-4 (NT4). For almost 40 years, research has been conducted on BDNF, and while it has been fruitful in many fields, we have yet to fully understand it and use it to its full potential. Through dendritic arborization and synaptic consolidation, BDNF mediates, amongst others, neuronal development, synaptic plasticity and long-term potentiation in the CNS (4, 5, 6, 7, 8). BDNF also influences learning and memory $(9,10,11)$, and modulates hypothalamic metabolic function $(12,13)$. It is secreted by neurons and peripheral cells, including leukocytes (14), endothelial cells (15), and platelets $(16,17)$, and passes the blood-brain barrier (18), albeit poorly (4).

Although the matter is still debatable, it seems that there are two forms of extracellular BDNF: proBDNF and mature BDNF (mBDNF). After its release from neurons, the pro-domain of pro-BDNF is proteolytically cleaved by plasmin or metalloproteinases, resulting mBDNF $(19,20)$. Recent studies suggest that the mature form of BDNF is predominant and of greatest physiological relevance in the adult brain (21.22). Regarding receptor binding affinities, BDNF binds with high specificity to the tropomyosin receptor kinase $\mathrm{B}$ (TrkB) and the low-affinity neurotrophin receptor p75 (p75NTR). While TrkB is widely expressed throughout the adult brain, including the cortex, hippocampus, and multiple brainstem and spinal cord nuclei (23), the expression of p75NTR is restricted in adulthood to basal forebrain cholinergic neurons and relatively few cortical neurons (24). Furthermore, mBDNF binds with great affinity to TrkB, whereas pro-BDNF binds with a higher affinity to p75NTR (19). This distinction in BDNF isoform binding is relevant because TrkB predominantly supports neuronal survival and expression of several genes (25), while separate p75NTR activations result in pro-apoptotic signalling (26).

\section{BDNF AND ITS FUNCTION IN MOOD AND ANXIETY DISORDERS}

A number of individual studies and later meta-analyses in the last few decades have reported perturbations in BDNF expression in both depression and anxiety, and its return to normal levels after various treatments for depression, including antidepressants $(27,28$, 29).Reduced BDNF levels have been found in the postmortem samples of brains of depressed patients and of suicide victims, many of which suffer from severe depression (29). Furthermore, levels of its receptor, TrkB, have also been reported to be downregulated in the brain of depressed patients (30) and the levels of active, phosphorylated form of TrkB are reduced in suicide victims (31), indicating reduced BDNF signalling through TrkB in depression. On the other hand, increased levels of mRNA for the p75NTR neurotrophin receptor have been reported in the brains of suicide victims (32). In the adult brain, upon neuronal trauma, p75NTR expression is increased in cortical and hippocampal neurons and as previously mentioned, p75NTR mediates degeneration and death of injured neurons (26, 29). Increase in p75NTR levels in suicide victims suggests that severe psychological stress, such as leading to suicide, may act similar to brain trauma. p75NTR signalling may promote synaptic depression and pruning and other "antitrophic" effects that could at least partially explain the pathophysiology of depression (29).

Regarding treatment, conventional antidepressants, such as selective serotonin reuptake inhibitors (SSRIs) or tricyclic antidepressants (TCAs), mediate their 
Bulletin of Integrative Psychiatry O New Series OSeptember 2020 ○ Year XXVI ONo. 3 (86)/81

\%

antidepressant effect by modulating extracellular levels of monoamines, mainly serotonin or norepinephrine. It is thought that these drugs enhance the extracellular levels of monoamines rather quickly, within hours.

However, the antidepressant response is delayed and typically requires weeks of treatment before the onset of therapeutic actions $(33,34)$. Therefore, other mechanisms relating to the enhancement of extracellular level of monoamines are believed to mediate the antidepressant response (4). In this context, BDNF has been linked to the mechanism of action of antidepressants. The first studies to implicate BDNF in antidepressant responses reported that conventional antidepressant drugs, as well as electroconvulsive therapy (ECT), enhanced BDNF and TrkB mRNA expression in the hippocampus and cortical regions in a time span similar to the onset of therapeutic actions of antidepressants. Subsequent studies took a more direct approach in their research by infusing BDNF protein directly into the midbrain of rodents, observing an antidepressant-like effect (35). Further work showed that a single bilateral infusion of a low dose of BDNF directly into the dentate gyrus (DG) or CA3, but not CA1 region of the hippocampus was sufficient to induce an antidepressant-like effect within three days (5), suggesting that these regions may be critical to understanding the antidepressant effect in connection to BDNF. Moreover, this latter study noted that BDNF produced an antidepressant effect for up to 10 days after infusion, well past the time frame for the degradation of the protein, suggesting that BDNF may be triggering a sustained plasticity mechanism to mediate the long-term antidepressant effects. Its theory states that the antidepressant effects of BDNF are mediated by TrkB activation of mitogenactivated protein kinase kinase (MEK), acting through the extracellular signal-regulated pathway (5). In a separate study, peripheral, subcutaneous injections of BDNF were reported to produce antidepressant and anxiolytic behavioural responses in animal models, prevent depression-related effects of chronic stress, and an increase to the survival rate of newborn neurons in the hippocampus and prefrontal cortex (36). However, the exact mechanism of this conclusion remains to be explained, as BDNF is poorly transported across the blood-brain barrier, leaving it unclear as to how peripheral administration mediates the aforementioned effects (4).

Further evidence supporting the involvement of BDNF signalling due to the effects of antidepressant drugs has emerged from studies examining the high-affinity BDNF receptor, TrkB. Antidepressants require functional $\operatorname{TrkB}$ receptors to mediate antidepressant-like responses in preclinical models (37, 38). Interestingly, long-term antidepressant treatment increases the phosphorylation of the TrkB receptor, thus increasing its activity in the hippocampus and anterior cingulate cortex suggesting that BDNF-TrkB signalling in these regions may be of great importance in mediating antidepressant effects (37). The lastmentioned study has also concluded that conventional antidepressants necessitate monoamines to affect BDNF intracellular signalling as monoamine depletion prevented the antidepressant-induced activation of TrkB (37).

While there is strong data that supports the fact that BDNF in the hippocampus and prefrontal cortex is involved in mediating antidepressant responses, other brain regions have also been involved in antidepressant responses. For example, BDNF signalling in the ventral tegmental area (VTA) to nucleus accumbens pathway appears to be producing 
effects that are in many ways opposite to the effects of BDNF in the hippocampus (39). BDNF injected directly into the VTA induces depression-like behaviour and, contrarily, blocking BDNF signalling in the nucleus accumbens, a brain region which receives afferents from the VTA, induces an antidepressant-like effect (40). BDNF signalling in this region has also been found to be essential for the development of the molecular and behavioural manifestations of stress $(41,42)$, hence further proving the involvement of BDNF in the mesolimbic pathway in the expression of at least a subgroup of the depressive symptoms. Another brain region that has been implicated in mood disorders is the lateral habenula, although it remains unclear if BDNF in this region has any connection to antidepressant responses or depression-related behaviour (4). As a result, BDNF-TrkB signalling in the hippocampus seems to be a vital component of antidepressant responses to clinically used antidepressants, at least in preclinical animal models. Nonetheless, the involvement of the hippocampus does not exclude the involvement of other brain regions, as, without a doubt, distinct neural circuits are required for antidepressant responses. Although, the requirement for BDNF in the hippocampus in mediating antidepressant responses is the first step to fully understand the neural circuitry involved in this process (4).

\section{THERAPEUTIC POTENTIAL AND THE DIFFICULTY OF DELIVERY TO THE CNS}

Even amongst the nervous system growth factors, BDNF has emerged as a leading candidate of interest as a potential neuroprotective and functionally restorative treatment in various psychiatric and neurological disorders, including amyotrophic lateral sclerosis (ALS), peripheral neuropathy,
Alzheimer's disease and Parkinson's disease (28).

As previously stated, a considerable number of studies reported alterations in BDNF expression in depressive and anxiety-related disorders. Patients who suffer from depression exhibit specific reductions in hippocampal volume, and in hippocampal and serum BDNF levels. Antidepressants reverse reductions in hippocampal volume, and several antidepressant therapies have been reported to normalize serum BDNF levels. Given the fact that BDNF is important in maintaining hippocampal dendritic morphology and synaptic function (4-8), it may contribute to the reversal of hippocampal atrophy due to the effects of antidepressants, although other factors may also be likely involved, such as activity and exercise (28).

The main challenge in the field of growth factor therapy is the drug delivery system to the CNS. BDNF is a reasonably sized and charged protein, which accounts for the fact that a minimal amount of BDNF crosses the blood-brain barrier via peripheral administration (28). In order to reach neurons from the brain or spinal cord, BDNF must be administered directly into the CNS. However, when administered intraventricularly or intrathecally, BDNF infusion results in little penetration of the brain or spinal cord parenchyma beyond the most superficial layers (43). Moreover, when growth factors do "spread" through the nervous system after intrathecal administration, they cause intolerable adverse effects over long periods of time. These adverse effects include weight loss, dysaesthesia and migration and/or proliferation of Schwann cells in the subpial space (44-47). Another important observation in the study of growth factor therapies is the need for extended periods of treatment, depending on which disorder is being treated. 
Thus, the ideal delivery system of growth factors to the nervous system must achieve concentrations at precise sites to limit the spread to distant sites and, as such, prevent adverse effects as much as possible. Two potential approaches are in the running for a safe delivery system of growth factors such as BDNF to the nervous system: intraparenchymal protein infusion (48-50) and gene delivery using viral vectors (51), both of which require further development (28).

\section{DISCUSSION}

While there is still much controversy over the relation between BDNF and anxiety in animals and humans (52-56), there is a vast amount of knowledge and evidence that strongly implicates BDNF in the pathophysiology of depression, and many other medical conditions. These findings lead to several potentially translational possibilities. To start with, BDNF has great potential for its use as a biomarker, for diagnosing mood disorders and monitoring their treatment response. There is also the therapeutic potential of BDNF for treating various psychiatric and neurological disorders, with ongoing research fiercely trying to solve the drug delivery system challenge through different approaches: by enhancing the accuracy of vector and growth factor targeting to the brain using real-time MRI during treatment (57); by improving gene therapy vectors to achieve persistent, safe and regulatable growth factor expression in the CNS (58); and by developing peptide mimetics of growth factors with specificity for degenerating cell systems (59).

Nonetheless, we still have a long way to go until we can fully understand its mechanism of action and apply that information to successfully construct and customise new treatment options. There is a great deal of work needed in delineating the regional specificity of BDNF TrkB signalling, with the hippocampus and its involvement in mediating antidepressant responses being the first step for fully understanding the neural circuitry involved in this process.

\section{CONCLUSIONS}

The data reviewed above suggests a close relationship between BDNF signalling, neuronal plasticity and depression and its treatment, with ongoing research focused on applying BDNF as a viable biomarker and treatment option.

\section{ACKNOWLEDGEMENTS AND DISCLOSURES}

The authors declare that they have no potential conflict of interest to disclose.

\section{REFERENCES}

1. Kalaria RN, Maestre GE, Arizaga R, Friedland RP, et al.2008.Alzheimer's disease and vascular dementia in developing countries: prevalence, management, and risk factors. Lancet Neurol 7:812-826.

2. Guest PC, Reviews on Biomarker Studies in Psychiatric and Neurodegenerative Disorders. Department of Biochemistry and Tissue Biology Institute of Biology University of Campinas (UNICAMP) Campinas, Brazil.

3. Autry AE, Monteggia LM. Brain-derived neurotrophic factor and neuro-psychiatric disorders. Pharmacol. Rev. 2012; 64(2):238-258.

4. Björkholm C, Monteggia LM. BDNF-a key transducer of antidepressant effects.Neuropharmacology. 2016;102:7279.

5. Shirayama Y, Chen AC, Nakagawa S, Russell DS, et al. Brain-derived neurotrophic factor produces antidepressant effects in behavioral models of depression. J. Neurosci.2002; 22(8):3251-3261.

6. Polyakova M, Stuke K, Schuemberg K, Mueller K, et al. BDNF as a biomarker for successful treatment of mood disorders: a systematic \& quantitative meta-analysis.J Affect Disord. 2015;174:432-440. 


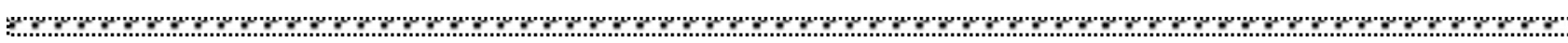
7. Figurov A., Pozzo-Miller L. D., Olafsson, P., et al. Regulation of synaptic responses to high-frequency stimulation and LTP by neurotrophins in the hippocampus. Nature. 1996; 381, 706-709.

8. Kang H., Schuman EM. Long-lasting neurotrophin-induced enhancement of synaptic transmission in the adult hippocampus. Science.1995;267, 1658-1662.

9. Yamada K, Mizuno M, Nabeshima T. Role for brain-derived neurotrophic factor in learning and memory. Life Sci. 2002;70, 735-744.

10. Egan MF, Kojima M, Callicott JH, et al. The BDNF val66met polymorphism affects activity-dependent secretion of BDNF and human memory and hippocampal function. Cell. 2003;112, 257-269.

11. Cathomas F, Vogler C, Euler-Sigmund JC, de Quervain DJ, et al. Fine-mapping of the brain-derived neurotrophic factor (BDNF) gene supports an association of the Val66Met polymorphism with episodic memory. Int. J. Neuropsychopharmacol. 2010;13, 975-980.

12. Xu B, Goulding EH, Zang K, et al. Brain-derived neurotrophic factor regulates energy balance downstream of melanocortin-4 receptor. Nat Neurosci. 2003;6(7):736-742.

13. Cao L, Lin EJ, Cahill MC, Wang C, Liu X, During MJ. Molecular therapy of obesity and diabetes by a physiological autoregulatory approach. Nat Med. 2009;15(4):447-454.

14. Edling AE, Nanavati T, Johnson JM, Tuohy VK. Human and murine lymphocyte neurotrophin expression is confined to B cells. J Neurosci Res. 2004;77(5):709-717.

15. Nakahashi T, Fujimura H, Altar CA, et al. Vascular endothelial cells synthesize and secrete brain-derived neurotrophic factor. FEBS Lett. 2000;470(2):113-117.

16. Yamamoto H, Gurney ME. Human platelets contain brain-derived neurotrophic factor. J Neurosci. 1990;10(11):3469-3478.

17. Fujimura H, Altar CA, Chen R, et al. Brain-derived neurotrophic factor is stored in human platelets and released by agonist stimulation. Thromb Haemost. 2002;87(4):728-734.

18. Pan W, Banks WA, Fasold MB, Bluth J, et al. Transport of brain-derived neurotrophic factor across the blood-brain barrier. Neuropharmacology.1998;37(12):1553-1561.

19. Lee R, Kermani P, Teng KK, Hempstead BL. Regulation of cell survival by secreted proneurotrophins. Science. 2001;294(5548):1945-1948.

20. Nikoletopoulou V, Lickert H, Frade JM, et al. Neurotrophin receptors TrkA and TrkC cause neuronal death whereas TrkB does not. Nature. 2010;467(7311):59-63.

21. Rauskolb S, Zagrebelsky M, Dreznjak A, et al. Global deprivation of brain-derived neurotrophic factor in the CNS reveals an area-specific requirement for dendritic growth. J Neurosci. 2010;30(5):1739-1749.

22. Matsumoto T, Rauskolb S, Polack M, et al. Biosynthesis and processing of endogenous BDNF: CNS neurons store and secrete BDNF, not pro-BDNF. Nat Neurosci. 2008;11(2):131-133.

23. Yan Q, Radeke MJ, Matheson CR, Talvenheimo J, Welcher AA, Feinstein SC. Immunocytochemical localization of TrkB in the central nervous system of the adult rat J Comp Neurol. 1997;378(1):135-157.

24. Lu B, Buck CR, Dreyfus CF, Black IB. Expression of NGF and NGF receptor mRNAs in the developing brain: evidence for local delivery and action of NGF. Exp Neurol. 1989;104(3):191-199.

25. Kaplan DR, Miller FD. Neurotrophin signal transduction in the nervous system. Curr Opin Neurobiol. 2000;10(3):381-391.

26. Koshimizu H, Hazama S, Hara T, Ogura A, et al. Distinct signaling pathways of precursor BDNF and mature BDNF in cultured cerebellar granule neurons. Neurosci Lett. 2010;473(3):229-232.

27. Martinowich K, Manji H, Lu B. New insights into BDNF function in depression and anxiety. Nat Neurosci. 2007;10(9):1089-1093.

28. Nagahara AH, Tuszynski MH. Potential therapeutic uses of BDNF in neurological and psychiatric disorders. Nat Rev Drug Discov. 2011;10(3):209-219.

29. Castrén E, Kojima M. Brain-derived neurotrophic factor in mood disorders and antidepressant treatments. Neurobiol Dis. 2017;97(Pt B):119-126.

30. Tripp A, Oh H, Guilloux JP, Martinowich K, et al. Brain-derived neurotrophic factor signaling and subgenual anterior cingulate cortex dysfunction in major depressive disorder. Am J Psychiatry. 2012;169(11):1194-1202.

31. Dwivedi Y, Rizavi HS, Conley RR, Roberts RC, Tamminga CA, Pandey GN. Altered gene expression of brainderived neurotrophic factor and receptor tyrosine kinase $\mathrm{B}$ in postmortem brain of suicide subjects. Arch Gen Psychiatry. 2003;60(8):804-815.

32. Dwivedi Y, Rizavi HS, Zhang H, et al. Neurotrophin receptor activation and expression in human postmortem brain: effect of suicide.Biol Psychiatry. 2009;65(4):319-328. 
(2) 33. Trivedi MH, Rush AJ, Wisniewski SR, et al. Evaluation of outcomes with citalopram for depression using measurement-based care in STAR*D: implications for clinical practice. Am J Psychiatry. 2006;163(1):28-40.

34. Stahl SM. Stahl's essential psychopharmacology: Prescriber's Guide, Sixth Edition. Cambridge: Cambridge University Press.

35. Siuciak JA, Lewis DR, Wiegand SJ, Lindsay RM. Antidepressant-like effect of brain-derived neurotrophic factor (BDNF). Pharmacol Biochem Behav. 1997;56(1):131-137.

36. Schmidt HD, Duman RS. Peripheral BDNF produces antidepressant-like effects in cellular and behavioral models. Neuropsychopharmacology. 2010;35(12):2378-2391.

37. Rantamäki T, Hendolin P, Kankaanpää A, et al. Pharmacologically diverse antidepressants rapidly activate brainderived neurotrophic factor receptor TrkB and induce phospholipase-Cgamma signaling pathways in mouse brain. Neuropsychopharmacology. 2007;32(10):2152-2162.

38. Saarelainen T, Hendolin P, Lucas G, et al. Activation of the TrkB neurotrophin receptor is induced by antidepressant drugs and is required for antidepressant-induced behavioral effects. J Neurosci. 2003;23(1):349-357.

39. Nestler EJ, Carlezon WA Jr. The mesolimbic dopamine reward circuit in depression. Biol Psychiatry. 2006;59(12):1151-1159.

40. Eisch AJ, Bolaños CA, de Wit J, et al. Brain-derived neurotrophic factor in the ventral midbrain-nucleus accumbens pathway: a role in depression. Biol Psychiatry. 2003;54(10):994-1005.

41. Berton O, McClung CA, Dileone RJ, et al. Essential role of BDNF in the mesolimbic dopamine pathway in social defeat stress. Science. 2006;311(5762):864-868.

42. Lippmann M, Bress A, Nemeroff CB, Plotsky PM, Monteggia LM. Long-term behavioural and molecular alterations associated with maternal separation in rats. Eur J Neurosci. 2007;25(10):3091-3098.

43. Ankeny DP, McTigue DM, Guan Z, et al. Pegylated brain-derived neurotrophic factor shows improved distribution into the spinal cord and stimulates locomotor activity and morphological changes after injury. Exp Neurol. 2001;170(1):85-100.

44. Winkler J, Ramirez GA, Kuhn HG, et al. Reversible Schwann cell hyperplasia and sprouting of sensory and sympathetic neurites after intraventricular administration of nerve growth factor. Ann Neurol. 1997;41(1):82-93.

45. Williams LR. Hypophagia is induced by intracerebroventricular administration of nerve growth factor. Exp Neurol. 1991;113(1):31-37.

46. Eriksdotter Jönhagen M, Nordberg A, Amberla K, et al. Intracerebroventricular infusion of nerve growth factor in three patients with Alzheimer's disease. Dement Geriatr Cogn Disord. 1998;9(5):246-257.

47. Isaacson LG, Saffran BN, Crutcher KA. Intracerebral NGF infusion induces hyperinnervation of cerebral blood vessels. Neurobiol Aging. 1990;11(1):51-55.

48. Kordower JH, Palfi S, Chen EY, et al. Clinicopathological findings following intraventricular glial-derived neurotrophic factor treatment in a patient with Parkinson's disease. Ann Neurol. 1999;46(3):419-424.

49. Nutt JG, Burchiel KJ, Comella CL, et al. Randomized, double-blind trial of glial cell line-derived neurotrophic factor (GDNF) in PD. Neurology. 2003;60(1):69-73.

50. Lang AE, Gill S, Patel NK, et al. Randomized controlled trial of intraputamenal glial cell line-derived neurotrophic factor infusion in Parkinson disease. Ann Neurol. 2006;59(3):459-466.

51. Gasmi M, Herzog CD, Brandon EP, et al. Striatal delivery of neurturin by CERE-120, an AAV2 vector for the treatment of dopaminergic neuron degeneration in Parkinson's disease. Mol Ther. 2007;15(1):62-68.

52. Rieder R, Wisniewski PJ, Alderman BL, Campbell SC. Microbes and mental health: A review.Brain Behav Immun. 2017;66:9-17.

53. Cirulli F, Berry A, Chiarotti F, Alleva E. Intrahippocampal administration of BDNF in adult rats affects short-term behavioral plasticity in the Morris water maze and performance in the elevated plus-maze. Hippocampus. 2004;14(7):802-807.

54. Rios M, Fan G, Fekete C, et al. Conditional deletion of brain-derived neurotrophic factor in the postnatal brain leads to obesity and hyperactivity. Mol Endocrinol. 2001;15(10):1748-1757.

55. Koponen E, Võikar V, Riekki R, et al. Transgenic mice overexpressing the full-length neurotrophin receptor trkB exhibit increased activation of the trkB-PLCgamma pathway, reduced anxiety, and facilitated learning. Mol Cell Neurosci. 2004;26(1):166-181.

56. Lang UE, Hellweg R, Kalus P, et al. Association of a functional BDNF polymorphism and anxiety-related personality traits. Psychopharmacology (Berl). 2005;180(1):95-99. 

convection-enhanced delivery of AAV2 vectors [published correction appears in Mol Ther. 2012 Feb;20(2):468. Salegio, Ernesto Aguilar . Mol Ther. 2010;18(8):1490-1495.

58. Goverdhana S, Puntel M, Xiong W, et al. Regulatable gene expression systems for gene therapy applications: progress and future challenges. Mol Ther. 2005;12(2):189-211.

59. Massa SM, Yang T, Xie Y, et al. Small molecule BDNF mimetics activate TrkB signaling and prevent neuronal degeneration in rodents. J Clin Invest. 2010;120(5):1774-1785.

\section{Correspondence:}

Matei Palimariciuc,

M.D., PhD Student, Assistant professor, "Grigore T.Popa” University of Medicine and Pharmacy, Iași; junior psychiatrist, matei.palimariciuc@umfiasi.ro

Submission: 01 aug 2020

Acceptance: 9 sep 2020 


\title{
Hormonal effects of estrogen and progesterone in postpartum depression
}

\author{
Roxana Mihaela Barbu, Cristina-Maria Gavrilescu, Elena Cojocaru, \\ Răducu Ionuț Popescu, Daniela Ababei, Walther Bild
}

\begin{abstract}
Roxana Mihaela Barbu - MD, PhD, Department of Physiology, University of Medicine and Pharmacy „Grigore T. Popa”, Iași, Specialist in Clinical Pharmacology, Romania.

Cristina-Maria Gavrilescu - Lecturer, MD, PhD, „Grigore T. Popa” University of Medicine and Pharmacy, Iași, Ist Medical Department, Iași, Romania.

Elena Cojocaru - Lecturer, MD, PhD, „Grigore T. Popa” University of Medicine and Pharmacy, Iaşi, Romania.

Răducu Ionuţ Popescu - MD, PhD, Department of Physiology, University of Medicine and Pharmacy „Grigore T. Popa”, Iași, Romania.

Daniela Ababei - Lecteur, MD, PhD, "Gr. T. Popa" University of Medicine and Pharmacy, Faculty of Pharmacy, Pharmacodynamics and Clinical Pharmacy Department, Iași, Romania.

Walther Bild - MD, PhD, Professor Department of Physiology, University of Medicine and Pharmacy „Grigore T. Popa”, Iași, Romania.
\end{abstract}

\begin{abstract}
Throughout life, women make a sequential transition through states modulated by the relative levels of sex steroid hormones that include pre-puberty, menarche, menstruation, pregnancy, breastfeeding and menopause. In addition, exogenous sex hormones can further alter normal hormonal states, so the risk of major depression in multiparous women is twice as high as in nulliparous women and is particularly high during the years after menopause. These "reproductive depressions" involve episodes of depression that occur specifically during the premenstrual, postpartum and perimenopause phases in women. There is substantial evidence that estrogen and progesterone vary considerably throughout a woman's life and contribute to changes in brain structure and function. These findings are consistent with functional data indicating an important role for estrogen and progesterone in mediating emotional processing. The aim of the study was to explore the reciprocal relationships between sex steroid hormones, estrogen and progesterone throughout physiological and pathophysiological conditions in nulliparous females compared to multiparous females and to identify the cause of depression in multiparous females. Material and method: 20 Swiss mice, nulliparous females and $\mathbf{1 5}$ Swiss mice, multipair females with three pregnancies in number,
\end{abstract}


were used, for which the hormonal dosing was done. Single variance analysis (ANOVA) was used. Results: in the group of nulliparous female mice, an increase of the average values of estrogen wad observed, compared to the group of multiparous female mice. Progesterone levels were not significantly different in the two groups of mice. Conclusions: estrogen values changed significantly between the parameters of nulliparous values compared to multiparous female mice, and progesterone values did not change in the two groups of mice studied, suggesting that the increase in estrogen values in multiparous females might cause postpartum depression.

\section{KEYWORDS:}

Postpartum depression, estrogen, progesterone.

\section{INTRODUCTION}

Based on the fact that studies using animal models have provided important perspectives on the pathogenesis, mechanisms and new therapeutic approaches of human diseases, in this study we provide an overview of existing evidence for the physiological, behavioral, cellular and molecular actions of estrogen and progesterone in the context of neurotransmission control in the central nervous system circuits that regulate mood and motivation and discuss the pathology that leads to mental disorders, such as depression in nulliparous and multiparous women.

Postpartum depression is a common complication of childbirth, affecting about $15 \%$ of multiparous women (1). A hormonal aetiology has long been hypothesized due to sudden and substantial fluctuations in steroid hormone concentrations associated with pregnancy and the immediate postpartum period. There is also convincing evidence that estrogen, progesterone and related compounds are important in the activity of the central nervous system at physiological concentrations (2). Estrogen and progesterone are steroid hormones derived from enzymatic changes in cholesterol. In women, the production of estrogen and progesterone takes place primarily in the ovary, but also in the adrenal gland and in other locations, such as adipose tissue. Gonadotropins, follicle- stimulating hormone (FSH) and luteinizing hormone (LH) modulate the synthesis and secretion of ovarian sex hormone. FSH and LH are thus released from the pituitary gland under the control of the central nervous system, mediated predominantly by the hypothalamus, which in turn is widely connected with other areas of the central nervous system $(3,4)$.

Different stages of women's lifespan can be characterized depending on the relative levels of sex steroid hormones. Thus, estrogen and progesterone levels are low throughout childhood, but increase dramatically at the onset of puberty under the influence of pulsatile gonadotropin release from the pituitary gland (5). This process usually leads to the appearance of a regular menstrual cycle, divided into two phases: the follicular phase when serum estrogen levels are high and progesterone low and the luteal phase, in which the progesterone level is high compared to estrogen levels. The late luteal phase is associated with a spectrum of premenstrual symptoms, including headache, bloating, cramps, breast tenderness, weight changes, irritability, decreased concentration, depression and anxiety. The two major biologically active estrogens in non-pregnant women are estrone and estradiol, while pregnant women also produce significant amounts of estriol $(6,7)$ 
During pregnancy, both estrogen and progesterone increase steadily in the three trimesters and then return to baseline after birth. During the postpartum phase, estrogen levels are relatively low, which has been implicated in the pathophysiology of postpartum depression (8). The high level of prolactin present during breast-feeding suppresses the release of gonadotropinreleasing hormone, estrogen and progesterone synthesis and ovulation. (9). Substantial evidence from several sources supports the role of estradiol, the physiological form of estrogen, which is involved in affective regulation, so that estrogen modulates "beneficially" the systems involved in the pathophysiology of depression; regulates the synthesis, metabolism and concentration of classical neurotransmitter receptors involved in depression (serotonin, dopamine and norepinephrine); regulates the activity of the basal and stimulated hypothalamic-pituitaryadrenal axis; acts like antidepressants in stimulating the brain's derived neurotrophic factor; is neuroprotective in a variety of models; improves mitochondrial respiratory efficiency and prevents the formation of oxygen free radicals that are thought to adversely affect the energy of mitochondria in depression; finally, at several levels, it prevents or counteracts the proinflammatory processes described as contributing to depression $(10,11)$.

In this context, the aim of the presentation was to compare the values of hormonal doses for estrogen and progesterone in nulliparous and multiparous female mice, and to identify the etiology of depression in multiparous, starting from the idea that fluctuating levels of the two reproductive hormones trigger a psychiatric affective pathology.

\section{MATERIAL AND METHOD}

Experimental animals used Swiss mice, adult females, of which 20 nulliparous and 15 multiparous weighing 18-20 g, were used for all experiments. The animals were housed in individual cages at a temperature of $21 \pm 2^{\circ} \mathrm{C}$, nictemeral cycle of 12-12 hours., with food and water at their discretion. All animals were kept in similar environmental and feeding conditions.

\section{Blood collection and testing}

In the morning, between 8 and 9 am, Swiss mice were weighed, anesthetized with inhaled isoflurane, and blood samples were collected by taking blood from the left ventricle directly into the vacutainer, approximately $1.5-2 \mathrm{ml} /$ mouse, then placed immediately on ice. The serum was isolated by centrifugation at $2500 x$ g for $15 \mathrm{~min}$. The serum was aliquoted and frozen at $-20^{\circ} \mathrm{C}$ until analysis. Serum estradiol and progesterone were analysed by ELISA (mouse kit Estradiol, cat. RTC009R, BioVendor, Czech Republic; progesterone kit for mice, cat. RTC006R, BioVendor, Czech Republic).

The wells were coated with anti-estradiol antibody and anti-progesterone antibody. An unknown amount of hormone present in the sample competes with a hormone-radish peroxidase (HRP) conjugate for binding to the coated antibody. Each serum sample was analyzed in duplicate with each of the four ELISA kits according to the manufacturer's instructions. The absorbance at each test was read at a wavelength of $450 \mathrm{~nm}$ with a plate reader (State Fax 303 Plus, Awarness Technology Inc, USA). Based on the standard curves on each plate, the hormonal concentration of each hormone was determined for each mouse in the two groups studied. 


\section{Biochemical estimates}

Estrogen dosing in female Swiss mice was performed by the competitive ELISA method, using immobilized antigen. Estradiol is one of the main components of natural estrogens and is the main estrogen secreted during the menstrual cycle. The estradiol calibration range is between $0.312-20 \mathrm{ng} / \mathrm{mL}$, and the limit of detection of estradiol in the body is equal to $0.157 \mathrm{ng} / \mathrm{mL}$.

Progesterone was dosed in female Swiss mice using the sandwich ELISA method, using the HRP-labeled antibody. Progesterone is a female steroid hormone with a variety of physiological effects. In the follicular phase of the menstrual cycle, progesterone is produced at low levels. The progesterone calibration range is between $0.312-20 \mathrm{ng} / \mathrm{mL}$, and the limit of detection of estradiol in the body is equal to $0.173 \mathrm{ng} / \mathrm{mL}$.

\section{Data Analysis.}

We used single variance analysis (ANOVA). Results are expressed as mean \pm SEM. F values for which $\mathrm{P}<0.05$ were considered statistically significant.

\section{RESULTS}

In this study we demonstrated the hypothesis that fluctuations in reproductive hormone levels during pregnancy and the postpartum period trigger postpartum depression in some women, depending on the pathological effects and values of sex steroid hormones such as estrogen and progesterone, using experimental animals - Swiss mice, nulliparous females and multipairs weighing between $18 \mathrm{~g}-25 \mathrm{~g}$. We analyzed two batches of animals, thus, a batch of 20 nulliparous female mice and the second batch of 15 multiparous female mice. For the two groups, a comparison was made between the values of estrogen and progesterone concentrations for each female nulliparous and multiparous mouse.

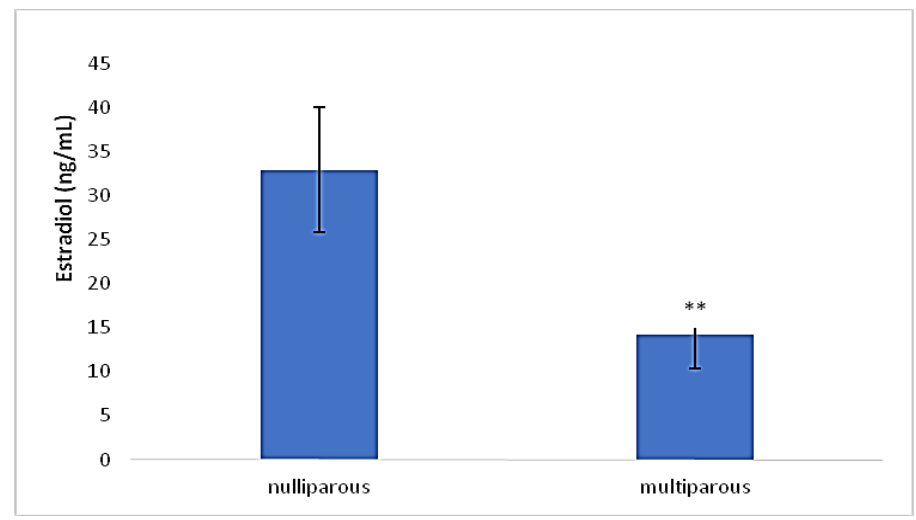

Fig. 1 Mean values in nulliparous and multiparous female mice for the levels of estradiol. The values are mean \pm S.E.M. ( $\mathrm{n}=20$ in nulliparous group and $\mathrm{n}=15$ in multiparous), ${ }^{* *} \mathrm{p}<0.001$ vs. nulliparous group.

Figure 1 represents a difference in the analysis of the average values of the group of nulliparous female mice compared to the multiparous ones, in the analysis of estrogen. Thus, in the group of nulliparous female mice, an increase of the average values is observed, compared to the group of multiparous female mice. 


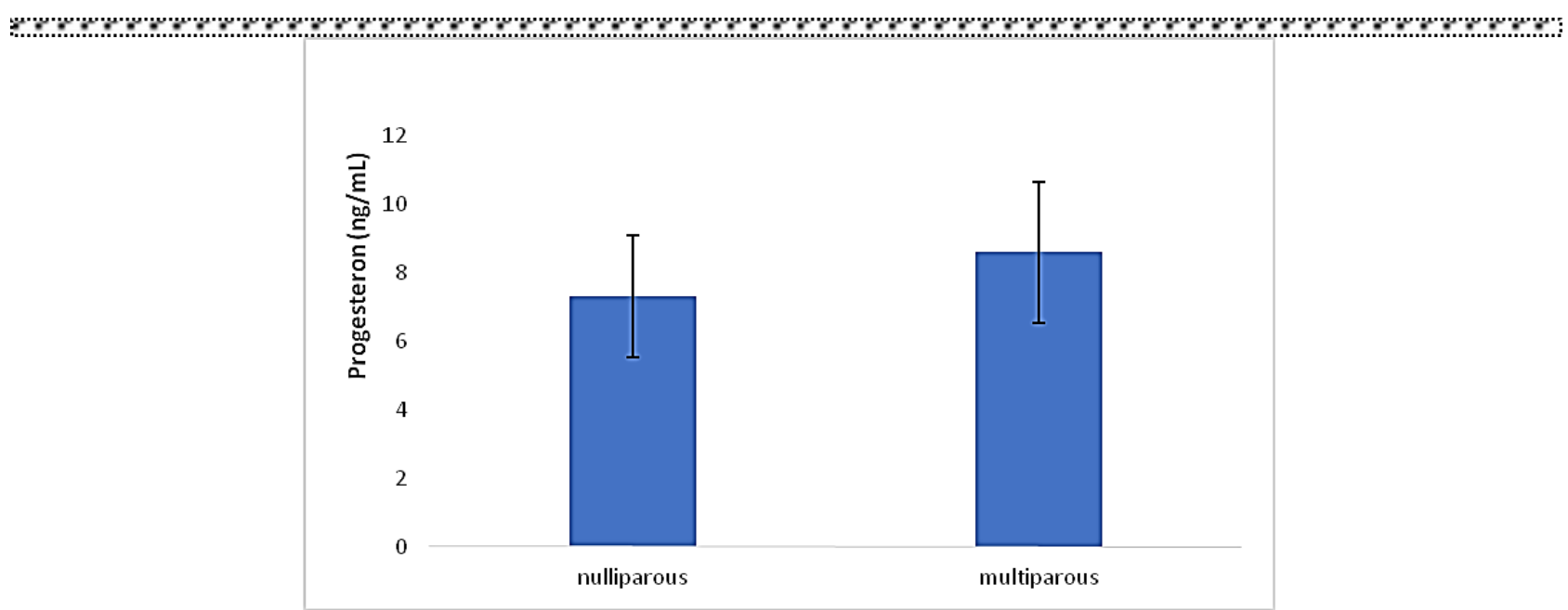

Fig. 2 Mean values in nulliparous and multiparous female mice for the levels of progesteron. The values are mean \pm S.E.M. ( $n=20$ in nulliparous group and $n=15$ in multiparous)

Figure 2 shows no significant difference in the analysis of mean values in the group of nulliparous female mice compared to multiparous female mice in the analysis of progesterone. Thus, progesterone levels are not significant different in the two groups of mice.

Regarding the group differences between the group of nulliparous mice and multiparous mice, we noticed that in multiparous female mice the average estrogen values have a significant increase compared to the nulliparous female, but at the same time, in the progesterone analysis there is no difference in the analysis mean values in the group of nulliparous female mice compared to multiparous female mice. Our results are consistent with the observed epidemiological relationship between the effects of estrogen and progesterone and the type of female mice used, nulliparous and multiparous. However, based on the analysis performed, it was observed that the multiparous female mouse does not suffer pathological changes during the menstrual cycle compared to the nulliparous female mouse, but subsequently may suffer from postpartum depression.

\section{DISCUSSION}

The present study investigated the relationship between the effects of estrogen and progesterone on non-reproductive depression, compared to reproductive depression, using nulliparous and multiparous female mice, in which the two hormones were dosed, thus identifying the hormonal differences found in the two groups of study.

Despite decades of research to identify the causes of postpartum depression (PPD) and to develop effective methods of screening, prevention and treatment, PPD remains common, affecting between 7 and 20\% of women after childbirth. PPD is one of the most important public health problems we can address, it only affects women at an extremely vulnerable time, but it also has harmful effects on children and families. Many have speculated that PPD is caused by the rapid change in the reproductive hormones estradiol and progesterone before and immediately after birth $(12,13)$.

Although a number of studies in human and non-human animals suggest that changes in reproductive hormone levels contribute to PPD (13), several studies have failed to detect 


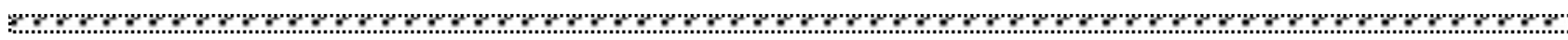

an association between hormone

concentrations and PPD symptoms (14). For example, cross-sectional studies in humans examining differences between groups in ovarian hormone levels and depressive symptoms in the postpartum period have failed to demonstrate the association between absolute concentrations of estrogen, progesterone, and PPD (15). In contrast, studies treating PPD with estradiol have successfully reduced depressive symptoms (16), and animal studies have shown that withdrawal of estradiol and progesterone causes depression-like behavior (17).

Despite all the research that aimed to identify the causes of postpartum depression, PPD remains common and the causes are poorly understood. Many have attributed the appearance of depression to the rapid perinatal change of reproductive hormones. Although a number of studies in human and non-human animals support the role of reproductive hormones in postpartum depression, several studies have failed to detect an association between hormone concentrations and this postpartum depression. There are three main lines of investigation that addressed the role of reproductive hormones in PPD: animal studies, correlated studies of postpartum hormone levels and mood symptoms, and hormone manipulation studies. Reproductive hormones influence virtually any biological system involved in PPD, and a subgroup of women appear to be particularly sensitive to the effects of perinatal changes in hormone levels. The peripartum period is characterized by a rapid and significant physiological change in plasma levels of endocrine hormones, peptides and neuroactive steroids (18). Several lines of research actively examine the potential roles of estrogen, progesterone, cortisol, corticotropin-releasing hormone, adrenocorticotropic hormone, oxytocin, prolactin, testosterone and thyroid function in postpartum depression (19).

Untreated postpartum depression can have substantial adverse effects on the well-being of the mother and child with lasting consequences. PPD with onset during pregnancy is associated with an increased risk of maternal substance abuse, preeclampsia, premature birth and low birth weight. PPD can affect a woman's ability to take care of herself and the baby, having a negative impact on the child's cognitive, behavioral and emotional development (20). Thus, there is evidence that reproductive hormones directly influence the biological systems and neural circuits involved in depression, suggesting that the hormonal instability inherent in the perinatal period could contribute to mood disorder in PPD.

\section{CONCLUSIONS}

This study shows that, depending on the period in which the female was, before or after pregnancy, in the two groups of nulliparous and multiparous female mice, the hormonal concentrations for estrogen and progesterone have very different valuesfrom physiological to pathological. We demonstrated a significant increase between the parameters of estrogen values in the two groups of mice studied, but no significance for progesterone values. Thus, we have a significant increase between the parameters of reproductive hormone fluctuations - estrogen, and for progesterone no significant significance was found between the group of nulliparous and multiparous female mice, which triggers the affective hormonal disorder in some women in the postpartum period. During the postpartum phase, estrogen levels are relatively low, which has been implicated in the pathophysiology of postpartum depression. The study noticed that changes in multiple biological 

systems, such as the immune system, hypothalamic-pituitary-adrenal cortex and lactogenic hormones, contribute to the pathophysiology of postpartum depression.

In conclusion, we suggest that significant changes in estrogen levels in multiparous women can sometimes trigger postpartum depression, and subsequently, can initiate menopause, with detrimental effects on female fertility.

\section{ACKNOWLEDGEMENTS AND DISCLOSURES}

The authors declare that they have no potential conflicts of interest to disclose.

\section{REFERENCES}

1. Gavin NI, Gaynes BN, Lohr KN, Meltzer-Brody S, Gartlehner G, Swinson T. Perinatal depression: a systematic review of prevalence and incidence. Obstet Gynecol. 2005; 106(5 Pt 1):1071-83.

2. Suda S, Segi-Nishida E, Newton SS, Duman RS. A postpartum model in rat: behavioral and gene expression changes induced by ovarian steroid deprivation. Biol Psychiatry. 2008; 64(18471802):311-319.

3. Ahokas A, Kaukoranta J, Wahlbeck K, Aito M. Estrogen deficiency in severe postpartum depression: successful treatment with sublingual physiologic 17beta-estradiol: a preliminary study. J Clin Psychiatry. 2001; 62(5):332-6.

4. Schiller CE, O’Hara MW, Rubinow DR, Johnson AK. Estradiol modulates anhedonia and behavioral despair in rats and negative affect in a subgroup of women at high risk for postpartum depression. Physiol Behav. 2013; 119:137144.

5. Bernstein IH, Rush AJ, Yonkers K, et al. Symptom features of postpartum depression: are they distinct? Depress Anxiety. 2008; 25(1):20-26.

6. Wisner KL, Sit DY, McShea MC, et al. Onset timing, thoughts of self-harm, and diagnoses in postpartum women with screen-positive depression findings. JAMA Psychiatry. 2013; 70(5):490-498.

7. Finocchi C, Ferrari M. Female reproductive steroids and neuronal excitability. Neurol Sci Off J Ital Neurol Soc Ital Soc Clin Neurophysiol. 2011; 32 (Suppl 1):S31-35.

8. Rubinow DR, Girdler SS. Hormones, heart disease, and health: individualized medicine versus throwing the baby out with the bathwater. Depress Anxiety. 2011; 28(4):282-296.

9. Santin AP, Furlanetto TW. Role of Estrogen in Thyroid Function and Growth Regulation. J Thyroid Res. 2011; 2011:e875125.

10. Berent D, Zboralski K, Orzechowska A, Gałecki P. Thyroid hormones association with depression severity and clinical outcome in patients with major depressive disorder. Mol Biol Rep. 2014; 41(4):2419-2425.

11. Krause D, Jobst A, Kirchberg F, et al. Prenatal immunologic predictors of postpartum depressive symptoms: a prospective study for potential diagnostic markers. Eur Arch Psychiatry Clin Neurosci. 2014:1-10.

12. Blackmore ER, Groth SW, (Din) Chen D-G, Gilchrist MA, O’Connor TG, Moynihan JA. Depressive symptoms and proinflammatory cytokines across the perinatal period in African American women. J Psychosom Obstet Gynecol. 2013; 35(1):8-15.

13. Dr. Crystal Edler Schiller, Ph.D., Dr. Samantha Meltzer-Brody, M.D., M.P.H., and Dr. David R. Rubinow, M.D., The Role of Reproductive Hormones in Postpartum Depression, CNS Spectr. 2015; 20(1): 48-59.

14. Tarantino LM, Sullivan PF, Meltzer-Brody S. Using animal models to disentangle the role of genetic, epigenetic, and environmental influences on behavioral outcomes associated with maternal anxiety and depression. Front Psychiatry Front Res Found. 2011; 2:44.

15. Moses-Kolko EL, Fraser D, Wisner KL, et al. Rapid habituation of ventral striatal response to reward receipt in postpartum depression. Biol Psychiatry. 2011; 70(4):395-9.

16. Maguire J, Ferando I, Simonsen C, Mody I. Excitability Changes Related to GABAA Receptor Plasticity during Pregnancy. J Neurosci. 2009; 29(30):9592-9601.

17. Schiller CE, Schmidt PJ, Rubinow DR. Allopregnanolone as a mediator of affective switching in reproductive mood disorders. Psychopharmacology (Berl). 2014:1-11.

18. Djebaili M, Guo Q, Pettus EH, Hoffman SW, Stein DG. The neurosteroids progesterone and allopregnanolone reduce cell death, gliosis, and functional deficits after traumatic brain injury in rats. J Neurotrauma. 2005; 22(1):106118.

19. Michael A, Jenaway A, Paykel ES, Herbert J. Altered salivary dehydroepiandrosterone levels in major depression in adults. Biol Psychiatry. 2000; 48(10):989-995. 
94/Bulletin of Integrative Psychiatry ONew Series O September 2020 O Year XXVI ONo. 3 (86) (1) 20. Eser D, Schüle C, Baghai TC, Romeo E, Rupprecht R. Neuroactive Steroids in Depression and Anxiety Disorders: Clinical Studies. Neuroendocrinology. 2006; 84(4):244-254.

\section{Correspondence:}

Cristina-Maria Gavrilescu,

Lecturer, MD, PhD, „Grigore T. Popa” University of Medicine and Pharmacy, Iași, Ist Medical Department, Iași, Romania. cristina.gavrilescu@ umfiasi.ro

Submission: 28 july 2020

Acceptance: 8 sep. 2020 


\section{BPP Balletin oflategratitive \\ Psychiatry \\ Babetin de Rihiatrie Integrativa}

\section{Case Reports}





\title{
Particularities of a manic episode with psychotic symptoms on the background of psycho-trauma in adolescents
}

\author{
Bianca-Georgiana Secrieru, Elena-Rodica Popescu, \\ Gabriela-Carmen Lupușoru, Roxana Chiriță
}

Bianca-Georgiana Secrieru - M.D., junior psychiatrist, Socola Institute of Psychiatry, Iași Elena-Rodica Popescu - M.D., PhD Student, assistant professor Grigore T. Popa University of Medicine and Pharmacy, Iași; Psychiatrist, Socola Institute of Psychiatry, Iași

Gabriela-Carmen Lupuşoru - M.D., Senior Infantile Neuropsychiatrist, Socola Institute of Psychiatry, Iași

Roxana Chiriță - M.D., PhD, Professor of Psyhchiatry Grigore T. Popa University of Medicine and Pharmacy, Iași, Senior psychiatrist Socola Institute of Psychiatry, Iași

\begin{abstract}
Background: Psychosis is a common and functionally disruptive symptom of many psychiatric, neurodevelopmental, neurologic, and medical conditions and an important target of evaluation and treatment in neurologic and psychiatric practice. Objective: The purpose of this paper is to outline the importance of the evolution of depression, psychosis and mania among adolescents, and to provide a general perspective upon the impact of these disorders on the lives of these young patients and their families. This papers also aims to emphasize the fact that trauma can really affect one's emotional stability, and can be considered a trigger factor for different types of psychiatric conditions. Methods: We present the clinical case of a 17-year old female patient, hospitalized in emergency for psychomotor agitation, aggressive behavior, dromomania, autolytic ideation. Data on the clinical manifestation, trigger factors, treatment and evolution are discussed. Conclusions: When it comes to treating adolescents, whether it is depression, anxiety or other psychiatric disorders, treatment should be prescribed with caution, because the symptomatology is not very clearly defined. This polymorphism can offer a higher level of difficulty when it comes to establishing the right diagnosis.
\end{abstract}

\section{KEY WORDS:}

Depression, psychosis, manic episode, diagnosis criteria, differential diagnosis. 


\section{BACKGROUND}

Psychosis is a common and functionally disruptive symptom of many psychiatric, neurodevelopmental, neurologic, and medical conditions and an important target of evaluation and treatment in neurologic and psychiatric practice (1).

Psychosis is the defining feature of schizophrenia spectrum disorders, a common but variable feature of mood and substance use disorders, and a relatively common feature of many developmental, acquired, and degenerative neurologic and medical conditions. Across these conditions, psychosis is both a contributor to disability and a barrier to productivity and participation. Psychosis is, therefore, an important target of evaluation and treatment among patients receiving care from neurologists and psychiatrists. (2, 3, 4, 5)

Central to all of the presently available guidelines on the evaluation and management of patients with psychosis is the need to identify the cause of psychotic symptoms (in order of priority: delirium, including delirium due to substance intoxication/withdrawal; secondary psychoses of neurologic, medical, and substance use disorders; mood disorders with psychotic features; schizophrenia spectrum disorders; and other psychotic disorders) through comprehensive neuropsychiatric assessment. Patients should be involved in psychiatric, neurologic, and general medical history taking to the extent that their clinical status allows, and, whenever possible, collateral and corroborative history should be obtained from family members or others knowledgeable about the patient and from medical records. Evaluation for potentially causative or contributory medications (e.g., prodopaminergic or anticholinergic agents) and substance use disorders is essential. (6)
Evaluation for manic and depressive episodes (current and lifetime) anchored to DSM-5 criteria should be performed, as strict adherence to these criteria is required to distinguish between schizophrenia mood disorders with psychotic features and schizoaffective disorder. The DMS-5 encourages use of the Clinician-Rated Dimensions of Psychosis Symptom Severity scale to rate the severity of both manic and depressive symptoms associated with schizophrenia spectrum and other psychotic disorders.

Neuropsychological and occupational therapy consultations contribute usefully to cognitive and functional assessments and should be performed when feasible. (7)

The DSM-5 outlines the criteria to make diagnosis of Brief Psychotic Disorder.

Symptoms such as delusions, hallucinations, disorganized speech or catatonic behavior are sufficient. The duration of an episode should be at least 1 day but less than a month, with eventual full return to premorbid level of functioning.

The disturbance is not better explained by major depressive or bipolar disorder with psychotic features such as schizophrenia or catatonia, and is not attributable to the psychological effects of a substance. (e.g., a drug abuse, a medication) or another medical condition. There needs to be specified if the symptoms occur in response to events that singly or together, would be markedly stressful to almost anyone in similar circumstances in the individual's culture (brief reactive psychosis).

Major depression is a chronic illness with a high prevalence and is a major component of 
disease burden. Depressive disorders were the second leading cause of years lived with disability in 2010 in Canada, the United States and globally (Global burden of disease attributable to mental and substance use disorders: findings from the Global Burden of Disease Study 2010). (8)

When depression-related deaths due to suicide and stroke are considered, depression has the third highest global burden of disease. (9)

The DSM-5 outlines the criteria to make diagnosis of depression.

The individual must be experiencing five or more symptoms during the same 2-week period and represent a change from previous functioning; at least one of the symptoms is be either (1) depressed mood, or (2) loss of interest or pleasure.

Symptoms include: depressed mood most of the day, nearly every day, as indicated by either subjective report, or observation made by others. Markedly diminished interest or pleasure in all, or almost all activities most of the day, nearly every day. Significant weight loss, or decrease or increase in appetite nearly every day. Insomnia or hypersomnia nearly every day. Psychomotor agitation or retardation nearly every day (observable by others, not merely subjective feelings of restlessness or being slowed down). Fatigue or loss of energy nearly every day. Feelings of worthlessness or excessive or inappropriate guilt nearly every day. Diminished ability to think or concentrate or indecisiveness nearly every day. Recurrent thoughts of death, recurrent suicidal ideation without a specific plan or a suicide attempt.

These symptoms must cause clinically significant distress or impairment in social, occupational, or other important areas of functioning.
The episode must not be attributed to the psychological effects of a substance or to another medical condition.

Mania, or a manic phase, is a period of 1 week or more in which a person experiences a change in normal behavior that drastically affects their functioning. Mania is different from hypomania because hypomania does not cause a major deficit in social or occupational functioning, and it is a period of at least 4 days rather than 1 week. The defining characteristics of mania are increased talkativeness, rapid speech, decreased the need for sleep (unlike depression or anxiety in which the need for sleep exists, but there is an inability to sleep), racing thoughts, distractibility and psychomotor agitation. Some other hallmarks of mania are an elevated or expansive mood, mood lability, impulsivity, irritability, and grandiosity. If the individual experiencing these symptoms requires hospitalization, then this period automatically qualifies as true mania and not hypomania, even if the symptoms are present for less than one week. (10-11).

Many families bring their loved ones to the emergency room due to the excessive behavioral changes they have noticed over a brief period. Patients amid a manic phase commonly engage in goal-directed activities that may result in harmful consequences, such as spending excessive money, starting businesses unprepared, traveling, or promiscuity. Many patients engage in property damage or even harm themselves or others through verbal or physical assaults. They may also become highly aggressive, agitated, or irritable. Although the patient may have poor insight and may not recognize they are behaving out of the norm, it becomes apparent to family or friends that this behavior may be due to mental illness. (12) 


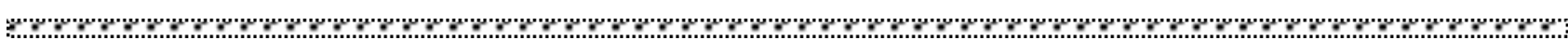

The DSM-5 outlines the criteria to make diagnosis of a Manic Episode

The patient presents an elevated, expansive or irritable mood and abnormally and persistently increased goal-directed activity or energy, lasting at least one week and present most of the day, nearly every day. During the period of mood disturbance and increased energy or activity, three or more of the following symptoms are present to a significant degree and represent a noticeable change from usual behavior: inflated selfesteem or grandiosity, decreased need for sleep, more talkative than usual or pressure to keep talking, flights of ideal or subjective experience that thoughts are racing, distractibility, excessive involvement in activities that have a high potential for painful consequences.

The mood disturbance is sufficiently severe to cause marked impairment in social or occupational functioning or to necessitate hospitalization to prevent harm to self or others, or there are psychotic features.

The episode is not attributable to the physiological effects of a substance (a drug abuse, a medication, other treatment) or to another medical condition.

A full manic episode that emerges during antidepressants treatment (e.g., medication, electroconvulsive therapy) but persists at a fully syndromal level beyond the physiological effect of that treatment is sufficient evidence for a manic episode and, therefore, a bipolar I diagnosis.

\section{CASE PRESENTATION}

Reasons for admission at the hospital:

A 17-year old female patient from Suceava presents for admission to the "Socola" Institute of Psychiatry, Iași, accompanied by her parents for sudden, onset of psychomotor restlessness, aggressive behavior, dromomania, autolytic ideation, emotional lability. False beliefs, inappropriate behavior, difficulties carrying out daily activities were also observed.

\section{PAST PERSONAL MEDICAL HISTORY AND FAMILY HISTORY}

The parents declare their daughter was diagnosed with depression a year prior following a traumatic event.

Family history- noncontributory.

\section{HISTORY OF PRESENT ILLNESS}

The family reports that a year prior, the patient was diagnosed with severe depression. The episode followed what the patient named "a traumatic event". The sister got married and moved to another country; the poor relationship with the brother in-law and the sister's departure have had a huge impact on the teenager. She was treated as an outpatient, the treatment consisting in antidepressants. She has received this treatment for a year.

The relationship with the parents is poor and the patient is accusing them of abuse of authority. She resents her father and she affirms that the relationship with her mother is superficial. She cares the most about her sister, and now she believes that she abandoned her. The autolytic ideation appeared following a set of events.

Parents affirm that during the month prior the hospitalization, the patient showed an aggressive behavior towards them, mostly verbal, lost interest in all kinds of activities, poor activity in school, disorganized behavior, weight loss, emotional lability. The patient refused psychological counselling. 
A month prior hospitalization, the patient has been involved in a car accident. No severe trauma has been detected. The patient affirms that the injuries she suffered were severe and had a huge impact on her well-being, affecting her mental health the most. The driver was her boyfriend at the time. After the accident, the boyfriend left the country for work. The patient refers to this episode as highly traumatizing and once again, she feels abandoned.

A few weeks after the accident, the patient visited a male friend. The patient affirms that this friend has abused her sexually, being held hostage. She affirms that the sexual act was not consented and that she was a rape victim. She did not call the police or her parents.

She was later on taken to the Emergency Room where no signs of violence have been detected. She kept affirming that she was raped, therefore a gynecological examination was required in order to establish if she was a victim of a sexual assault; no signs of sexual abuse were detected. A pregnancy test was also made. The result was negative.

They redirect the patient to our clinic and the next day she is admitted.

\section{THE PSYSICAL EXAM}

Did not lead to any pathological findings.

The Clinical Examination reveals a nervous female patient, a BP of 109/69 mmHg, HR of 71, rhythmic heart sounds, normal pulmonary auscultation, no abdominal pain on palpation.

An ECG was performed and it showed sinus rhythm and no signs of ischemia.

\section{THE PSYCHIATRIC EXAM}

\section{Appearance and General Behavior:}

Attitude: cooperative, aggressive behavior, curious, psychomotor restlessness, emotional lability.
Clothing: neat, good hygiene status.

Voice: accelerated verbal flow, emotionally modulated, voice of medium tonality and increased intensity.

Look: establishes and maintains visual contact with the examining physician.

Facies: mobile

Mimics and Pantomime: gestural activity slightly increased.

\section{Cognitive functions:}

Sensation: slight hyperaesthesia.

Perception: she denies the presence of hallucinatory phenomena.

Attention: spontaneous hyperprosexia, marked distraction.

Memory: selective evocation hypermnesia for events related to the patient's personal life.

Thinking unfolds in a disorganized, accelerated rhythm, presenting pressured speech, racing thoughts, delusions of grandiosity.

Imagination: without disturbances.

\section{Affective and motivational functions:}

Mood: elevated

Agressive behavior and negative emotions towards the family.

Feelings: inadequate.

Passions: insufficiently structured.

Motivation: periods of delay in initiating activities.

Instincts

- $\quad$ eating-diminished

- preservation- autolytic ideation

- sexual- exacerbated.

\section{Executive functions:}

Volition: high spontaneity but with low involvement in activites, excessive in goaldirected activities (she wants to leave the country and get two jobs; she also wants to finish her studies with the highest grades and study medicine). 
ryor

Motor activity: self conduct and self care abilities maintained.

Verbal activity: rapid, difficult to interrupt, pressured speech

Behavior: uninhibited, theatrical behavior which included dramatic mannerisms.

Sleep: decreased need for sleep.

\section{Judgement and Insight:}

Conscience: orientation in space, time, and person preserved.

Insight over illness: absent.

Intellect: in accordance with educational background.

Character: inappropriate attitude.

Psychological examination: manic configuration with elevated mood, accelerated thought process, instability traitsm integrative and relational deficiency.

She presents a theatrical attitude, demonstrative behavior, affirms that she was the victim of a sexual trauma (rape), emotional lability, low tolerance to minor frustration, irritability, irascibility.

POSITIVE DIAGNOSIS: Manic episode with psychotic symptoms.

\section{DIFFERENTIAL DIAGNOSIS}

1. Organic affective disorders (organic manic disorder); a month prior admission, a CT scan was performed. No abnormalities were observed. Following her admission, we eliminated somatic diseases and the consumption of drugs.

2. Psychotic disorders, primarly delusional- the symptoms of this disorder have an abrupt onset, with no prodromal phase, usually caused by a traumatic event and the symptoms are are connected thematically with the stressful situation; the patient's delusions are mainly of grandeur.

3. Psychotic disorders induced by certain life conditions or by substance abuseno substance abuse was detected.

4. Schizoytypal disorder: symptoms which meet the criteria for this disorder include ideas of reference, odd beliefs with mystical or paranormal influences, observations that could not be made in this patient's case.

5. Persistent delusional disorders: include a multitude of pathologies that are characterized by the presence of delusions for at least 3 months.

6. Antisocial personality disorder: this disorder is characterized by the lack of respect towards other people and other people's rights. The patient in incapable to adapt to social and legal norms.

7. Schizoaffective disorder: both affective and schizophrenic symptoms can be observed during the same episode. No schizophrenic symptoms can be observed in this case.

During hospitalization the patient was under strictly supervised treatment.

The patient was anxious, showed signs of irritability, claiming, "you are going to have to deal with me from now on!", theatrical behavior.

The following days, the patient's evolution was stationary. She maintained the aggressive behavior. She had elevated self-esteem, and kept threatening the medical staff "I am going to unleash hell on all of you", "Enough with being a good girl. I am going to become a devil", "I am not crazy, I am just acting crazy", "I declare war", "You are going to see what I am capable of".

The patient accused the doctor and her parents of abuse of power "I want you to know that 


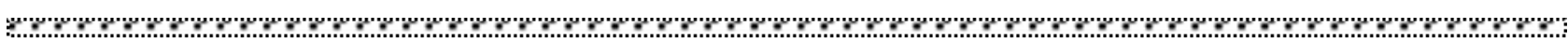

you have disappointed me, yes, you, the ones who were supposed to take care of me; you abandoned me in a mental institution! Why? Why am I always wrong? Set me free! It is only your fault! Lack of love!".

She insisted on the fact that she was pregnant following the "rape".

Another pregnancy-test was made. The result was negative. She then refused a gynecological examination.

After a few days of treatment, the treatment consisting in antipsychotics, the evolution was still stationary. The patient became demanding, she still presented aggressive behavior. "I am not crazy! I am just pregnant".

"Her mother's hope...cleaning tables dreaming about the day when she will wear a white robe and she will be walking down the halls of the hospital, bringing comfort to the patients". "May God be with you. Don't forget to talk to Him!".

A few days after continuing the treatment, the patient began to follow the rules. She insisted on helping the medical staff with all the chores and activities. She praised the doctor and the nurses. She told everyone she loved them. Her behavior suffered obvious adjustments. She was calm, cooperative, communicative, she showed no signs of aggressive behavior. "My family is perfect for me. Mum + Dad= Me + My sister", "I absolutely adore and love these people", "My dear parents, my love for you is infinite", "My family is loving, perfect", "I thank God every single day for my family", "My dear parents, please, I want you to understand! I want to come home! Please take me home! I am going to be how you want me to be; just take me home".

\section{DISCUSSIONS}

This case is illustrative for the evolution of psychiatric disorders in adolescents. After the first traumatic event, the patient was diagnosed with depression. She received treatment which consisted in antidepressants for a year. The symptomatology at the moment of admission indicated a psychotic episode, taking into consideration her false beliefs, inappropriate behavior and difficulties carrying out daily activities. The severity of this case is represented by the autolytic ideation and dromomania, the refusal of counselling or any type of social support. On her admission, antipsychotic was prescribed. Some of the symptoms disappeared, but, unfortunately, a shifting from psychosis to mania has been observed within a few days.

The patient had a decreased need for sleep, poor judgement, grandiose beliefs, disconnected thoughts, inappropriate irritability, pressure to keep writing to her parents, attention was easily drawn to unimportant and irrelevant items. Her speech was always rapid, difficult to interrupt, she presented a theatrical behavior which included dramatic mannerisms. Taking into consideration the relationship with her family, her speech was always marked by hostile comments and complaints. Flight of ideas was also observed.

The strong religious beliefs, the excessive authority found within the family environment, the supposedly abandonment involving her sister, the car accident, the departure of her boyfriend and the misunderstanding that she has come across for the past few months, severely affected the patient's emotional stability. She refers to these events as highly traumatizing. She resents her family, she believes that her situation was caused by the people who were supposed to take care of her and to offer support. No evidence of self-guilt was observed. 


\section{CONCLUSIONS}

We need to underline the importance of trauma, especially when it comes to adolescents. Trauma can really affect one's emotional stability and evolution. In this case, the patient's family was dysfunctional, she felt emotionally abused, she felt abandoned and misunderstood. The car accident can also be included on the list of stressors. Even if no evidence of a sexual assault exists, the patient is firmly convinced that she is a rape victim. This last event also influences the evolution, being considered by the patient as highly traumatizing.

When it comes to treating depression in adolescent patients, caution is required. Our case report suggests that the prescription of antidepressants to adolescents can be followed by severe complications, such as the shift to psychosis.

Emotional support is essential when it comes to treating depression. A safe and warm environment must be provided in order for the patient to feel comfort and understanding from the family members and society. Therefore, psychological counselling is highly recommended.

\section{ACKNOWLEDGEMENTS AND DISCLOSURES}

The authors declare that they have no potential conflicts of interest to disclosure.

\section{REFERENCES}

1. David B. Arciniegas, Psychosis, Continuum (Minneap Minn) 2015 Jun; 21 (3 Behavioral Neurology and Neuropsychiatry).

2. Morgan C, Lappin J, Heslin M, et al. Reappraising the long-term course and outcome of psychotic disorders: the AESOP-10 study. Psychol Med 2014; 44 (13): 2713-2726. doi:10.1017/S0033291714000282.

3. Rabinowitz J, Berardo CG, Bugarski-Kirola D, Marder S. Association of prominent positive and prominent negative symptoms and functional health, well-being, healthcare-related quality of life and family burden: a CATIE analysis. Schizophr Res 2013; 150 (2-3): 339-342. doi:10.1016/j.schres.2013.07.014.

4. Vilalta-Franch J, Lopez-Pousa S, Calvo-Perxas L, Garre-Olmo J. Psychosis of Alzheimer disease: prevalence, incidence, persistence, risk factors, and mortality. Am J Geriatr Psychiatry 2013; 21 (11): 1135- 1143. doi:10.1016/j.jagp.2013.01.051.

5. Forsaa EB, Larsen JP, Wentzel-Larsen T, et al. A 12-year population-based study of psychosis in Parkinson disease. Arch Neurol 2010; 67 (8): 996- 1001. doi:10.1001/archneurol.2010.166. Lehman AF, Lieberman JA, Dixon LB, et al. Practice guideline for the treatment of patients with schizophrenia, 2nd ed Arlington: American Psychiatric Association, 2004.

6. Clinician-rated dimensions of psychosis symptom severity. American Psychiatric Association. Published 2013. Accessed April 9, 2015.

7. Whiteford HA, Degenhardt L, Rehm J, Baxter AJ, Ferrari AJ, Erskine HE, Charlson FJ, Norman RE, Flaxman AD, Johns N, Burstein R, Murray CJ, Vos T

Lancet. 2013 Nov 9; 382(9904):1575-86.).

8. Ferrari AJ, Norman RE, Freedman G, et al. The burden attributable to mental and substance use disorders as risk factors for suicide: findings from the Global Burden of Disease Study 2010. PLoS ONE. 2014;9;e91936.

9. Wang YY, Xu DD, Feng Y, Chow IHI, Ng CH, Ungvari GS, Wang G, Xiang YT. Short versions of the 32-item Hypomania Checklist: A systematic review. Perspect Psychiatr Care. 2020 Jan;56(1):102-111. . Tazawa Y, Wada M, Mitsukura Y, Takamiya A, Kitazawa M, Yoshimura M, Mimura M, Kishimoto T. Actigraphy for evaluation of mood disorders: A systematic review and meta-analysis. J Affect Disord. 2019 Jun 15;253:257-269.

10. Kavanagh BE, Brennan-Olsen SL, Turner A, Dean OM, Berk M, Ashton MM, Koivumaa-Honkanen H, Williams LJ. Role of personality disorder in randomised controlled trials of pharmacological interventions for adults with mood disorders: a protocol for a systematic review and meta-analysis. BMJ Open. 2019 May 01;9(4):e025145.

\section{Correspondence:}

Elena-Rodica Popescu-M.D.

PhD Student, psychiatrist, "Socola” Institue of Psychiatry, Iași, România, no. 36 Str. Bucium, elenapopescu.88@yahoo.com 


\section{BP \\ Psychiatry \\ Buletin de Psilhiatrie Intejurativă}

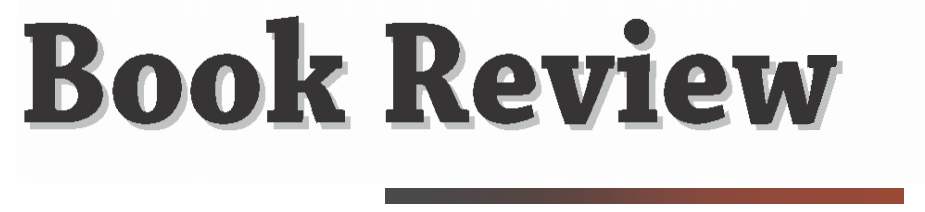





\title{
The Center Cannot Hold. The story of my madness
}

\author{
The anti stigma manifesto of Elyn Saks \\ Review signed by Ilinca Untu, Roxana Chiriță
}

Ilinca Untu - M.D., Ph.D. Student, "Socola” Institute of Psychiatry, Iaşi, Romania, Grigore T. Popa University of Medicine and Pharmacy Iași

Roxana Chiriță - Professor, M.D., Ph.D., "Socola" Institute of Psychiatry, Iaşi, Romania, Grigore T. Popa University of Medicine and Pharmacy Iași

The Center Cannot Hold. The story of my madness, autobiographical book published by Elyn R. Saks in 2007 is translated into Romanian for Trei Publishing House in 2016, by Laura Netea. This is an honest testimony of the author's personal story and professional career, early diagnosed with paranoid schizophrenia, reflecting its successes and failures in close connection with the evolution of mental illness, the underlying message being optimistic, nullifying clichés rooted in the collective mind, the book being a true anti stigma manifesto.

Elyn R. Saks is an Associate Dean and Professor of Law, Psychology, and Psychiatry and the Behavioral Sciences at the University of Southern California Gould Law School, an expert in mental health legislation and Mac Arthur Foundation Fellowship winner. She graduated from Oxford as a Marshall Scholar and received her J.D. from Yale Law School. She has published three books and several dozen articles and is part of the management board of several mental health foundations. She currently lives in Los Angeles with her husband, Will Vinet.

Saks lives with the diagnosis of schizophrenia and wrote about her experience with this major mental disorder in her award-winning best-selling autobiography, The Center Cannot Hold, published by Hyperion Books in 2007. As an irony of fate, the author is also a survivor of cancer, a nosological category often considered to be associated extremely rarely with schizophrenic spectrum disorders.

Saks graduated summa cum laude from Vanderbilt University before earning her master of letters from Oxford University as a Marshall Scholar and her Jurist Doctor Degree from Yale Law School, where she also edited the Yale Law Journal. The author holds a $\mathrm{PhD}$ in psychoanalytic sciences from the New Center for Psychoanalysis. Saks is a 


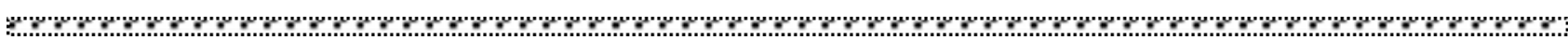

member of Phi Beta Kappa, the oldest academic honor society in the United States, and often described as the most prestigious one, an affiliate member of the American Psychoanalytic Association, a board member of Mental Health Advocacy Services and a member of the Los Angeles Psychoanalytic Foundation, Robert J. Stoller Foundation, and American Law Institute, receiving numerous awards for her professional and research work.

Saks experienced the first symptoms of the disease at the age of eight, which were somewhat overlooked by her relatives. Yale Law School experience was a crucial moment for the author that corresponded to the turning point from which she was forced to follow the treatment with antipsychotic medication. In this existential context, Saks argues that no disease should define anyone, thus referring to the social and individual tendencies to stigmatize the sick and especially those suffering from a mental disorder.

The author militates for the demolition of the myths regarding the concept of mental illness in everything that means its manifestations and impact, by promoting people with such diagnoses who do not lead a life full of pain and deprivation, but on the contrary, performs personally and professionally absolutely admirable things, even if with more effort,. The successful prognosis of mental disorders includes medication, often psychotherapy, socalled luck and especially the individual inner strength to fight their own demons. This power has several sources of origin such as love, forgiveness, faith, interpersonal relationships. The author's testimony is that "we who struggle with these disorders can lead full, happy, productive lives, if we have the right resources."

On numerous occasions, Elyn Saks has lectured at the American Psychiatric
Association about her perspective on what psychiatric care should be like. The extraordinary success of becoming a successful professional despite her diagnosis, perceived in the collective mind as extremely disabling. The author's research is based on the particularities of highly functional people who face a diagnosis of schizophrenia.

The Center Cannot Hold (Romanian edition) is 436 pages long and is structured in twentyfour chapters in which the author speaks with surprising honesty about childhood, the first signs of illness, adolescence, basic family relationships, studies, the first diagnosed psychotic episodes, but in addition she approaches with great depth the problematic of adherence and the establishment of a therapeutic highlight through the prism of one's own experience, of personal obstacles in accepting psychotropic medication, of the psychotherapeutic path of psychoanalytic invoice. At the same time, the author presents her professional and relational course, making a conclusive parallel with the course of her illness. The overall message is a positive one, full of acceptance and struggle, of understanding one's own situation, of adapting and retrieving. As a general idea, Elyn Saks' book is like a detailed case study that tells the story of a person diagnosed with schizophrenia in the 70s who has had a meritorious evolution so far, but sprinkled with effort and difficulties and which overlaps the history of modern psychiatry by changing paradigms, antipsychotics, psychosocial approaches. It is also interesting to fallow Saks' experience as an inpatient, the interaction with other patients and then the readjustment to a normal life, the meeting of the soul mate and remaining in a love relationship until now.

This book is unique in that it breaks down many patterns and especially demystifies what is generally believed to be schizophrenia 
and schizophrenic spectrum disorders. The author diagnosed with schizophrenia becomes a successful professional and advocates for the rights of people with mental illness, without idealizing, exposing in a realistic and honest way symptoms, deficits, pathological elements remaining despite treatment, relapses, finds the ideal recipe to show how nothing is impossible. The Center Cannot Hold can be a source of inspiration for anyone suffering or not from a mental illness, but especially an indisputable proof that a psychiatric disorder should not and may not be a condemnation to a life of torment and giving up. The perspective that Elyn Saks offers us is a rare but plausible one, and her story is a living testimony of the fact that everything is possible.

The volume concludes with a chapter of thanks addressed to all the representative figures in the author's life and with one on its current status, talking especially about this book and how it was received by the public.

Finally, the Romanian edition of The Center Cannot Hold presents an interview with Elyn Saks, in which she talks about her relationship with her husband, what it means for her to be psychotic and how her functioning and career are influenced by psychotic episodes. Somehow, she emphasizes the importance of the fact that in mental health classes she speaks from a personal experience, so the information is transmitted more authentically and more credibly.

One of the most important messages of the author addressed to the readers is crystallized in: I hope that, giving the mental illness a face, I can remove at least a little from the stigma. 



\section{Instructions for authors}

The Bulletin of Integrative Psychiatry publishes blind peer reviewed articles, reviews, updates, meta analyses, original papers, case reports in the following fields of research: psychiatry correlated with philosophy, ethics, meta ethics, bioethics, aesthetics, economy, sports, nutrition, law, forensic medicine, political sciences, communication science ,sociology, social assistance, anthropology, cultural anthropology, literature, religion. The journal is published quarterly in English.

Articles are submitted to the discussions in meetings of the Editorial Board, being subjected to a blind peer review evaluation from two specialists in the field.

Evaluating the scientific value and originality of the article, as well as its layout, grammar and structure and the way in which the guidelines for publication have been observed, the final publication decision is made by the editor-in- chief and the advisory board. It is also required that the article admitted for publication should represent a substantial contribution in the field, and the data included are correct and pertinent.

There are no author fees and all articles are made free access on line.

In the Declaration of Interests, authors must indicate any financial and personal relationships with other people or organizations that could influence in an incorrect way their work.

Conflict of interests can refer to financial relationships and academic competition.

Authors should not submit articles that have been published or sent for publication to other journals.

Bulletin of Integrative Psychiatry, accept for publication the following types of works (articles): reviews, updates, meta analysis, original papers, case report

Fields of research: psychiatry correlated with philosophy, ethics, metaethics, bioethics, esthetics, economy, sports, nutrition, law, forensic medicine, political sciences, communicational sciences, sociology, social assistance, anthropology, cultural anthropology, literature, religion etc.

\section{Manuscript Criteria and Information}

Manuscripts and all attached files should be submitted in electronic form and on paper.

The electronic form should be submitted, either on compact disk or by e-mail (see at end). It is preferable that two copies (in English and Romanian) of the manuscript, printed on one side of A4 paper format, single-spaced, with $2 \mathrm{~cm}$ margins, be also submitted to the same address. The manuscript should be accompanied by a cover letter including the statement on authorship responsibilities form, the statement on ethical considerations and the statement on financial disclosure (For more information please see directions on website).

\section{Authorship}

All named authors should meet the criteria for authorship as stated in the "Uniform Requirements for Manuscripts Submitted to Biomedical Journals: Writing and Editing for Biomedical Publication" issued by the International Committee of Medical Journal Editors (www.icmje.org). 


\section{Ethical considerations}

If the scientific project involves human subjects or experimental animals, authors must state in the manuscript that the protocol has been approved by the Ethics Committee of the institution within which the research work was undertaken. All authors are responsible for adhering to guidelines on good publication practice.

\section{Financial Disclosure}

The manuscript should be accompanied by a cover letter including the statement on financial disclosure. The statement on conflicts of interest will be published at the end of the paper. Please submit all requested signed documents by regular mail to the Secretariat. Scanned copies sent electronically and fax submissions are not acceptable.

\section{Corrections}

Scientific fraud is rare events; however, they have a very serious impact on the integrity of the scientific community. If the Editorial Board uncovers possible evidence of such problems it will reserves the right to take all steps it considers necessary for the elucidation of the situation, including possibility to publish errata, corrigenda, or retractions.

\section{Copyright}

The papers published in the Journal and protected by copyright. Their full or partial publication in other journal is allowed only with the written approval of the publisher.

\section{Manuscript Preparation}

- Romanian authors should send both the Romanian and English version of the article, including title, abstract and key words. Foreign authors should send the English version of the article.

- Manuscripts must be prepared in conformity to the "Uniform Requirements for Manuscripts Submitted to Biomedical Journals: Writing and Editing for Biomedical Publication" issued by the International Committee of Medical Journal Editors (www.icmje.org).

- Articles must be written in Microsoft Word, Style: Normal + Justify, Font: Times New Roman, size 12. All manuscripts must be typed single-spaced. Original source files (not PDF files), are required. In text editing, do not use spacing with spacebar, or paragraph mark, use only Enter or Tab key.

- Subheadings of the article should be left-justified, typed with capital letters, Font: Times New Roman, size 12.

- The abstracts and Key words must be written in Microsoft Word, Style: Normal + Justify, Font: Times New Roman, size 12.

- Use as few formatting commands as possible:

o input your text continuously (without breaks);

o do not use different types of fonts to highlight your text;

$\circ$ any word or phrase that you would like to emphasize should be indicated throughout the text by underlining;

○ use only the "Enter" key to indicate the end of the end of paragraphs, headings, lists etc.;

o do not use the "Space Bar" to indicate paragraphs, but only the "Tab" key

- Figures must be cited in order in the text using Arabic numerals, (e.g., fig.2). Their width should be max. $13,5 \mathrm{~cm}$. The figures have to satisfy the following conditions: 
- Black and white photographs with good contrast, with recommended size;

- Scanned photograph with a resolution of minimum 300 dpi and edited on a computer, original file (*JPG);

- Illustrations (drawings, charts) created on a computer, cited in the text, original file (*XLS, *CDR). Every figure should be accompanied by a title and a legend.

- Tables, numbered consecutively with Latin numerals (e.g., Tab. II), should have a width of max. 13,5 cm. Every table should be also accompanied by a title and a legend. The distribution of tables and figures in the text should be balanced.

- Please do not import tables or figures into the text document, but only specify their insertion in text (e.g., Table No.III insertion). They have to be sent in separate files. Files should be labeled with appropriate and descriptive file names.

- Abbreviations shall be avoid. If used, will occur preceded by the full term at their first apparition in text. A list of all used abbreviations shall be made at the end of the article,

- Separate pages: tables, graphics, pictures and schemes will appear on separate pages.

- You may use a common compression program: ARJ, RAR or ZIP.

\section{Manuscript organization}

Article title: titles should be short, specific, and descriptive, emphasizing the main point of the article. Avoid a 2-part title, if at all possible. Do not make a declarative statement in the title. Title length, including punctuation and spaces, ideally should be under 100 characters and must not exceed 150 characters.

\section{Manuscript Size}

- Original paper - 8 pages/3500 words;

- Review and update - 8 pages/3500 words;

- Case report - 4 pages/1700 words;

- Clinical notes - 3 pages/1300 words;

- Letters to editor, information scientific reunions - one page/450 words.

Author(s). First name, middle initials and surname of the authors, without any scientific, didactic or military degrees; (e.g., Mircea A Popescut, Aura Vasilescu, not Popescu M.A., Vasilescu A.).

Footnote that specifies the authors' scientific titles, professional title, name and address of their workplaces (institution and department) for each author; contact details of the corresponding author (full address, telephone number, fax number, e-mail address) and the address of the institution and department where the study has been carried out. Contact details will be published unless otherwise requested by the author.

Abstracts in in English and Romanian, should have max. 300 words. For original articles is advisable to include five paragraphs, labeled Background, Objective(s), Method(s), Result(s), and Conclusion(s).

Keywords maximum of 6 keywords (minimum of 3). Keywords should not repeat the title of the manuscript. 


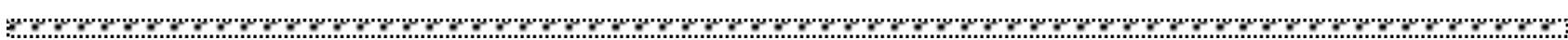

Original papers organized in:

- Introduction (no more than $25 \%$ of the text), material and methods, results, comments or discussions and acknowledgements.

- Material and methods have to be described in enough detail to permit reproduction by other teams. The same product names should be used throughout the text (with the brand name in parenthesis at the first use).

- Results should be presented concisely. Tables and figures should not duplicate text.

- Discussions should set the results in context and set forth the major conclusions of the authors.

- Information from the Introduction or Results should not be repeated unless necessary for clarity. The discussion should also include a comparison among the obtained results and other studies from the literature, with explanations or hypothesis on the observed differences, comments on the importance of the study and the actual status of the investigated subject, unsolved problems, questions to be answered in the future.

- Acknowledgements section must disclose any substantive conflicts of interest in addition to the customary recognition of non-authors who have been helpful to the work described.

References. References should be numbered consecutively in the order in which they are first mentioned in the text. Identify references in text, tables, and legends by Arabic numerals in parentheses. The reference list will include only the references cited in the text (identified by Arabic numerals in parentheses, not in square brackets and not bold). All authors should be listed when four or less; when five or more, list only the first three and add "et al" (Popescu I, Popescu I, Vasile V. et al). The name of the Journals cited in the References should be abbreviated according to ISI Journal Title Abbreviations.

\section{It is absolutely imperative that references to be entered in the text!!}

\section{Examples:}

- Reference to a journal publication:

Amura SG.: Neurotransmitter transportor: recent progress. Ann Rev Neurosci 1993; 16; 73-93.

Tonis PJ, von Sandick JW, Nieweg OE, et al.: The hidden sentinel node,in breast cancer. Eur J Nucl Med 2002; 29: 305-311

\section{- Reference to a book:}

Ashcroft FM.: Ion channels and disease, San Diego, London: Academic Press, 2000, pp. 26-52.

\section{- Reference to a chapter in an edited book:}

Pandiom MR, Fischer DA. Immunoradiometric assays. In: Scabes CG, editor. Handbook of Endocrine Research Techniques. New York, London: Academic Press, 1993, 26-52 The punctuation and the general aspect of the text format must comply with the rules mentioned above. This is a mandatory and eliminatory condition.

\section{ACKNOWLEDGMENTS AND DISCLOSURE}

Authors: an "author" is generally considered to be someone who has made substantive intellectual contributions to a published study, and biomedical authorship continues to have important academic, social, and financial implications (1). An author must take responsibility for at least one 

component of the work, should be able to identify who is responsible for each other component, and should ideally be confident in their co-authors' ability and integrity.

We strongly encourage developing and implementing a contributor ship policy, as well as a policy on identifying who is responsible for the integrity of the work as a whole.

Disclosure: The authors must declare if they have or not a potential conflicts of interest to disclose. Acknowledgments: All contributors who do not meet the criteria for authorship should be listed in an acknowledgments section. Authors should declare whether they had assistance with study design, data collection, data analysis, or manuscript preparation. If such assistance was available, the authors should disclose the identity of the individuals who provided this assistance and the entity that supported it in the published article. Financial and material support should also be acknowledged.

\section{PEER REVIEW PROCESS}

Although the practice of peer review started 300 years ago, only since 198s the process has been active to analyze if articles sent to journals are well-written, correctly edited and represent reliable sources of information.

All manuscripts intended for publication will be subject to blind peer-review by a committee of experts which assesses the scientific and statistical correctness of articles submitted. The committee receives the manuscripts without knowing the authors' name and proposes possible changes, which will be transmitted to the authors by the medium of Editorial Board. The authors have the obligation to oversee the text in English language with the help of a professional translator.

When submitting a paper, a confirmation email is automatically sent back to the author. It contains a unique registration number used as a referral in further correspondence.

Initially, the editorial team verifies whether the manuscript complies with the editing instructions. If the paper does not meet the necessary requirements, it is rejected and the corresponding author is notified to correct the errors. If all instructions for editing have been followed accordingly, the editor selects two reviewers that will independently evaluate the paper. They have high expertise in the "peer-review" system and are well-known specialists in the field. The reviewers have all academic affiliations and may be already accredited by the Journal or specifically contacted for certain papers. Their affiliation is usually different from the one of submitting authors.

The editor sends the paper in both electronic and printed format to each reviewer with the invitation to evaluate it within 15 days. The reviewers will analyze the paper from several perspectives such as clarity, objectivity of data, scientific quality and relevance. A scale from 1 to 5 is used, where 5 is excellent and 1 is poor. The reviewers score the paper accordingly and issue a recommendation. The existence of a manuscript under review is not revealed to anyone other than peer reviewers and editorial staff. Peer reviewers remain anonymous and are expected to maintain strict confidentiality. After the review process has been completed, authors will be informed by mail of the Editor's decision.

Reviewers should inform the editor about suspected plagiarism. 

Interests and disclosure form", which you can download from the website of Bulletin of Integrative Psychiatry (www.buletindepsihiatrie.ro).

\section{Ethical Considerations}

Authors of articles should sign a statement assuming the responsibility for the originality, property rights and ethical implications of their work. Authors should obtain permission or copy right to publish figures, images or tables from other journals whose material they use.

A written consent must be obtained from patients when personal information or images of patients are included in the article. Authors must state that the protocol has been approved by the Ethics Committee of the institution where the research was conducted.

\section{Address to send the manuscripts is:}

Buletin de Psihiatrie Integrativa/ Institutul de Psihiatrie "Socola" Iasi

Lecturer Alexandra Bolos: Institutul de Psihiatrie "Socola” Iasi, Şos. Bucium 36, 700282, Iasi, jud. Iasi; E-mail: contact@ buletindepsihiatrie.ro 